University of Nebraska - Lincoln

DigitalCommons@University of Nebraska - Lincoln

$10-2010$

\title{
Fragmentation of chloroperoxides: Hypochlorite-mediated dehydration of hydroperoxyacetals to esters
}

Thomas J. Fisher

University of Nebraska-Lincoln, TFisher@unlserve.unl.edu

Patrick Dussault

University of Nebraska-Lincoln, pdussault1@unl.edu

Follow this and additional works at: https://digitalcommons.unl.edu/chemistrydussault

Part of the Chemistry Commons

Fisher, Thomas J. and Dussault, Patrick, "Fragmentation of chloroperoxides: Hypochlorite-mediated dehydration of hydroperoxyacetals to esters" (2010). Patrick Dussault Publications. 15.

https://digitalcommons.unl.edu/chemistrydussault/15

This Article is brought to you for free and open access by the Published Research - Department of Chemistry at DigitalCommons@University of Nebraska - Lincoln. It has been accepted for inclusion in Patrick Dussault Publications by an authorized administrator of DigitalCommons@University of Nebraska - Lincoln. 


\title{
Fragmentation of chloroperoxides: Hypochlorite-mediated dehydration of hydroperoxyacetals to esters
}

\author{
Thomas J. Fisher and Patrick H. Dussault
}

Department of Chemistry, University of Nebraska-Lincoln, Lincoln, NE 68588-0304, USA

Corresponding author - P. H. Dussault, email pdussault1@unl.edu

\begin{abstract}
Hypochlorites efficiently dehydrate hydroperoxyacetals to furnish the corresponding esters. The reaction, which can be accomplished with stoichometric $\mathrm{Ca}(\mathrm{OCl})_{2}$ or with catalytic amounts of $t$-BuOCl, appears to involve formation and heterolytic fragmentation of secondary chloroperoxides, species not previously described in solution chemistry.
\end{abstract}

Keywords: hypochlorite, hydroperoxyacetal, chloroperoxide, fragmentation, ester

\section{Introduction}

Hydroperoxyacetals, readily available intermediates, ${ }^{1}$ are substrates for a number of useful fragmentations, including Fe(II)-mediated cleavage to alkoxy radicals, ${ }^{1(c), 2}$ heterolytic $\mathrm{C}-\mathrm{O}$ bond migrations of tertiary peresters, or persulfonates (Criegee rearrangement), ${ }^{3}$ and the base-promoted dehydration of hydroperoxyacetals, or derived peresters, or persulfonates. ${ }^{1(\mathrm{c})}, 4,5$ In the course of investigations into the addition of oxygen nucleophiles to ozonolysis-derived carbonyl oxides, ${ }^{6}$ we observed the rapid dehydration of secondary hydroperoxyacetals in the presence of commercial bleach. ${ }^{7}$ We now report that $\mathrm{Ca}(\mathrm{OCl})_{2}$, $t$ - $\mathrm{BuOCl}$, and trichloroisocyanuric acid mediate the rapid heterolytic dehydration of hydroperoxyacetals through the apparent intermediacy of secondary chloroperoxides, species whose solution chemistry has not been previously described.

\section{Results and discussion}

Most of the substrates employed in this study were prepared via ozonolysis of alkenes in the presence of an alcohol. ${ }^{\text {1a }}$ Addition of $\mathrm{Ca}(\mathrm{OCl})_{2}$ (1.3 equiv) to $\mathrm{CH}_{3} \mathrm{CN}$ solutions of hydroperoxyacetals $\mathbf{1 a - h}$ furnished esters $\mathbf{2 a - h}$ (Table 1) after evaporation of solvent and filtration through a short silica column. ${ }^{7,8}$ The reaction could also be conducted in $\mathrm{CH}_{3} \mathrm{OH} / \mathrm{CH}_{2} \mathrm{Cl}_{2}$; however the reaction in $\mathrm{CH}_{2} \mathrm{Cl}_{2}$, THF, or toluene was limited by the solubility of $\mathrm{Ca}(\mathrm{OCl})_{2}$. The dehydration proved compatible with a free primary alcohol (entry 4) or a chloroethyl acetal (entry 8). Little reaction was observed with aq $\mathrm{NaOCl}$.

Comparable yields were available with $t$-BuOCl (Table 2). ${ }^{9}$ Reactions, although conducted for the same duration as for $\mathrm{Ca}(\mathrm{OCl})_{2}$, were now complete within $1 \mathrm{~min}$ (TLC). ${ }^{10}$ The rate and yield were not affected by protection from laboratory light (entry 2), use of $\mathrm{CH}_{2} \mathrm{Cl}_{2}$ as solvent (entry 3 ), or the presence of acid (entry 4). However, the presence of methanol slowed reactions considerably (not shown). Dehydration could be conducted with catalytic ( 0.25 equiv) quantities of $t$-BuOCl (entries 5 and 8 ), although the reactions now required $15 \mathrm{~min}$ for completion.

Table 1. Fragmentation of hydroperoxyacetals with $\mathrm{Ca}(\mathrm{OCl})_{2}$

\begin{tabular}{|c|c|c|c|c|c|}
\hline Entry & Substrate & $\mathrm{R}^{1}$ & $\mathrm{R}^{2}$ & Product & Yield $^{\mathrm{a}}$ \\
\hline 1 & $1 a$ & Octyl & $\mathrm{Me}$ & $2 a$ & $75 \%(91)$ \\
\hline 2 & $1 \mathrm{~b}$ & Octyl & Et & $2 b$ & $86 \%(90)$ \\
\hline 3 & $1 c$ & Octyl & $i-\operatorname{Pr}$ & $2 c$ & $83 \%(93)$ \\
\hline 4 & 1d & Octyl & $\left(\mathrm{CH}_{2}\right)_{2} \mathrm{OH}$ & $2 d$ & $83 \%(86)$ \\
\hline 5 & 1e & $\mathrm{AcO}\left(\mathrm{CH}_{2}\right)_{8}$ & $\mathrm{Me}^{2 / 2}$ & $2 e$ & $85 \%(93)$ \\
\hline 6 & 1f & $\mathrm{BnO}\left(\mathrm{CH}_{2}\right)_{3}$ & $\mathrm{Me}$ & $2 f$ & $81 \%(94)$ \\
\hline 7 & $1 \mathrm{~g}$ & $\mathrm{Ph}\left(\mathrm{CH}_{2}\right)_{2}$ & $\mathrm{Me}$ & $2 g$ & $80 \%(93)$ \\
\hline 8 & $1 \mathrm{~h}$ & Octyl & $\left(\mathrm{CH}_{2}\right)_{2} \mathrm{Cl}$ & $2 h$ & $82 \%(92)$ \\
\hline
\end{tabular}

a. Isolated yields on $0.5 \mathrm{mmol}$ or $(5 \mathrm{mmol})$ scale. 
Table 2. Fragmentation of hydroperoxyacetals with $t$-BuOCl $t$-BuOCl

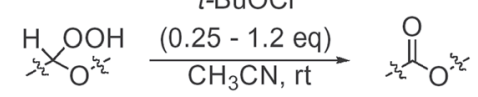

\begin{tabular}{llllll}
\hline Entry & Substrate & $t$-BuOCl (equiv) & $t(\mathrm{~min})$ & Product & Yield $^{\mathbf{a}}(\%)$ \\
\hline 1 & $\mathbf{1 a}$ & 1.2 & 10 & $\mathbf{2 a}$ & 77 \\
$2^{\mathrm{b}}$ & $\mathbf{1 a}$ & 1.2 & 10 & $\mathbf{2 a}$ & 77 \\
$3^{\mathrm{c}}$ & $\mathbf{1 a}$ & 1.2 & 10 & $\mathbf{2 a}$ & 79 \\
$4^{\mathrm{d}}$ & $\mathbf{1 a}$ & 1.2 & 10 & $\mathbf{2 a}$ & 84 \\
5 & $\mathbf{1 a}$ & 0.25 & 15 & $\mathbf{2 a}$ & 78 \\
6 & $\mathbf{1 b}$ & 1.2 & 10 & $\mathbf{2 b}$ & 85 \\
7 & $\mathbf{1 d}$ & 1.2 & 10 & $\mathbf{2 d}$ & 84 \\
8 & $\mathbf{1 e}$ & 0.25 & 15 & $\mathbf{2 e}$ & 85 \\
9 & $\mathbf{1 h}$ & 1.2 & 10 & $\mathbf{2 h}$ & 84 \\
\hline
\end{tabular}

a. Isolated yields on $0.5 \mathrm{mmol}$ scale.

b. Protected from light (Al foil over flask).

c. $\mathrm{CH}_{2} \mathrm{Cl}_{2}$ as solvent.

d. Added HOAc ( $\geq 2$ equiv).

No reaction was observed between $t$ - $\mathrm{BuOCl}$ and a silylated hydroperoxyacetal (1i, Scheme 1). However, a secondary hydroperoxide $(\mathbf{1 j})^{11}$ underwent rapid dehydration; the low yield likely reflects product volatility. Dehydration of secondary allylic hydroperoxides (not shown) required excess $t$ - $\mathrm{BuOCl}$ and furnished the expected $\mathrm{a}, \beta-$ unsaturated ketones as mixtures with significant amounts of byproducts lacking unsaturation.

The fragmentation can be combined with alkene ozonolysis to afford a convenient one-pot synthesis of esters (Scheme 2).

The fragmentation is likely to involve the initial formation of chloroperoxides, species previously prepared only in tertiary systems. ${ }^{12}$ The intermediacy of $\mathrm{ROOCl}$ is consistent with the lack of reaction of silylated hydroperoxyacetal 1i. In an effort to access chloroperoxides with reagents other than hypochlorites, we discovered that commercially available trichloroisocyanuric acid promotes the dehydrative fragmentation as or more efficiently than $t$ - $\mathrm{BuOCl}$ (Scheme 3).

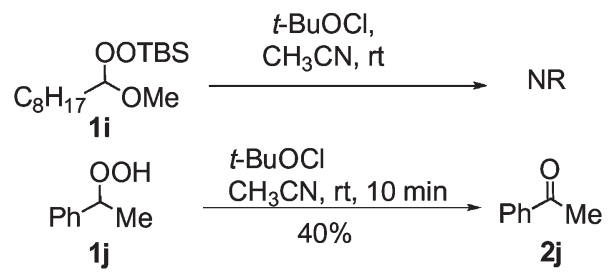

Scheme 1. Substrates other than hydroperoxyacetals.

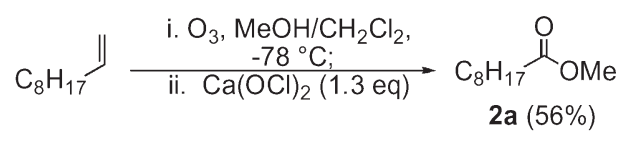

Scheme 2. Application in tandem with ozonolysis.

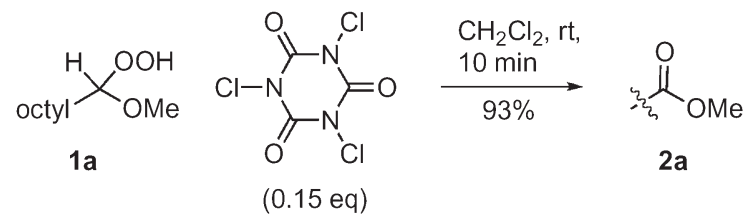

Scheme 3. Dehydration with trichloroisocyanuric acid.
The conversion of the chloroperoxides to esters could in principle proceed through either homolytic or heterolytic pathways (Scheme 4), and several additional experiments were conducted to discriminate between these possibilities (Scheme 5).

Chloroperoxides are reported to undergo homolytic scission to generate ROO* and $\mathrm{Cl}^{*} .{ }^{12,13}$ For this reason, we initially hypothesized the dehydrations involved a radical chain initiated by abstraction of the acetal $\mathrm{C}-\mathrm{H}$; the resulting carbon radical would be expected to fragment to the product ester and a propagating radical ("OCl). However, the dehydrations were insensitive to the presence or the absence of visible light and did not occur in the presence of $\mathrm{PhI}(\mathrm{OTFA})_{2}$, a reagent known to promote peroxyl radical formation. ${ }^{14}$ Perhaps most convincingly, dehydration of $\mathbf{1 k}$, a hydroperoxyacetal substrate incorporating a fast radical clock (Scheme 5), proceeded with no detectable formation of ring-opened products. ${ }^{15}$

The potential role of alkoxy radicals was probed with hydroperoxyacetals $\mathbf{1 1}$ and $\mathbf{1} \mathbf{m}^{16}$ (Scheme 5); the a-oxygenated alkoxy radicals derived from either substrate would be expected to readily undergo $\beta$-scission. ${ }^{17}$ However, both 11 and 1m undergo dehydration with no signs of radical cleavage. In contrast, a hydroperoxyketal unable to dehydrate (1n) undergoes a much slower (30 $\mathrm{min}$ ) reaction to furnish chloroalkanoate 3 , the product of alkoxy radical cleavage.

The results support fragmentation through the heterolytic pathway illustrated in Scheme 4, presumably through a mechanism analogous to Criegee or Hock fragmentation (activation of hydroperoxides by protonation or Lewis acid
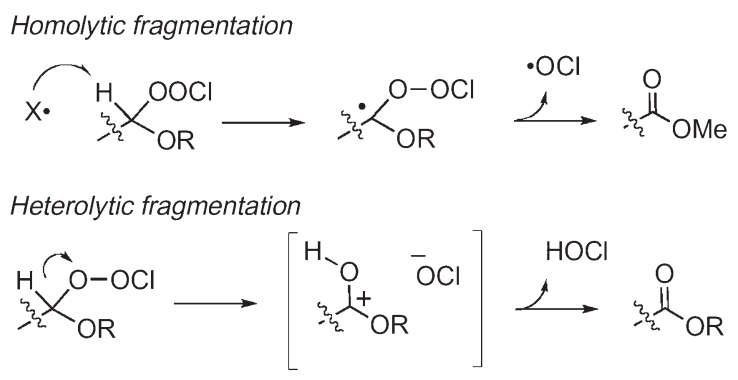

Scheme 4. Mechanistic possibilities.

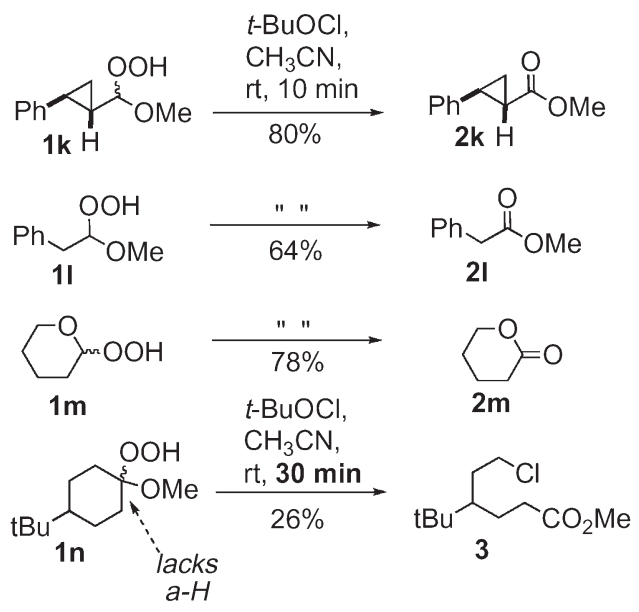

Scheme 5. Additional substrates. 
complexation). ${ }^{3,18}$ The specificity for migration of hydrogen relative to $\mathrm{Ph}$ or $\mathrm{Bn}(\mathbf{1 j}, \mathbf{1 1})$ under nonbasic conditions is interesting. However, as has recently been demonstrated for Baeyer-Villiger rearrangements of alkoxybromanes derived from hemiacetals, ${ }^{19}$ the nature of the activating reagents can have a strong influence on rearrangements to electron-deficient oxygen. The proposed mechanism predicts the regeneration of $\mathrm{HOCl}_{1}{ }^{20}$ and is consistent with the high conversion obtained in the presence of substoichiometric $t$ - $\mathrm{BuOCl}$ or trichloroisocyanuric acid. The results may be of relevance to atmospheric decomposition of primary chloroperoxides. ${ }^{21}$

\section{Conclusions}

We have developed a new fragmentation of hydroperoxyacetals to esters based upon heterolytic fragmentation of intermediate chloroperoxides.

- CAUtion: While we experienced no hazards in the course of this work, any preparative work with peroxides should be conducted with an awareness of the potential for spontaneous and exothermic decomposition reactions. ${ }^{22}$

Acknowledgments - This research was funded by NSF (CH0749916) and the Nebraska Research Initiative and conducted in facilities remodeled with support from NIH (RR016544-01). NMR spectra were acquired, in part, on spectrometers purchased with NSF support (MRI 0079750 and CHE 0091975). We thank Dr. Chris Schwartz and Professor Stephen DiMagno for helpful discussions.

\section{Notes and References}

1. (a) K. Zmitek, M. Zupan and J. Iskra, Org. Biomol. Chem., 5 (2007), p. 3895 (a-oxygenated hydroperoxides, transacetalization). (b) A. Scarso and G. Strukul, A. Berkessel, Editor, Science of Synthesis, vol. 38 (Stuggart: Thieme, 2009), pp. 36-52 (trapping of carbonyl oxides). (c) P. H. Dussault in Active Oxygen in Chemistry, C. S. Foote, J. S. Valentine, A. Greenberg, \& J. F. Liebman, editors (London: Blackie A\&P, 1995), pp. 141-203 (addition to enol ethers). (d) P. H. Dussault, I. Q. Lee, H. J. Lee, R. J. Lee, Q. J. Niu, J. A. Schultz, and U. Zope, J. Org. Chem., 65 (2000), p. 8407 (photooxygenation of enol ethers).

2. S. K. Ivanov in Methoden der Organischen Chemie, H. Kropf, editor, 4th ed., vol. E13 (Stuttgart: Thieme, 1988), pp. 1031-1037.

3. R. Criegee. Liebigs Ann., 560 (1948), p. 127; H. Kropf in Methoden der Organischen Chemie, H. Kropf, editor, 4th ed., vol. E13 (Stuttgart: Thieme, 1988), pp. 1095-1099.

4. (a) R. M. Ellam and J. M. Padbury. J. Chem. Soc., Chem. Commun. (1972), p. 1086; Base-promoted fragmentation of hydroperoxyacetals is likely involved in a direct ozonolytic conversion of terminal alkenes to methyl esters. (b) J. A. Marshall and A. W. Garofalo. J. Org. Chem., 58 (1993), p. 3675; G. Evano in Science of Synthesis, vol. 20b, J. S. Panek, editor (Stuttgart: Thieme, 2007), pp. 795-797.

5. Q. E. Thompson. J. Org. Chem., 27 (1962), p. 4498; R. E. Claus and S. L. Schreiber. Org. Synth., 64 (1986), p. 150.
6. C. Schwartz, J. Raible, K. Mott, and P. H. Dussault. Tetrahedron, 62 (2006), p. 10747.

7. 2.0 weight equiv of commercial solid bleach ( $\sim 65 \%$ purity) delivers $1.3 \mathrm{~mol}$ equiv of $\mathrm{Ca}(\mathrm{OCl})_{2}$; other components include $\mathrm{NaCl}, \mathrm{CaCl}_{2}, \mathrm{Ca}\left(\mathrm{ClO}_{3}\right)_{2}$, and water.

8. Typical procedure for reactions with $\mathrm{Ca}(\mathrm{OCl})_{2}$ : To a flame-dried $8 \mathrm{~mL}$ vial equipped with stirbar and screw-top septa cap containing technical grade $\mathrm{Ca}(\mathrm{OCl})_{2}(1.3$ equiv, $1.0 \mathrm{mmol}, 143 \mathrm{mg}$ of $65 \%$ reagent) is added $\mathrm{CH}_{3} \mathrm{CN}(2 \mathrm{~mL})$. A solution of the hydroperoxy acetal (1.0 equiv, $0.50 \mathrm{mmol})$ in $\mathrm{CH}_{3} \mathrm{CN}(1 \mathrm{ml})$ is added as a single portion via syringe. The reaction is stirred vigorously for $10 \mathrm{~min}$ at which time the partially heterogeneous solution is filtered through a short plug of silica. The reaction vial is rinsed with a small amount of $\mathrm{CH}_{2} \mathrm{Cl}_{2}$ and this solution is also filtered through the silica plug. The silica plug is rinsed with another small portion of $\mathrm{CH}_{2} \mathrm{Cl}_{2}$ and the filtrate is concentrated.

9. M. J. Mintz and C. Walling. Org. Synth., 49 (1969), p. 9.

10. Typical procedure for reactions with $t-\mathrm{BuOCl}$ : To a flame dried $8 \mathrm{~mL}$ vial equipped with stirbar and screw-top septa cap containing $t$ - $\mathrm{BuOCl}$ (1.2 equiv, $0.6 \mathrm{mmol}, 65 \mathrm{mg}$ ) is added $\mathrm{CH}_{3} \mathrm{CN}$ $(2 \mathrm{~mL})$. The hydroperoxy acetal (1 equiv, $0.5 \mathrm{mmol})$ is dissolved in $\mathrm{CH}_{3} \mathrm{CN}(1 \mathrm{~mL})$ and added in one portion via syringe. The reaction is stirred vigorously for $10 \mathrm{~min}$ and then worked up as before.

11. Prepared by Ag(I)-mediated displacement: P. G. Cookson, A. G. Davies, and B. P. Roberts, J. Chem. Soc., Chem. Commun., (1976), p. 1022.

12. A. N. Osipov, O. M. Panasenko, A. V. Chekanov, and J. Arnhold. Free Radical Res., 36 (2002), p. 749.

13. P. Marwah, A. Marwah, and H. A. Lardy. Green Chem., 6 (2004), p. 570.

14. M. Ochiai, T. Ito, H. Takahashi, A. Nakanishi, M. Toyonari, T. Suleda, S. Goto, and M. Shiro. J. Am. Chem. Soc., 118 (1996), p. 7716; N. A. Milas and B. Plesnicar. J. Am. Chem. Soc., 90 (1968), p. 4450.

15. $\mathrm{k}_{\text {opening }} \geq 10^{11} / \mathrm{s}$ for the unsubstituted 2-phenylcyclopropyl methyl radical: M. H. L. TadicBiadatti and M. Newcomb, J. Chem. Soc., Perkin Trans. 2, (1996), p. 1467.

16. $1 \mathrm{~m}$ was prepared via addition of $\mathrm{H}_{2} \mathrm{O}_{2}$ to dihydropyran: $\mathrm{N}$. A. Milas, R. L. Peeler, and O. L. Mageli, J. Am. Chem. Soc., 76 (1954), p. 2322.

17. $\beta$-Scission of oxygenated alkoxy radicals: J. J. Orlando, G. S. Tyndall, and T. J. Wallington, Chem. Rev., 103 (2003), p. 4657; S. Erhardt, S. A. Macgregor, K. J. McCullough, K. Savill, and B. J. Taylor. Org. Lett., 9 (2007), p. 5569.

18. H. Hock and H. Kropf. Angew. Chem., 69 (1957), p. 313; P. H. Dussault, H. J. Lee, and X. Liu. Perkin. 1, (2000), p. 3006.

19. M. Ochiai, A. Yoshimura, K. Miyamoto, S. Hayashi, and W. Nakanishi. J. Am. Chem. Soc., 132 (2010), p. 9236.

20. Chlorine test strips revealed the diluted aqueous layer from stoichometric reactions to have up to $50-60 \%$ of the theoretical amount of active chlorine.

21. M. Schnell, M. Mühlhäuser, and S. D. Peyerimhoff. J. Phys. Chem. A, 108 (2004), p. 1298.

22. L. A. Medard in Accidental Explosions: Types of Explosive Substances, vol. 2 (Chichester: Ellis Horwood Ltd, 1989); P. A. Patnaik, Comprehensive Guide to the Hazardous Properties of Chemical Substances, 3rd ed. (Hoboken, NJ: John Wiley and Sons, 2007); J. Zabicky in The Chemistry of the Peroxide Group, Z. Rappoport, editor (Chichester: John Wiley and Sons, 2006), vol. 2, pp. 597-773, pt 2.

Supplementary data $-{ }^{1} \mathrm{H}$ and ${ }^{13} \mathrm{C}$ NMR spectra for $\mathbf{1 a}-\mathbf{i}, \mathbf{1} \mathbf{k}-\mathbf{n}, \mathbf{2} \mathbf{a}-\mathbf{I}, \mathbf{2 k}-\mathbf{m}, \& \mathbf{3}$ are presented in the following pages. 
Supporting Information

"Fragmentation of chloroperoxides: hypochlorite-mediated dehydration of hydroperoxyacetals to esters"

Fisher, T. J. and Dussault, Patrick H.

${ }^{1} \mathrm{H}$ NMR and ${ }^{13} \mathrm{C}$ NMR spectra

1-Methoxynonyl hydroperoxide 1H (1a)

1-Methoxynonyl hydroperoxide 13C (1a)

1-Ethoxynonyl hydroperoxide $1 \mathrm{H} \quad(\mathbf{1 b})$

1-Ethoxynonyl hydroperoxide 13C (1b)

1-Isopropoxynonyl hydroperoxide $1 \mathrm{H} \quad(\mathbf{1 c})$

1-Isopropoxynonyl hydroperoxide 13C (1c)

1-(2-Hydroxyethoxy)nonyl hydroperoxide $1 \mathrm{H} \quad$ (1d)

1-(2-Hydroxyethoxy)nonyl hydroperoxide 13C (1d) 10

9-Hydroperoxy-9-methoxynonyl acetate $1 \mathrm{H} \mathrm{(1e)} 11$

9-Hydroperoxy-9-methoxynonyl acetate 13C NMR (1e) 12

4-Benzyloxy-1-methoxybutyl hydroperoxide 1H NMR (1f) 13

4-Benzyloxy-1-methoxybutyl hydroperoxide 13C (1f) 14

1-Methoxy-3-phenylpropyl hydroperoxide 1H NMR (1g) 15

1-Methoxy-3-phenylpropyl hydroperoxide 13C NMR (1g) 16

1-(2-Chloroethoxy)nonyl hydroperoxide 1H (1h) 17

1-(2-Chloroethoxy)nonyl hydroperoxide 13C (1h) 18

1,1-Dimethyl-tert-butyl-(1'-methoxynonyldioxy)silane 1H (1i) 19

1,1-Dimethyl-tert-butyl-(1'-methoxynonyldioxy)silane 13C (1i) 20

trans-2-(2-Phenylcyclopropyl)-1-methoxyethyl hydroperoxide 1H (1k) 21

trans-2-(2-Phenylcyclopropyl)-1-methoxyethyl hydroperoxide 13C (1k) 22

1-Methoxy-2-phenylethyl hydroperoxide 1H (11) 23

1-Methoxy-2-phenylethyl hydroperoxide 13C (11) 24

2-Tetrahydropyranyl hydroperoxide $1 \mathrm{H} \mathrm{(1 \mathbf {m } )} 25$

2-Tetrahydropyranyl hydroperoxide 13C (1 m) 26

4-tert-Butyl-1-methoxycyclohexyl hydroperoxide 1H (1n) 27

4-tert-Butyl-1-methoxycyclohexyl hydroperoxide 13C (1n) 28

$\begin{array}{ll}\text { Methyl nonanoate 1H (2a) } & 29\end{array}$

Methyl nonanoate 13C (2a) 30

Ethyl nonanoate $1 \mathrm{H}(\mathbf{2 b}) \quad 31$

Ethyl nonanoate 13C (2b) $\quad 32$

Isopropyl nonanoate $1 \mathrm{H} \quad \mathbf{( 2 c )} \quad 33$

Isopropyl nonanoate 13C (2c) $\quad 34$

2-Hydroxyethyl nonanoate 1H (2d) 35

2-Hydroxyethyl nonanoate 13C (2d) 36

Methyl 9-acetoxy nonanoate 1H (2e) 37

Methyl 9-acetoxy nonanoate 13C (2e) 38

Methyl 4-benzyloxy butyrate 1H (2f) 39

Methyl 4-benzyloxy butyrate 13C (2f) 40

Methyl 3-phenyl propionate 1H (2g) 41 
Methyl 3-phenyl propionate 13C (2g) 42

2-Chloroethyl nonanoate 1H (2h) 43

2-Chloroethyl nonanoate 13C (2h) 44

trans-Methyl 2-phenylcyclopropanecarboxylate 1H (2k) 45

trans-Methyl 2-phenylcyclopropanecarboxylate 13C (2k) 46

Methyl benzoate 1H (2l) $\quad 47$

Methyl benzoate 13C (2l) 48

$\delta$-Valerolactone $1 \mathrm{H} \quad(\mathbf{2 m}) \quad 49$

$\delta$-Valerolactone 13C (2m) 50

Methyl 4-tert-Butyl-6-chloro hexanoate 1H (3) 51

Methyl 4-tert-Butyl-6-chloro hexanoate 13C (3) 52 


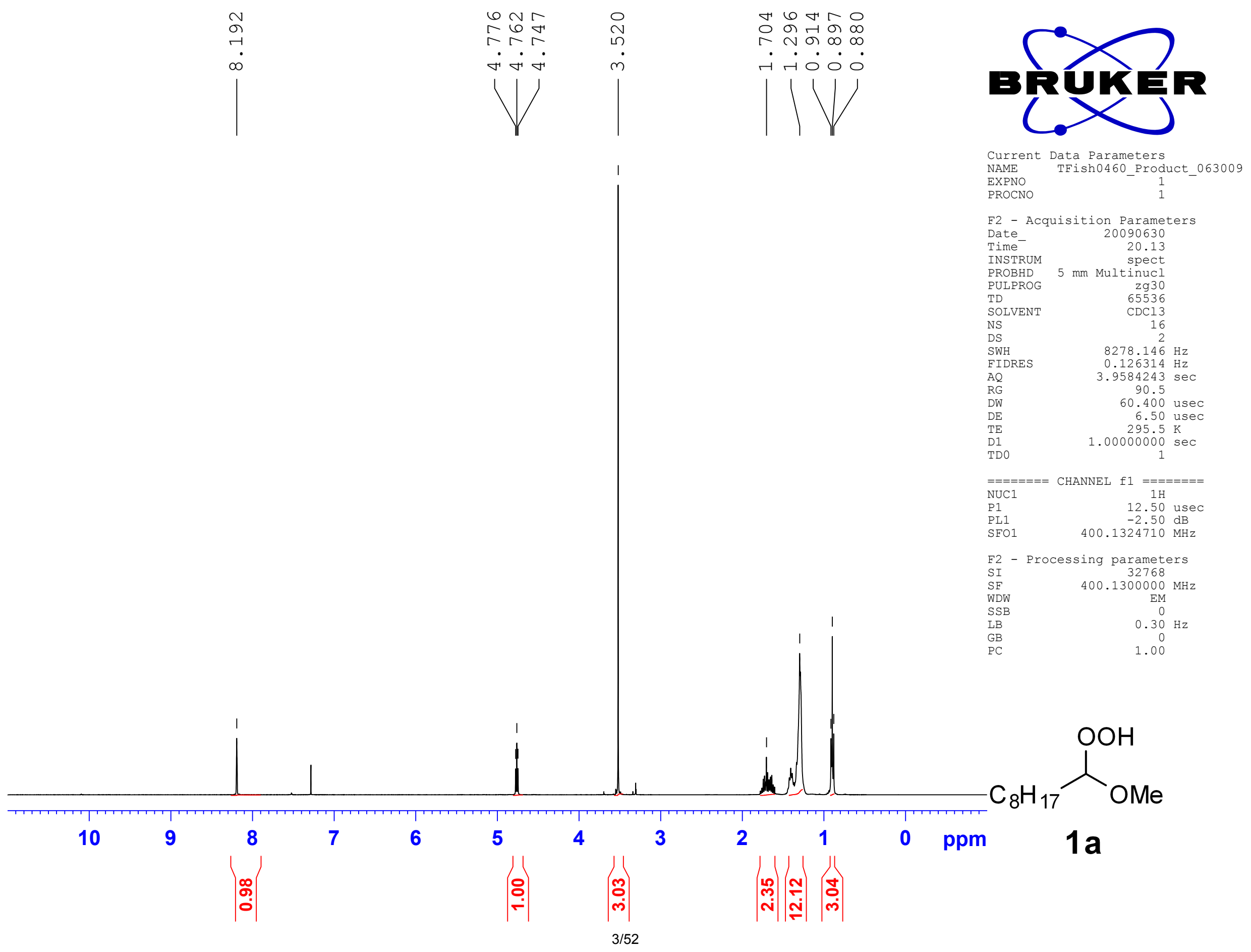




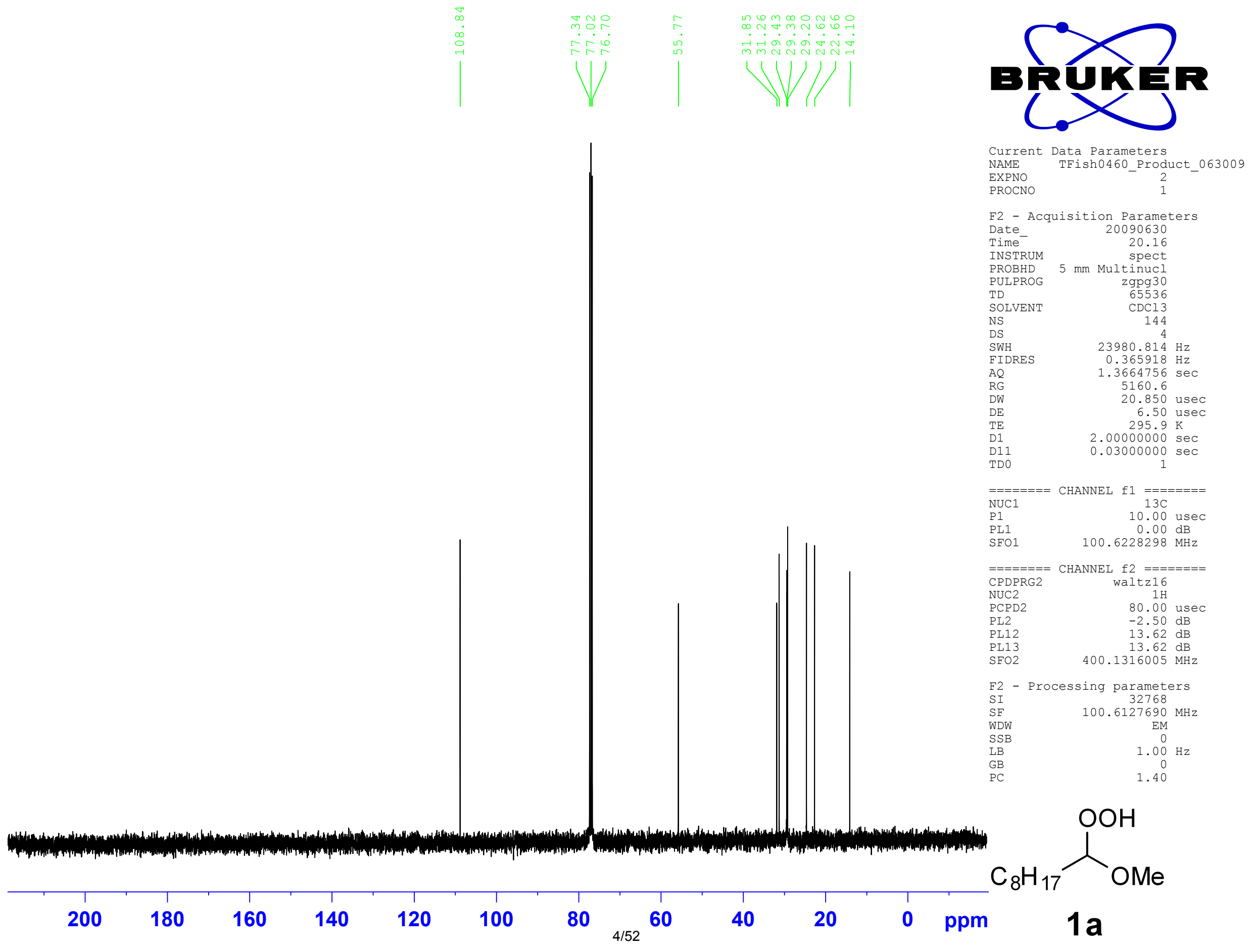




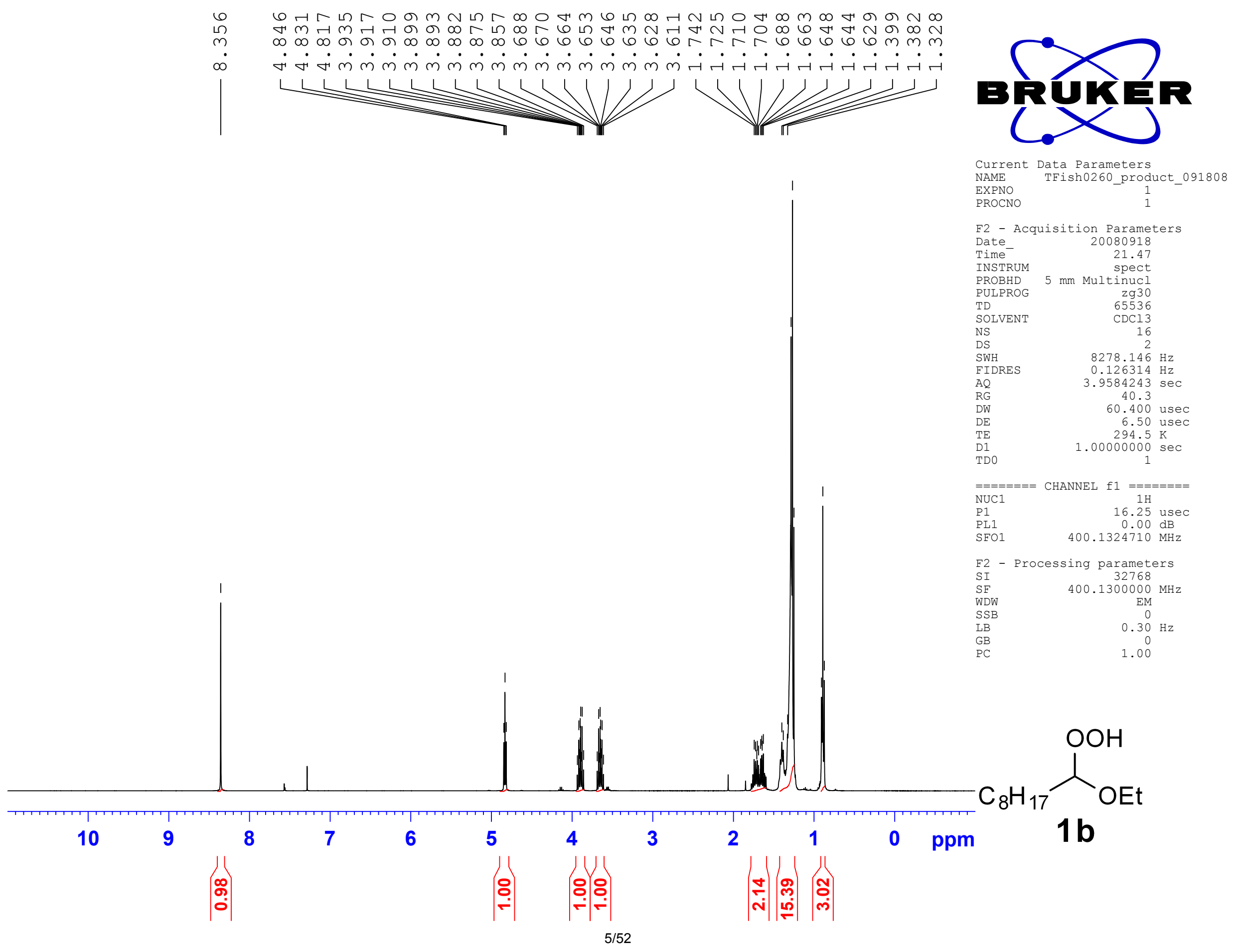




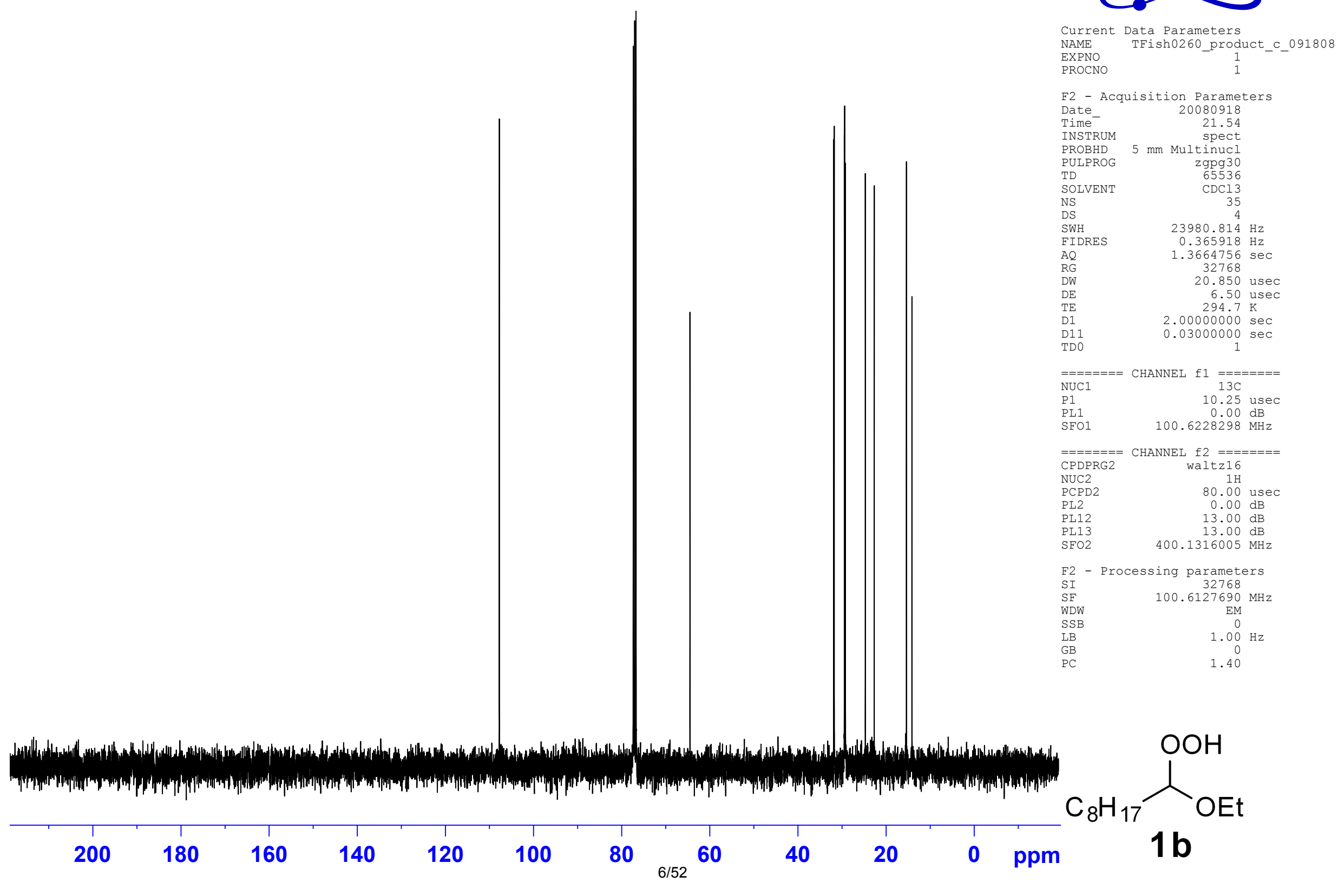




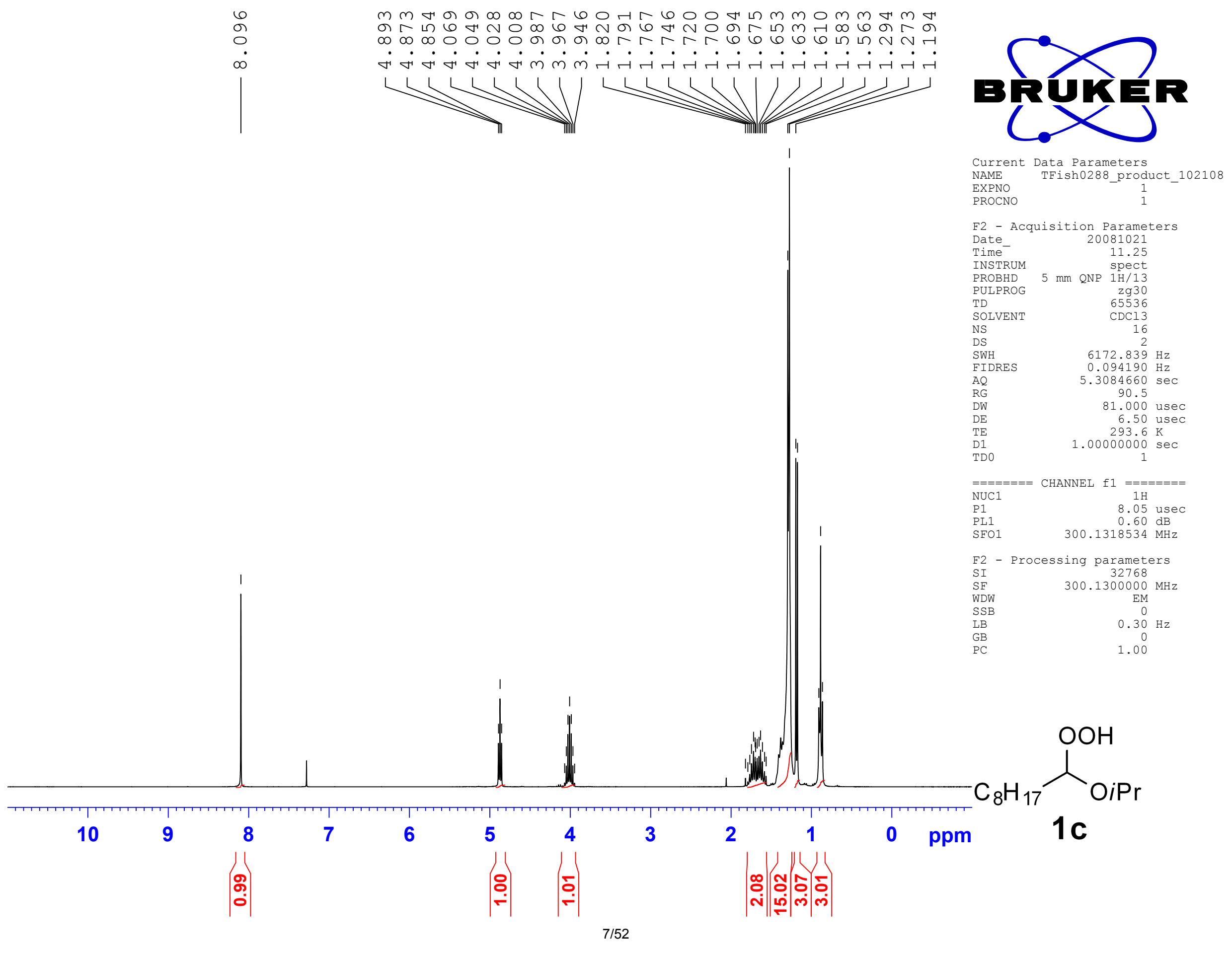




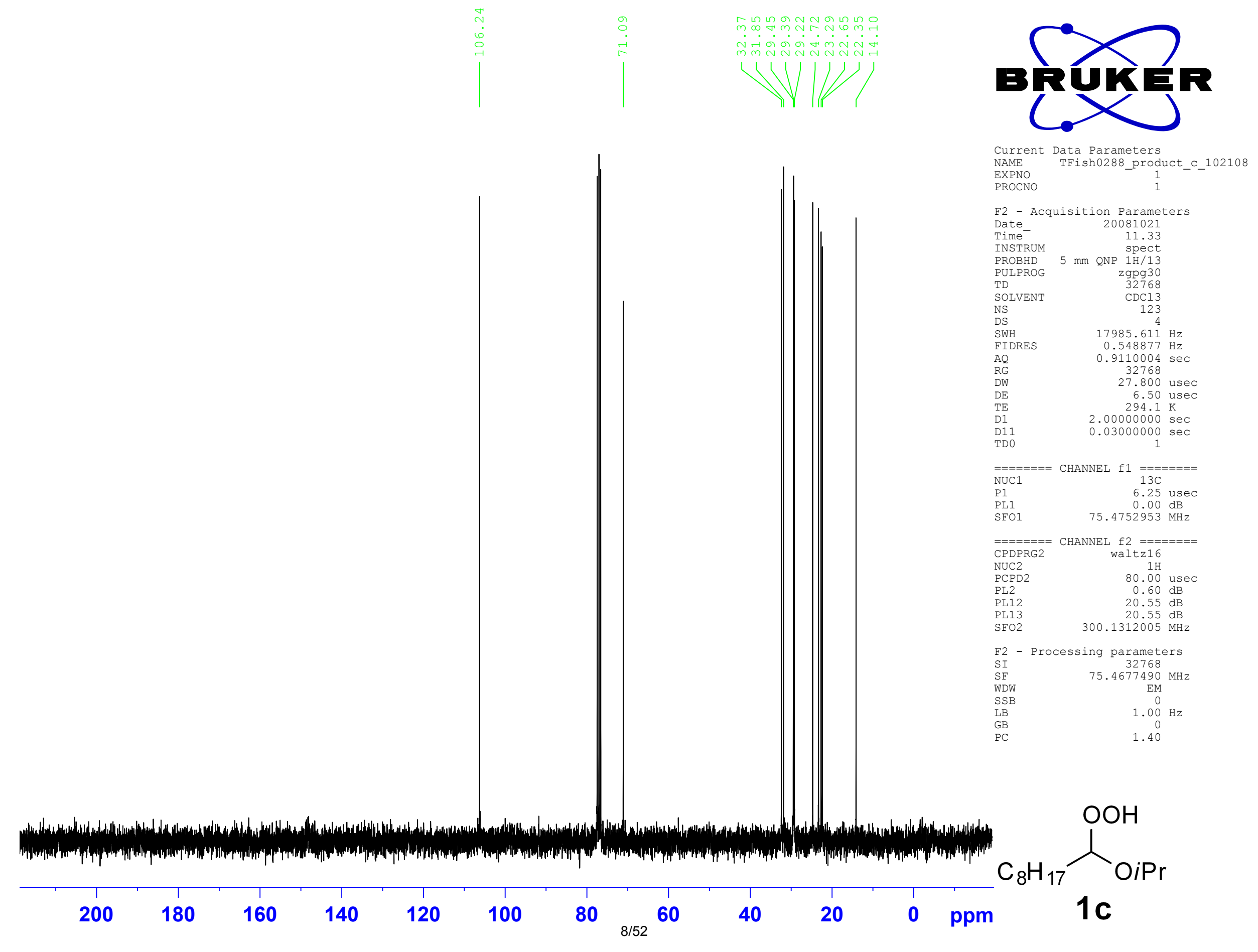




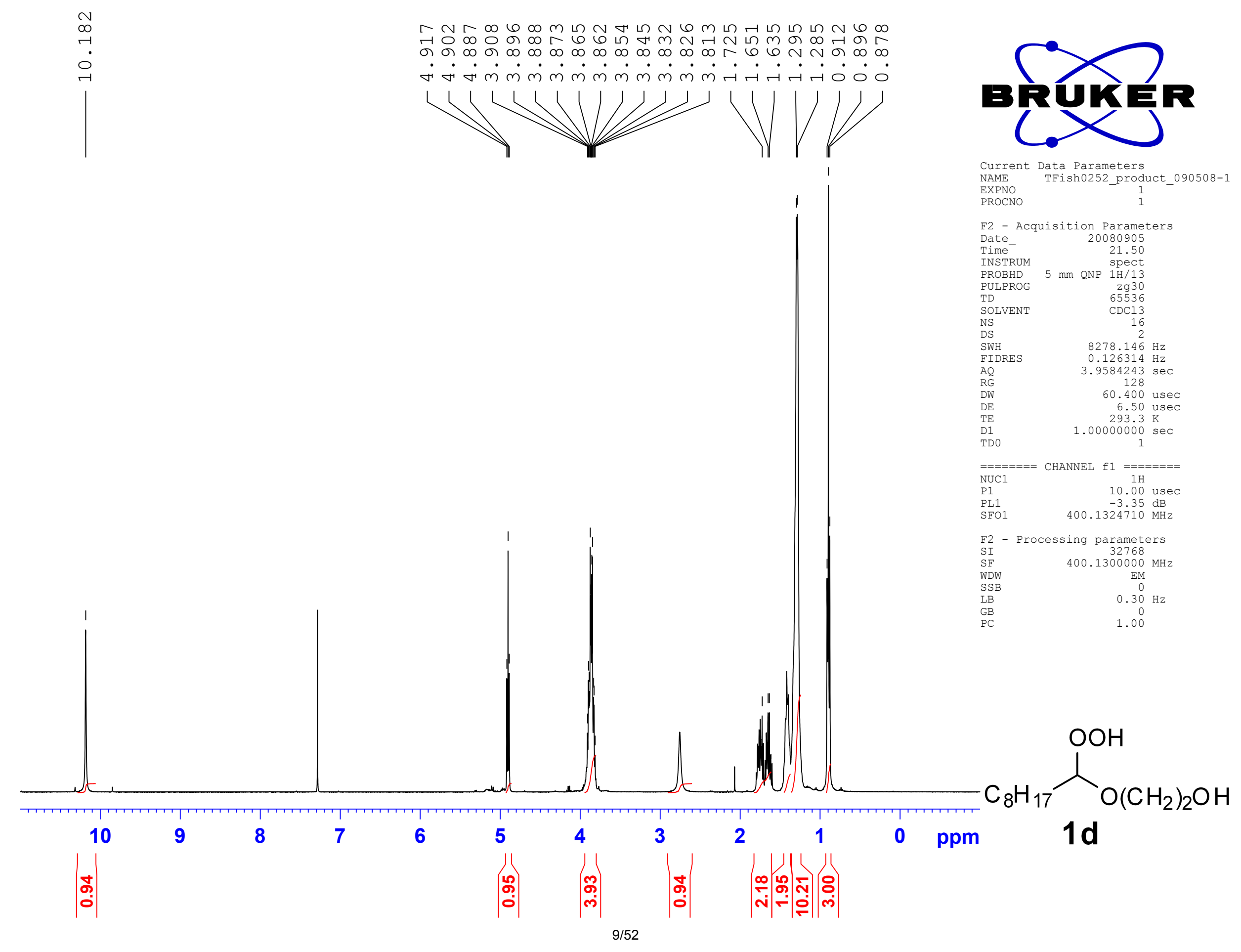




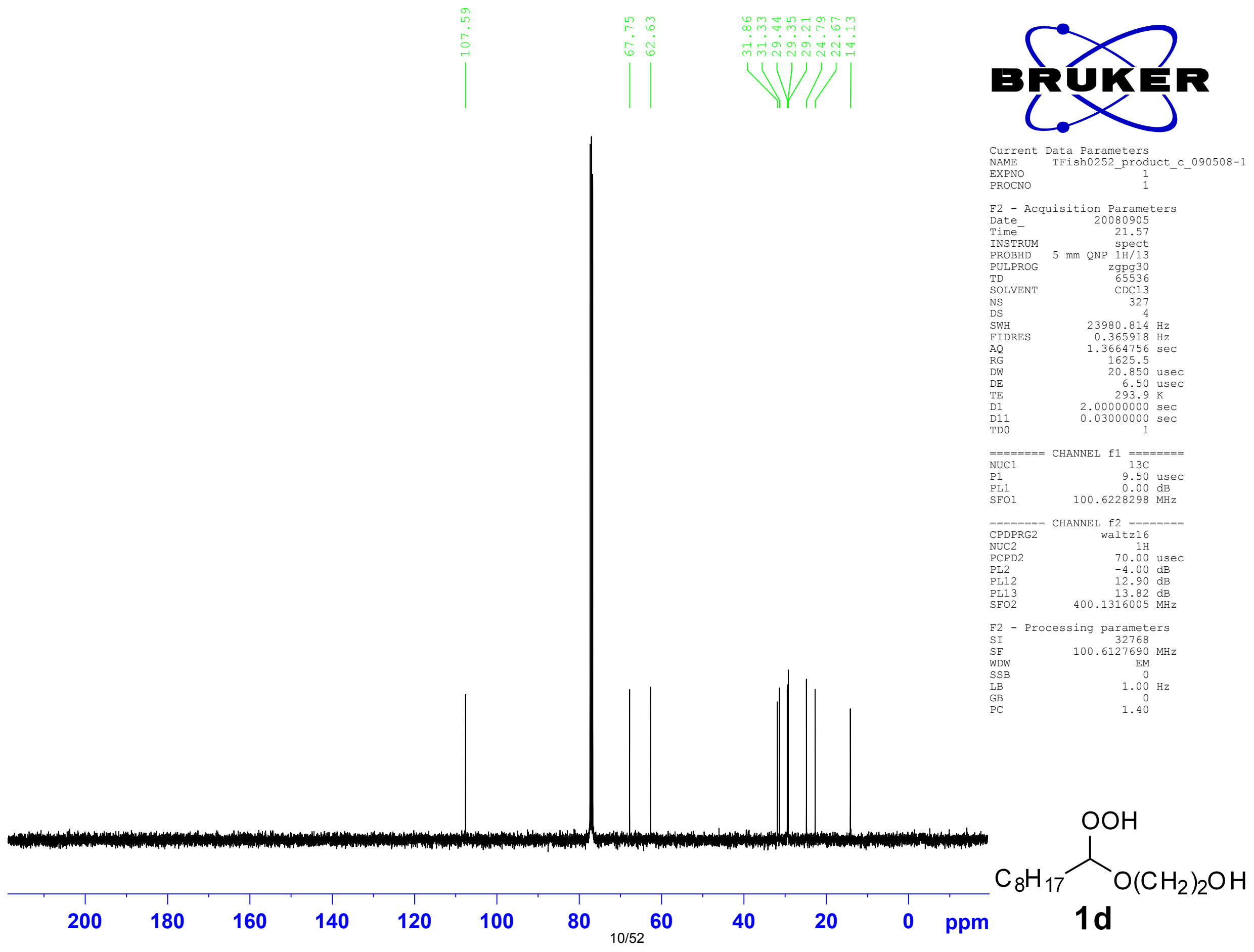




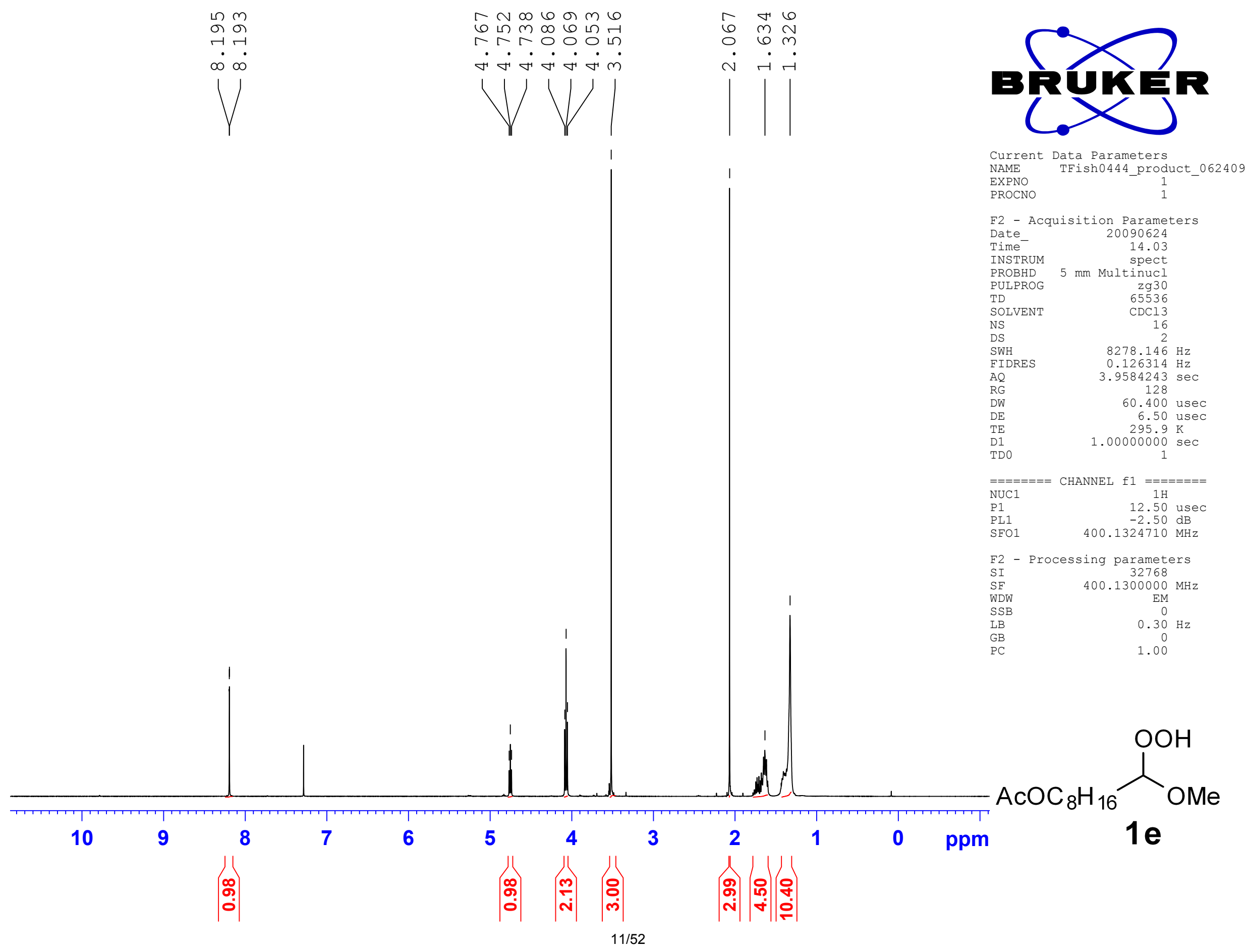




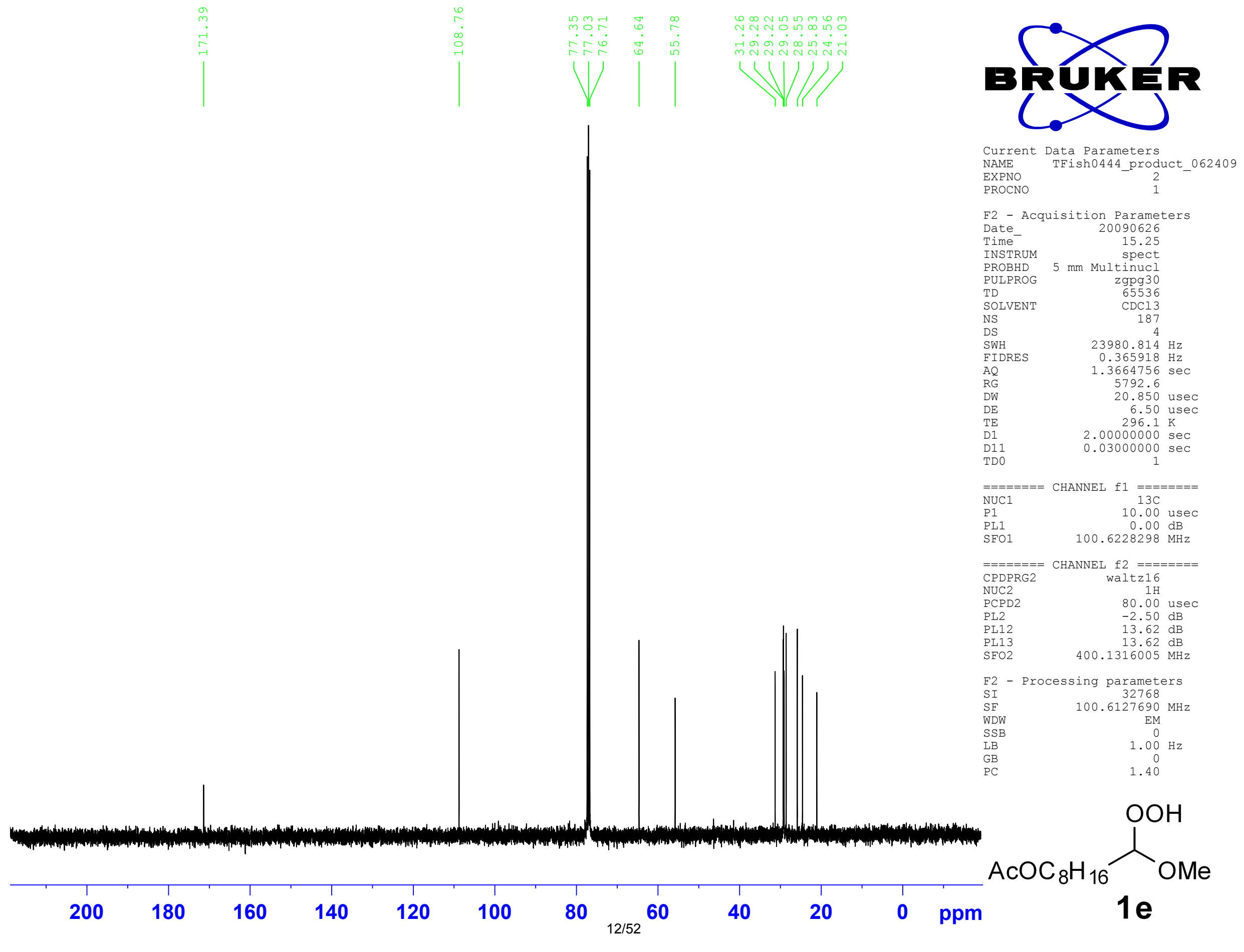




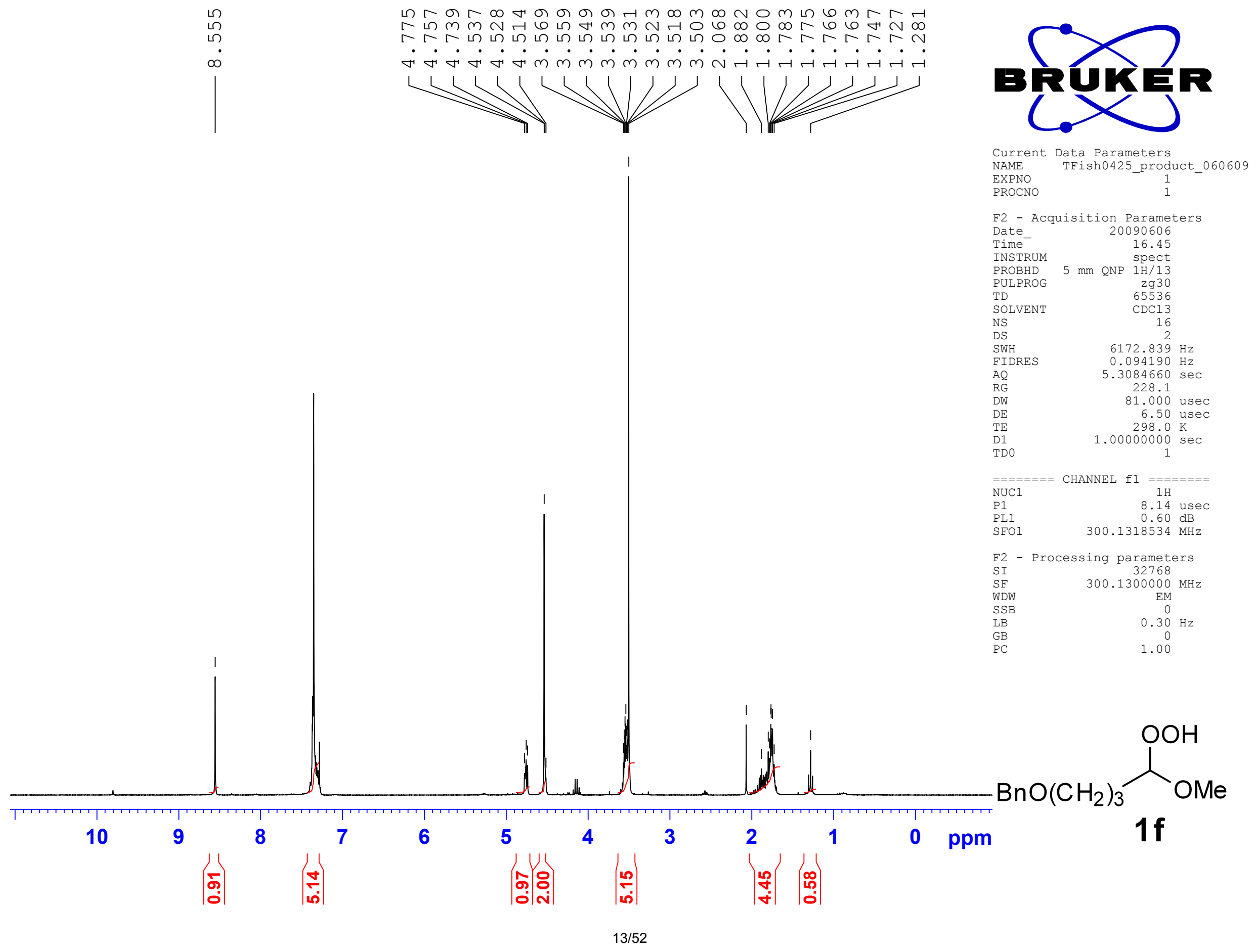




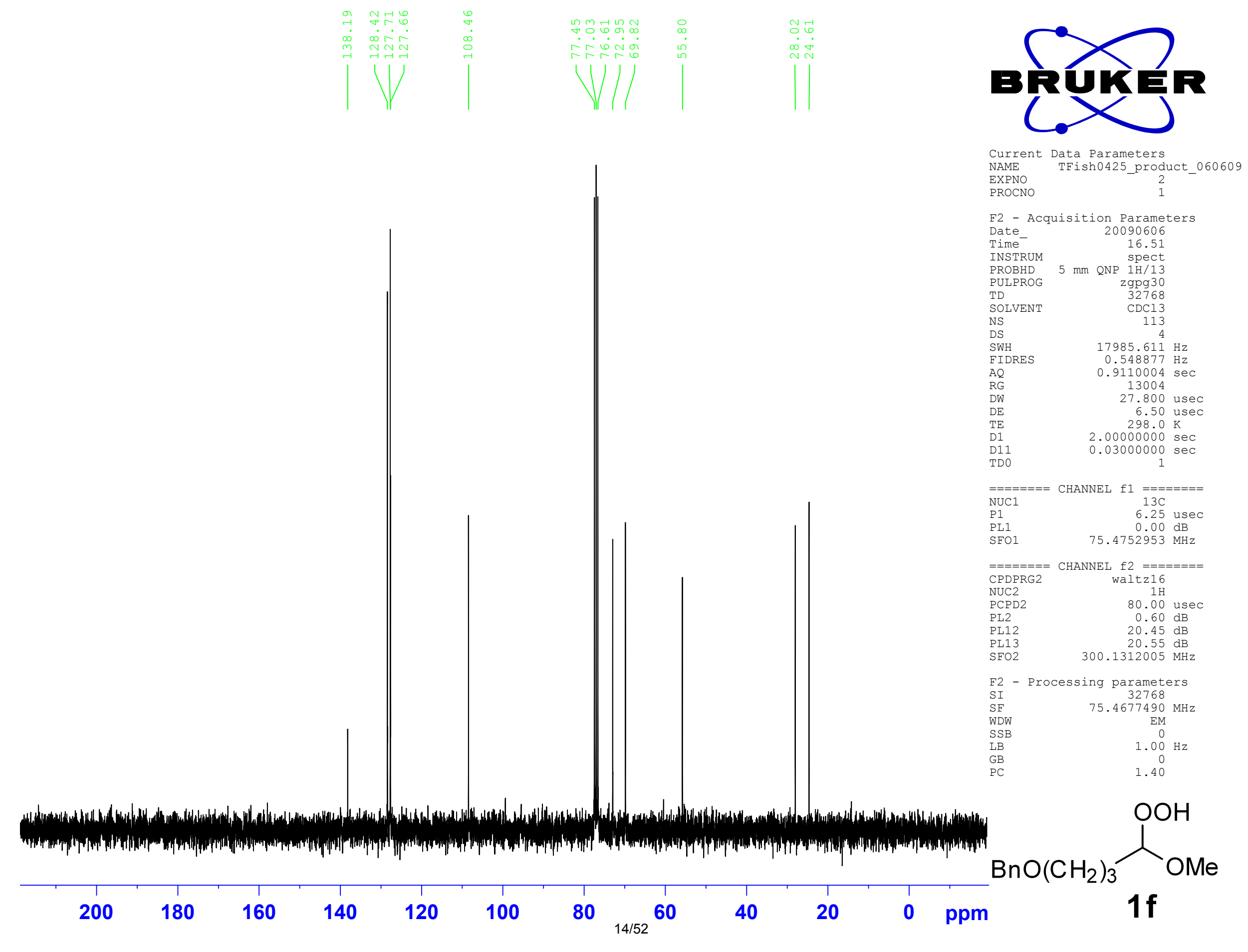




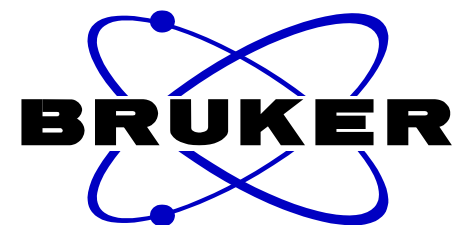

current Data Parameters NAME TFish0452 produc EXPNO

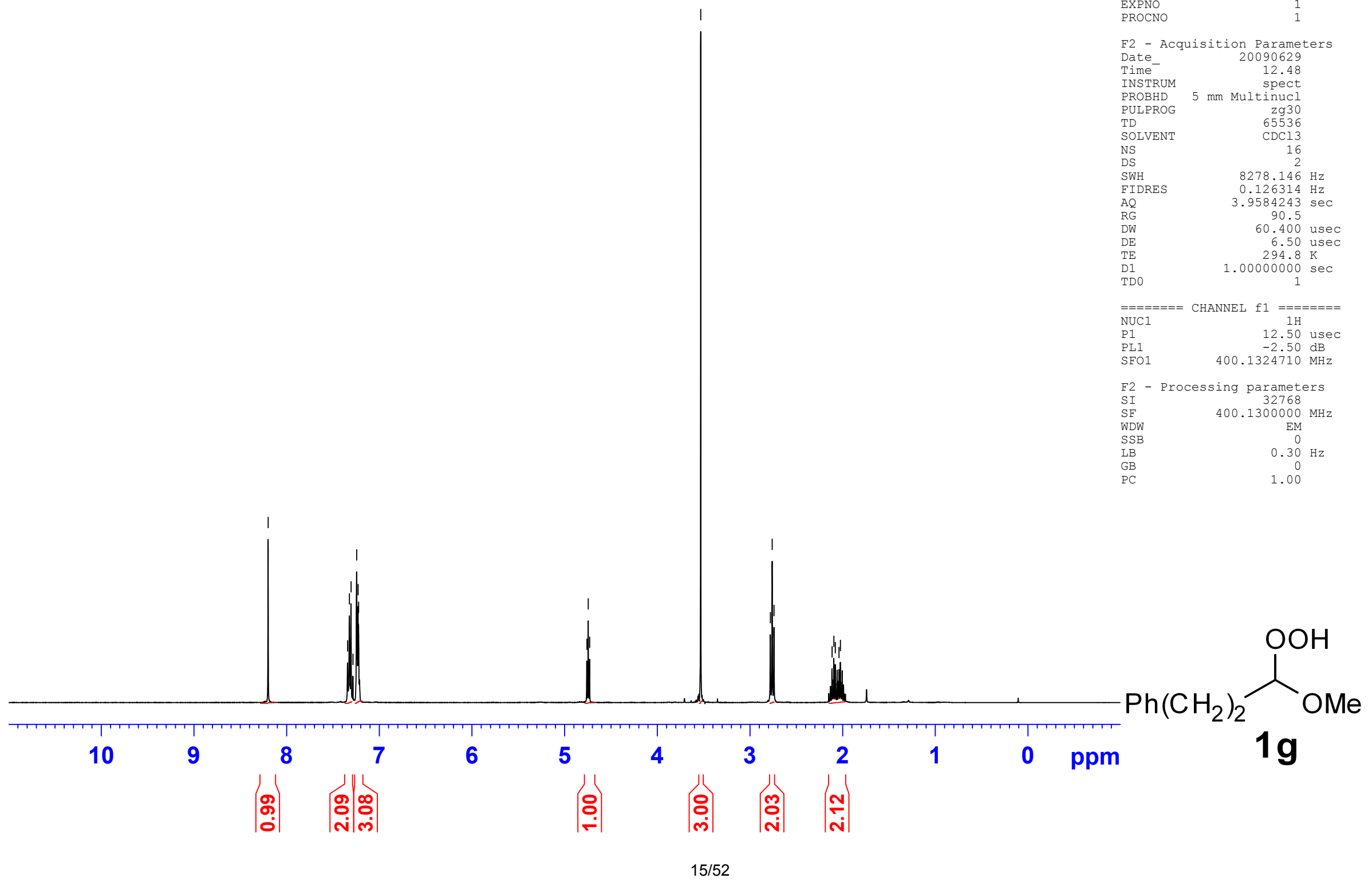




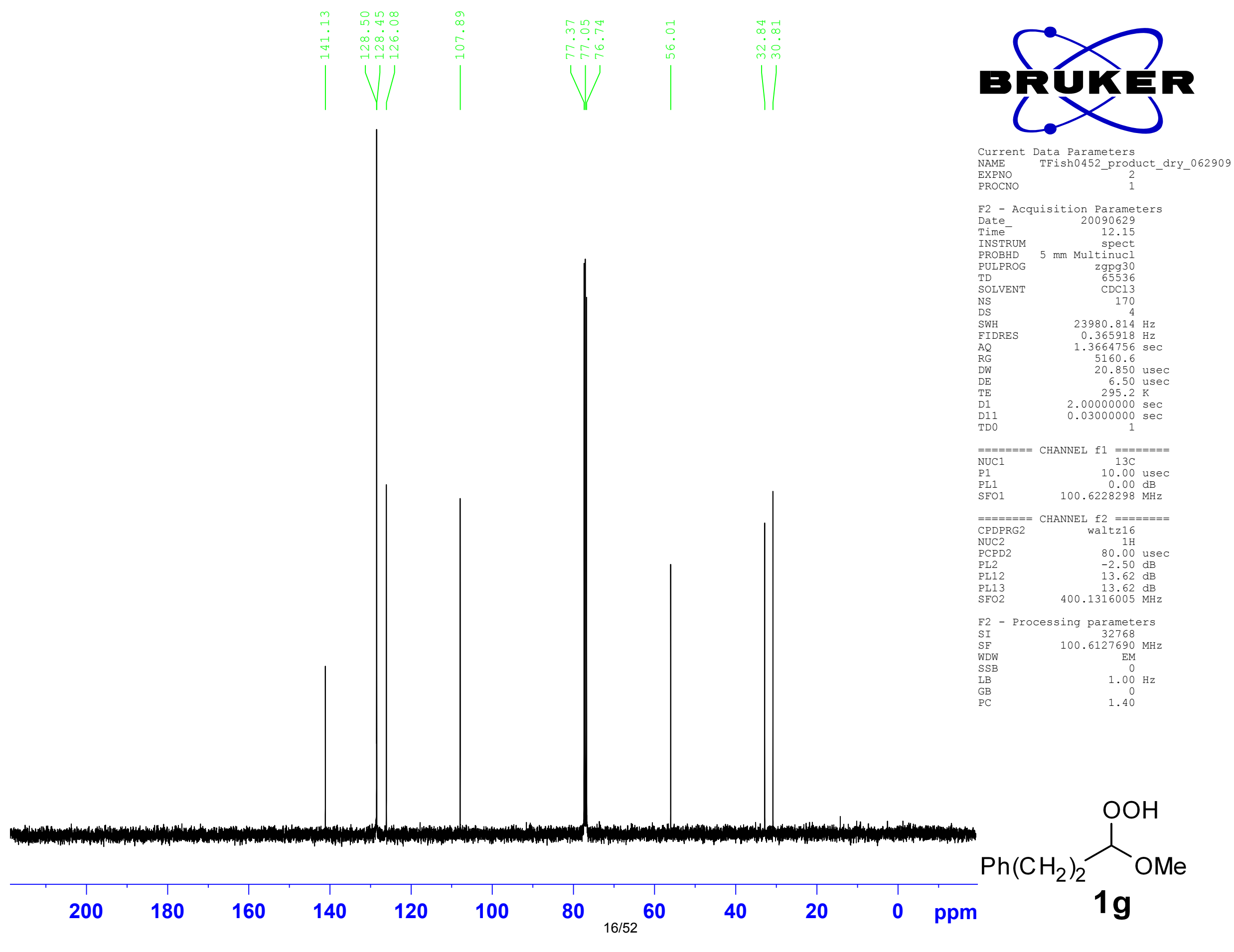




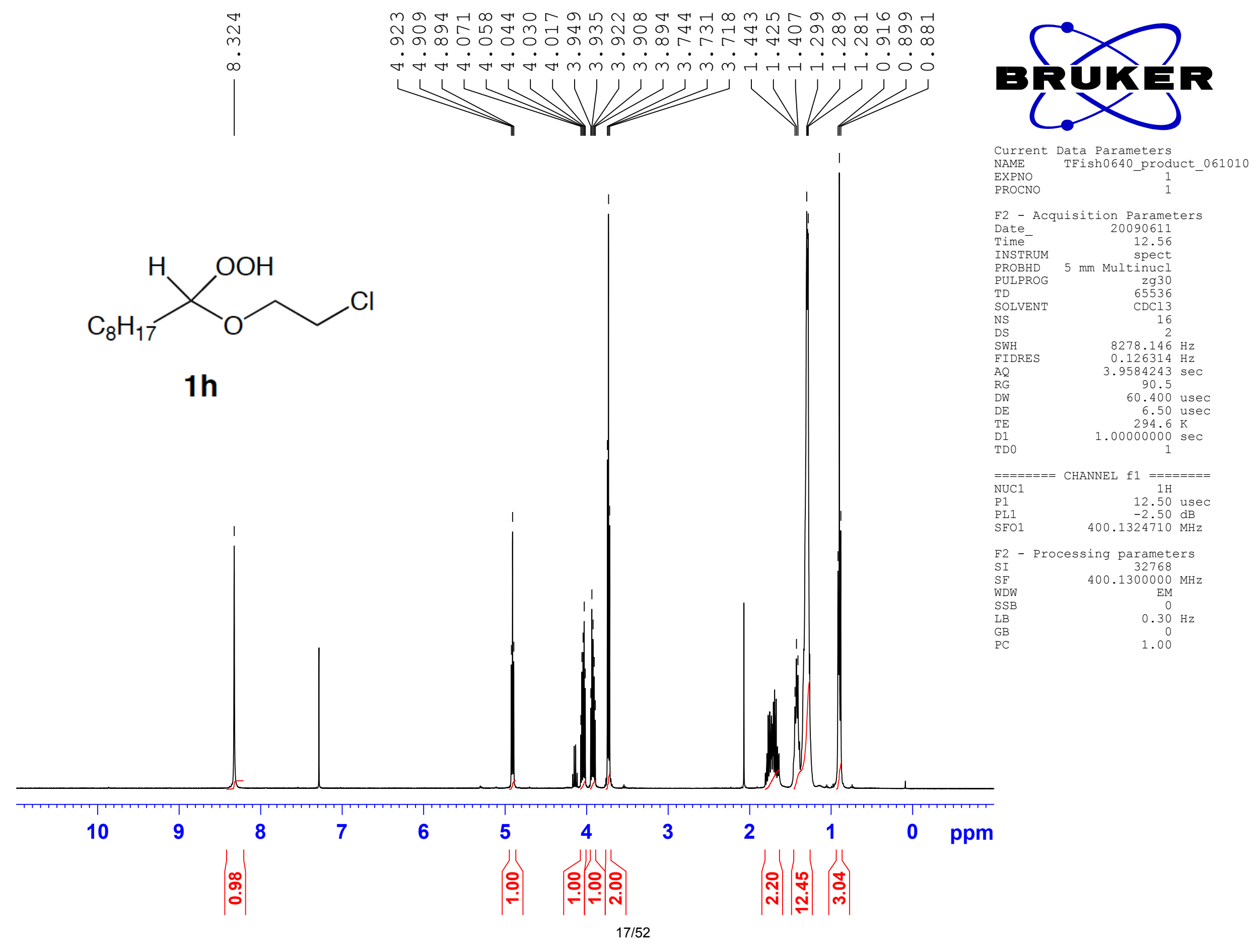




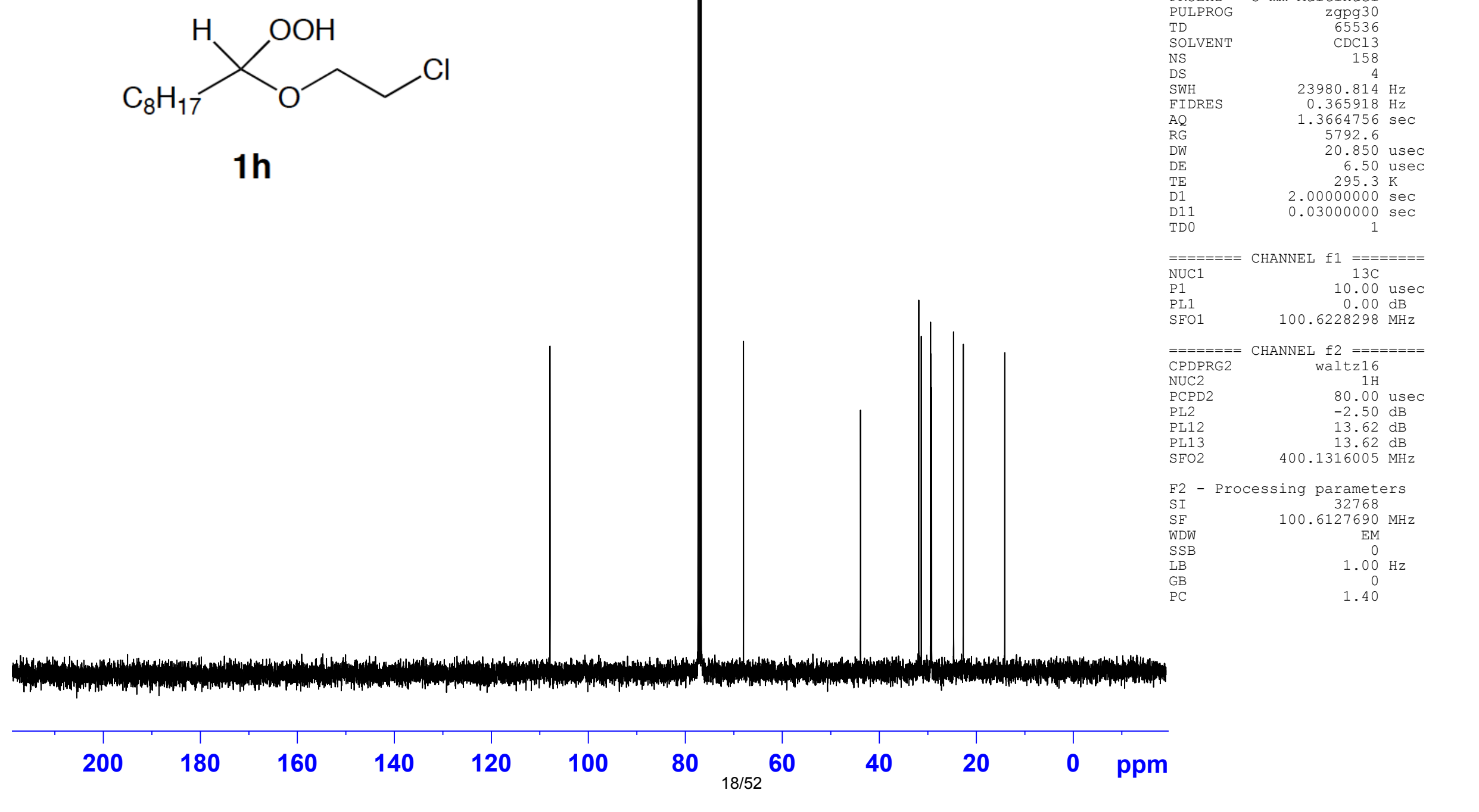



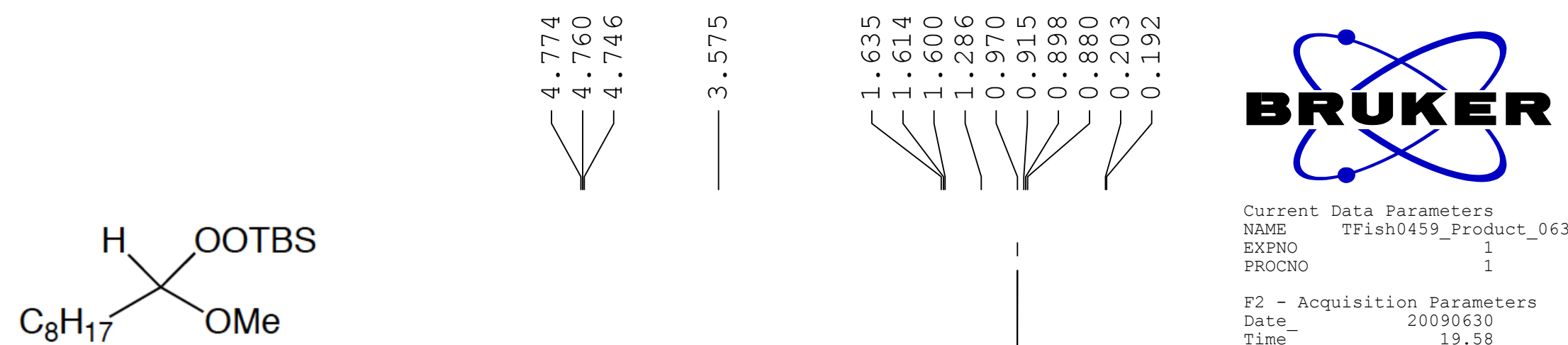

$1 \mathbf{i}$

Current Data Parameters 063009

PROCNO

F2 - Acquisition Parameters

Date__ 20090630

Time 19.58

PROBHD $5 \mathrm{~mm}$ Multinucl

PULPROG $\quad$ zg30

$\begin{array}{lr}\text { PULPROG } & \text { zg30 } \\ \text { TD } & 65536\end{array}$

SOLVENT

DS

SWH

$\begin{array}{ll} & 8278.146 \mathrm{~Hz} \\ \text { FIDRES } & 0.126314 \mathrm{~Hz}\end{array}$

$\mathrm{AQ} \quad 3.9584243 \mathrm{sec}$

RG

$\mathrm{DW}$
$\mathrm{DE}$
$\mathrm{TE}$
$\mathrm{D} 1$

TE
D1
TD0

60.400 usec

6.50 usec

$1.00000000 \mathrm{sec}$

$========$ CHANNEL $\mathrm{fl}========$ NUC1

$\mathrm{P} 1$

PL1

$1 \mathrm{H}$
50 usec

SFO1 $\quad 400.1324710 \mathrm{MHz}$

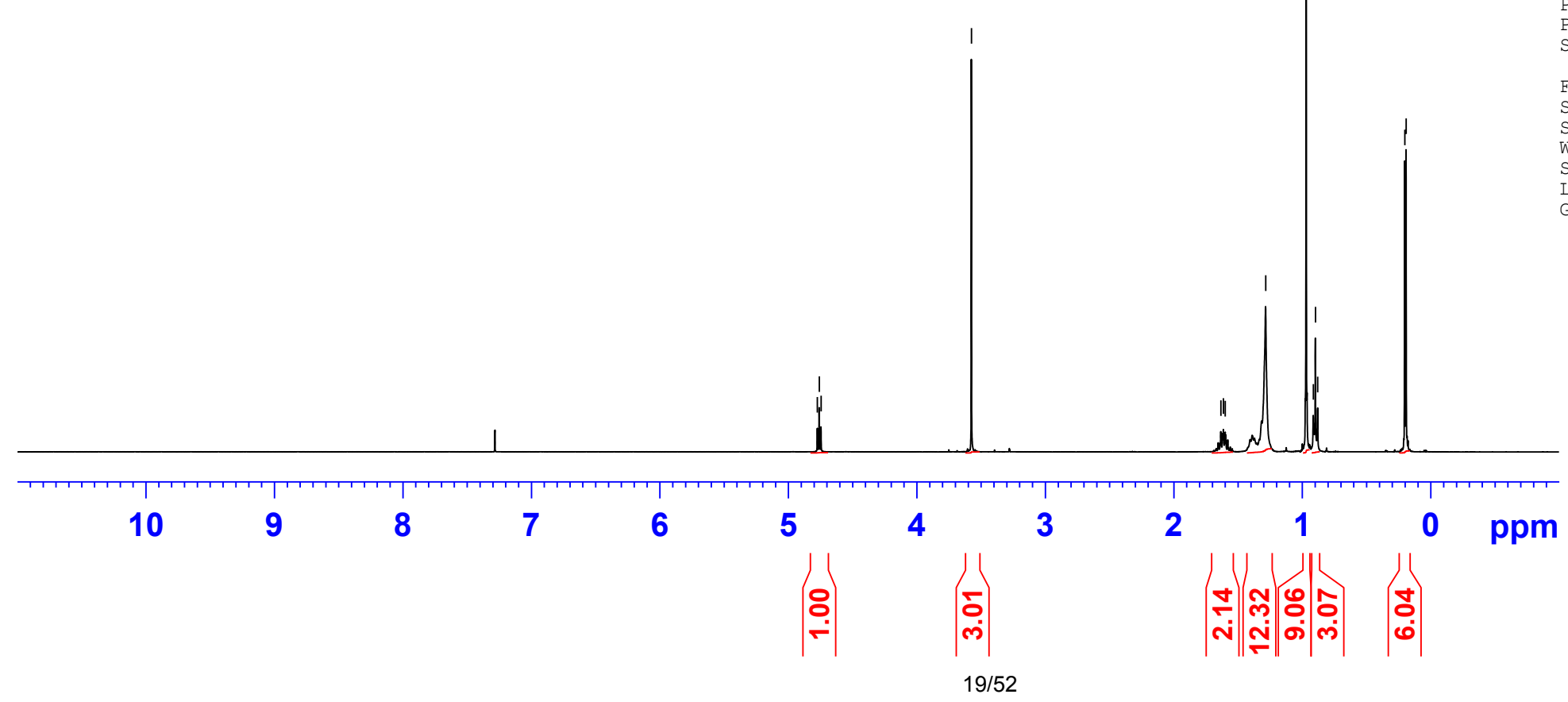

F2 - Processing parameters

SI 32768

SF $\quad 400.1300000 \mathrm{MHz}$

SSB

EM

$0.30 \mathrm{~Hz}$ 


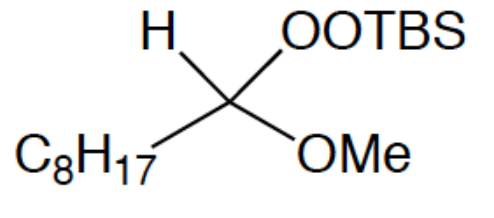

$1 \mathrm{i}$
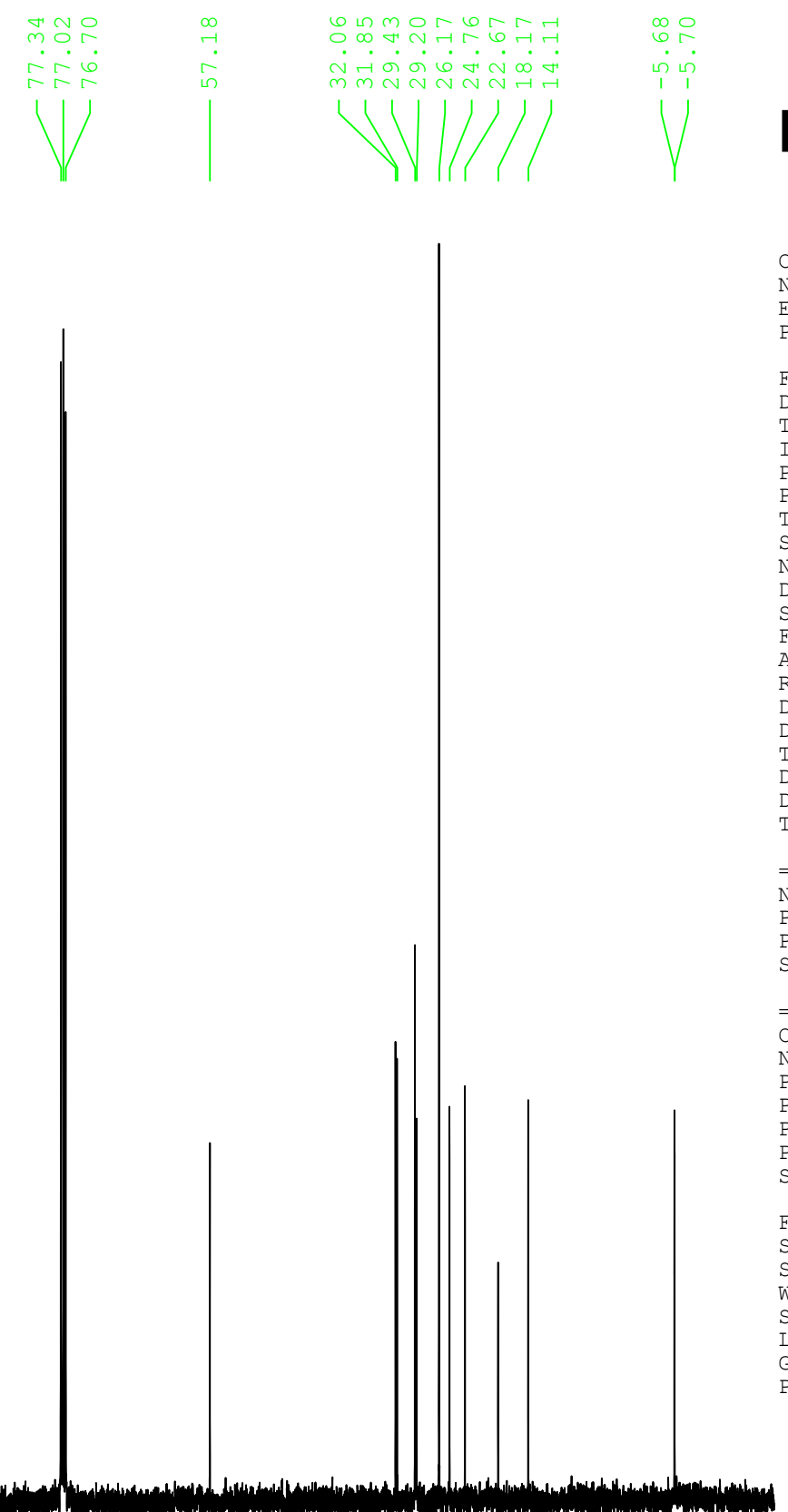

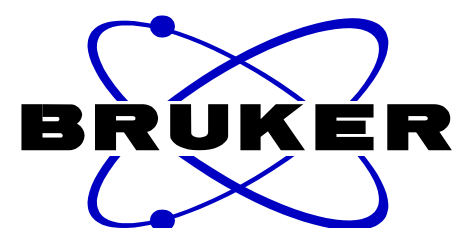

Current Data Parameters TFish0459 Product 063009 EXPNO 2
1

F2 - Acquisition Parameters

Date__ 20090630

Iime 20.02

PROBHD $5 \mathrm{~mm}$ Multinucl

PULPROG zgpg30

OLVENT

SWH

Q

RG

DE

D1

TDO

NUC1

P1 1

$\mathrm{PL} 1$
$\mathrm{SFO1}$

65536

165
4
$23980.814 \mathrm{~Hz}$

$0.365918 \mathrm{~Hz}$

$1.3664756 \mathrm{sec}$

4597.6

20.850 usec

6.50 us

$.00000000 \mathrm{sec}$

$0.03000000 \mathrm{sec}$

$=$ CHANNEL $\mathrm{f} 1$

$=======$ CHANNEL f $2========$

(1)

NUC2 2
PCPD2

PL2

SFO2

$400.1316005 \mathrm{MHz}$

2 - Processing parameters

32768

$\quad 100.6127690 \mathrm{MHz}$

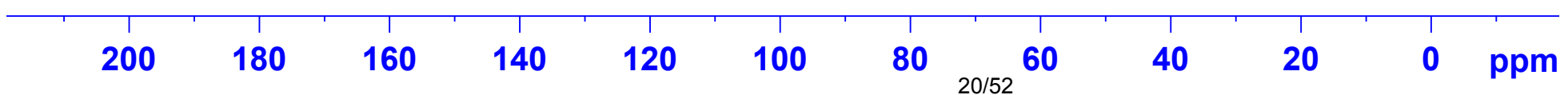


G 드

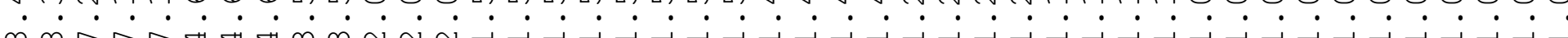

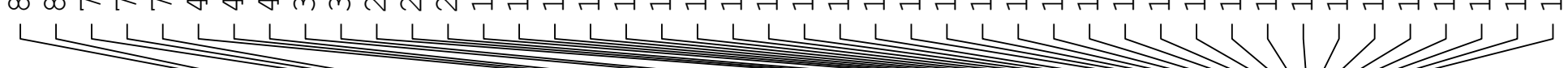
17
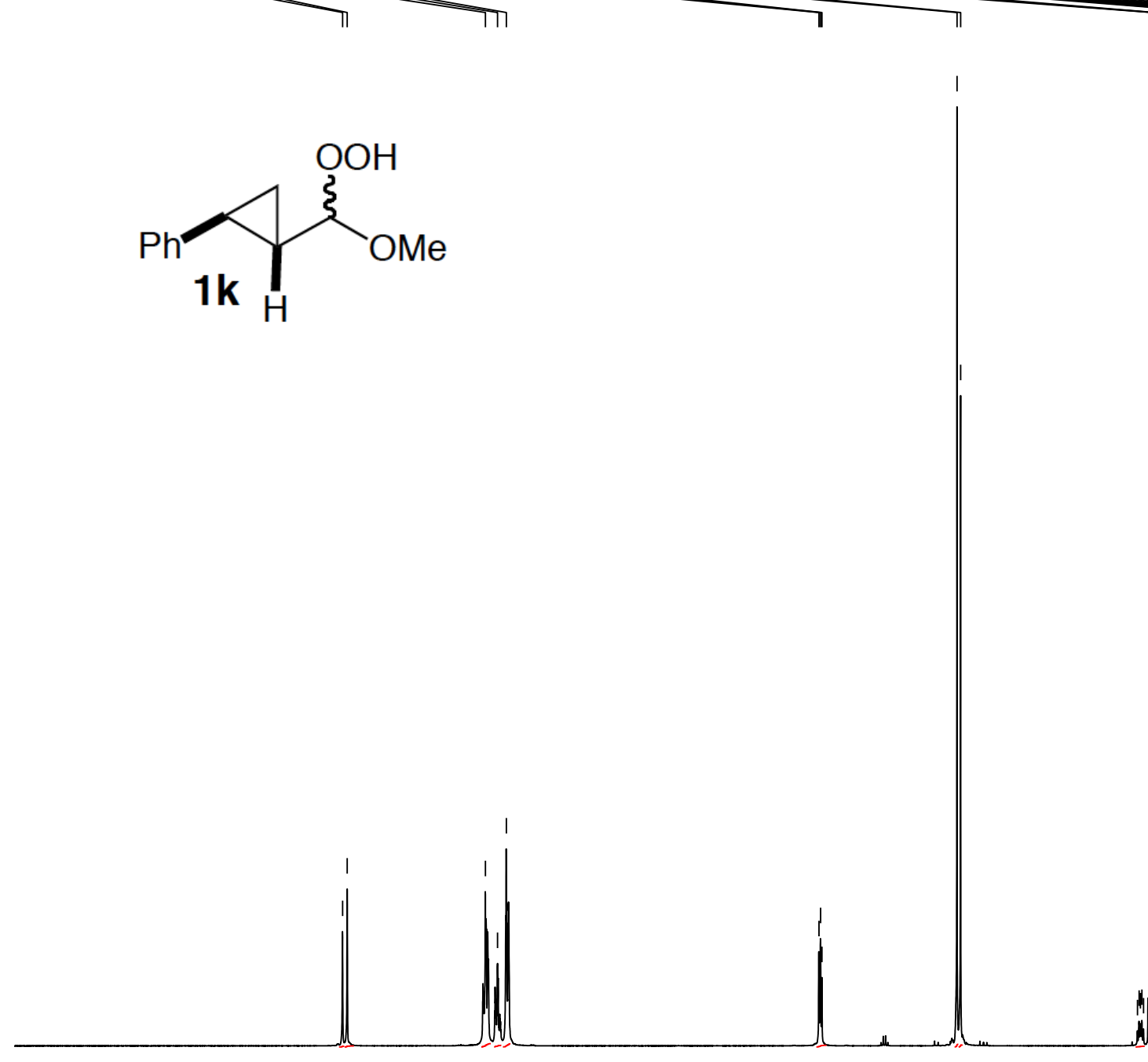

\begin{abstract}
9
\end{abstract}

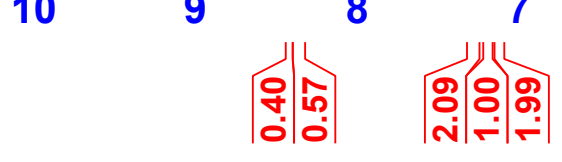
6

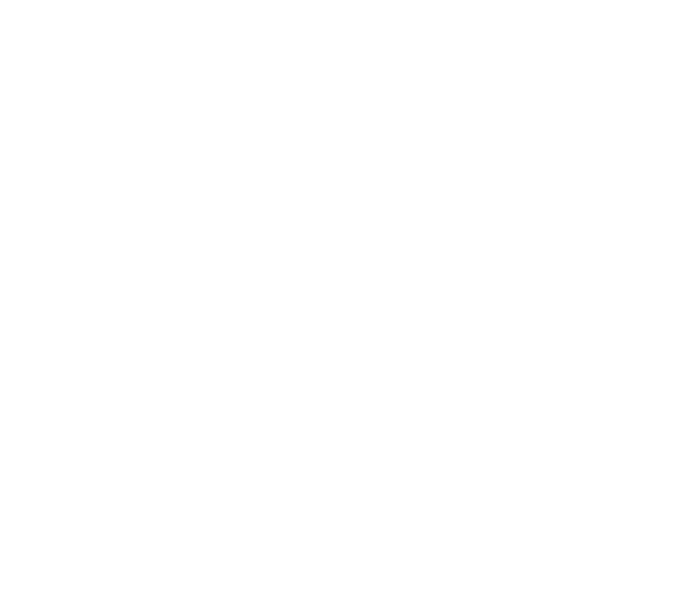

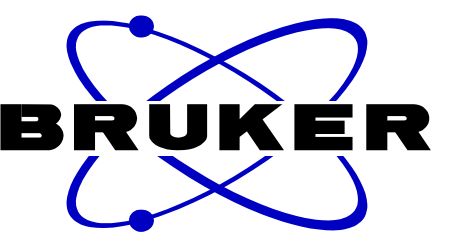

Current Data Parameters TFish0529 product 111409 PROCNO

F2 - Acquisition Parameters Date_ 20091114 Time 17.54

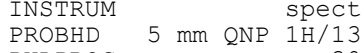
PULPROG
zg 30 $\begin{array}{lr}\text { TD } & \text { zg30 } \\ \text { TD } & 65536\end{array}$ DS SWH $\quad 8278.146 \mathrm{~Hz}$ FIDRES $\quad 0.126314 \mathrm{~Hz}$ AQ $\quad 3.9584243 \mathrm{sec}$ RG $\mathrm{DW}$
$\mathrm{DE}$
$\mathrm{TE}$ TE 90.5
60.400 usec 6.50 usec $1.00000000 \mathrm{sec}$ $\begin{array}{lr}=======\text { CHANNEL } \mathrm{f} 1======= \\ \text { NUC1 } 1 \mathrm{H} \\ \text { P1 } & 12.00 \text { usec } \\ \text { PL1 } & -3.35 \mathrm{~dB} \\ \text { SFO1 } & 400.1324710 \mathrm{MHz}\end{array}$ F2 - Processing parameters SI 32768 SF $\quad 400.1300000$ WDW SSB
LB $\mathrm{GB}$
$\mathrm{PC}$ 400.1300000
$\mathrm{EM}$ $\mathrm{EM}$
0
0
0
30 $0.30 \mathrm{~Hz}$ 1.00 |

3

1

1

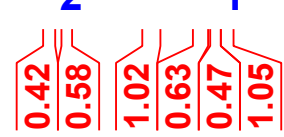




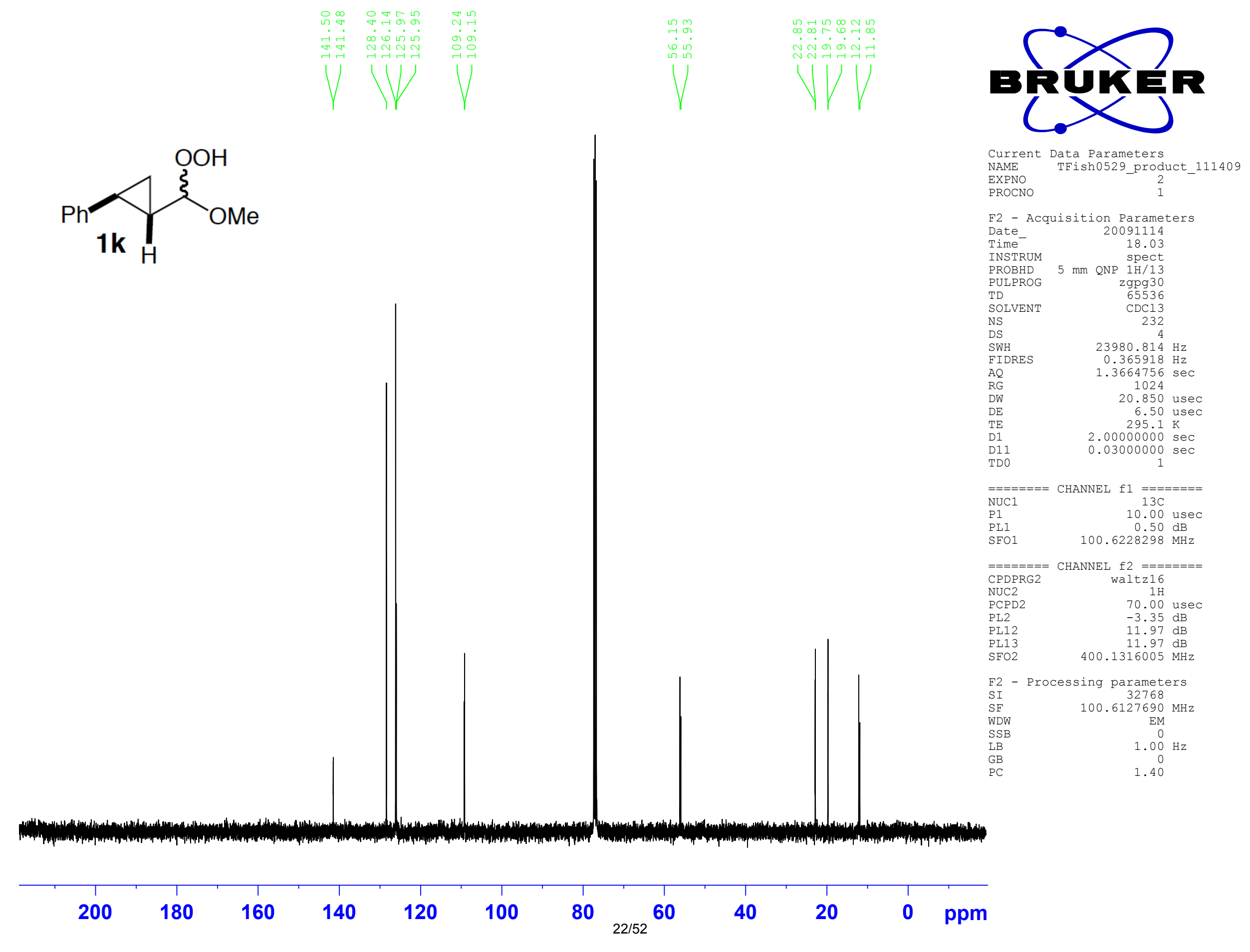




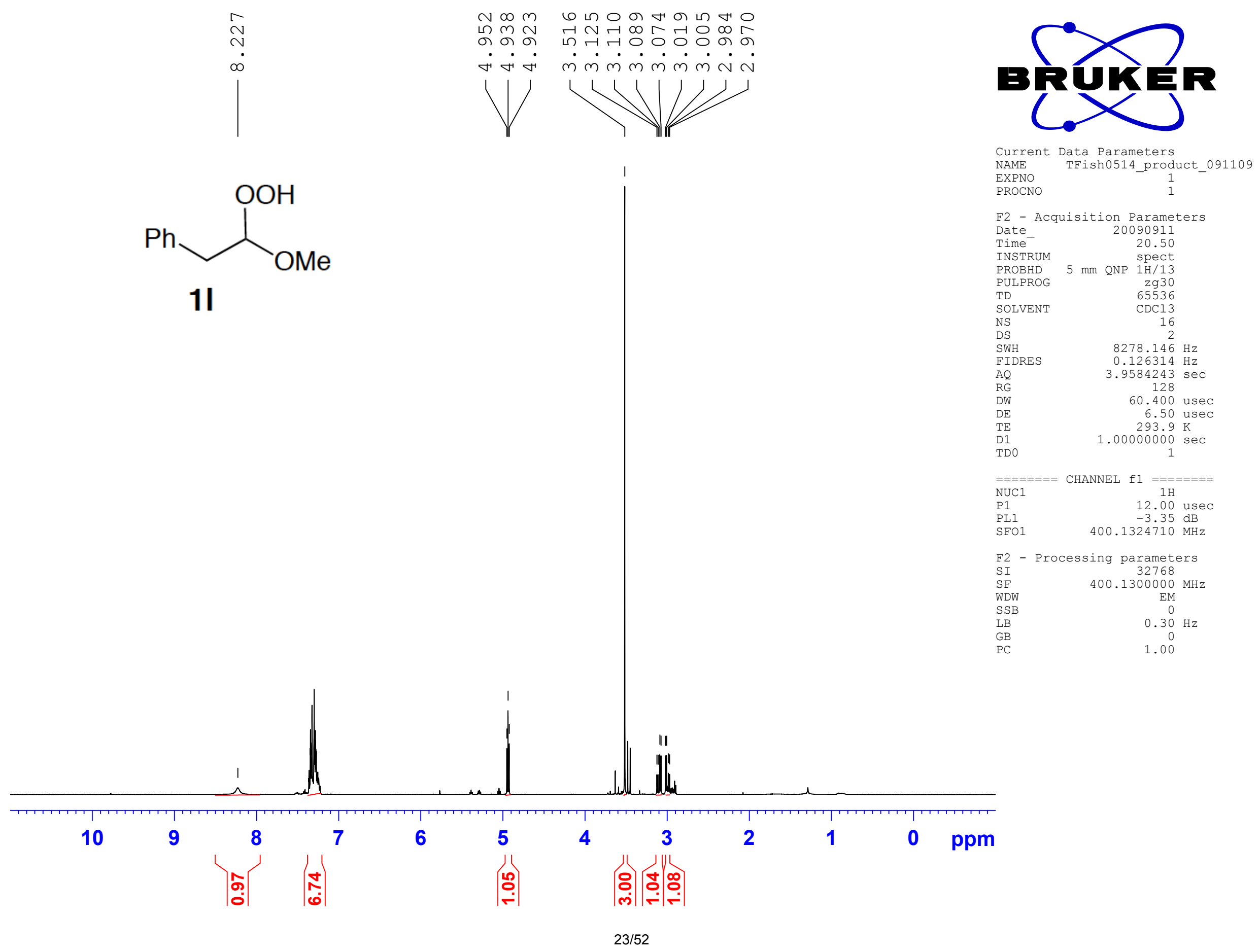




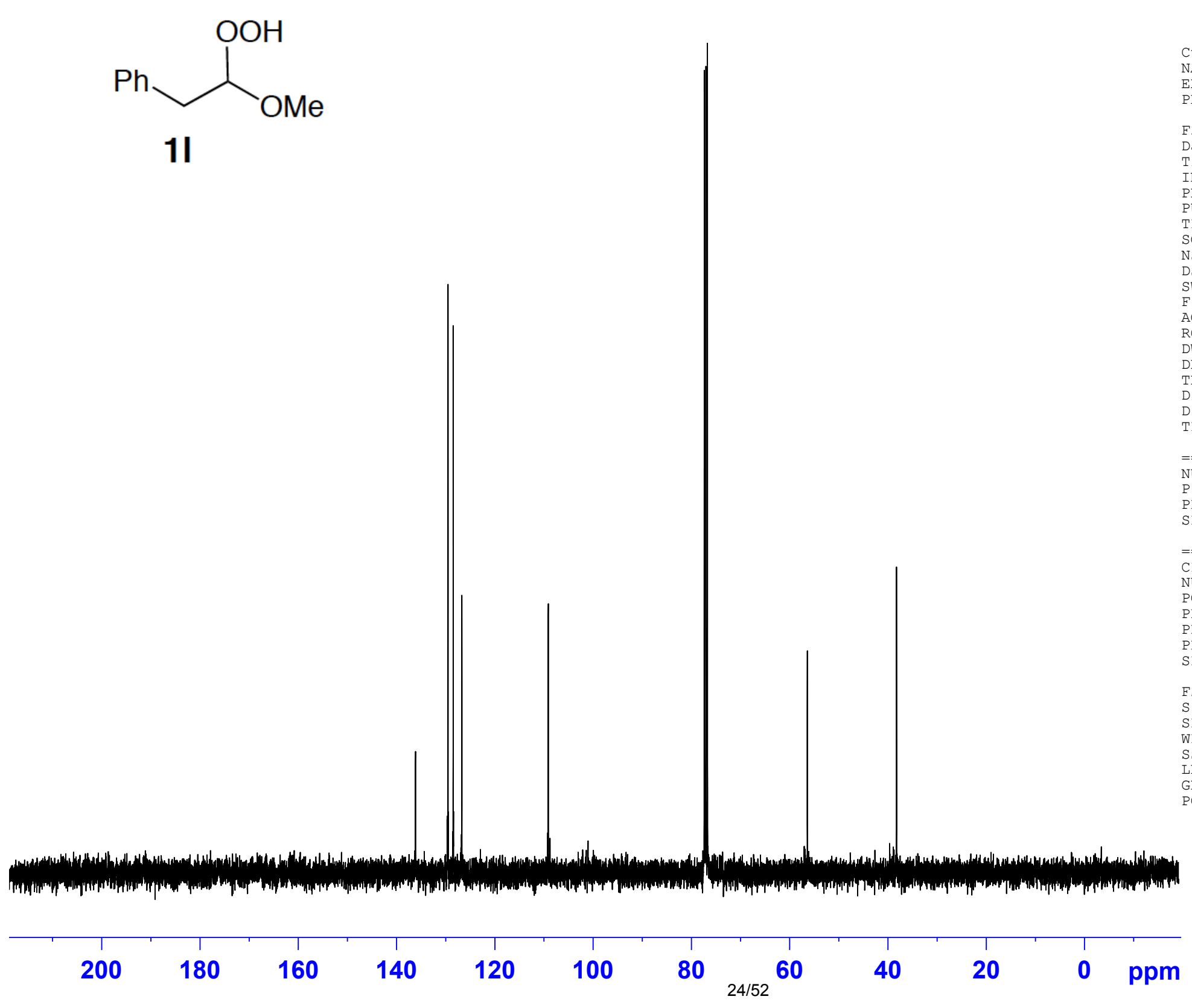

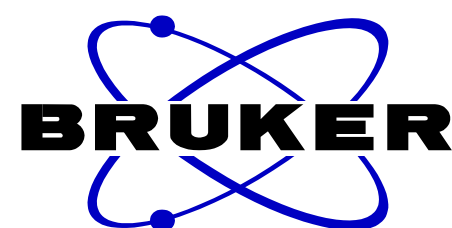

Current Data Parameters NAME TFish0514_product_091109 EXPNO

F2 - Acquisition Parameters Date__ 2009091 $\begin{array}{ll}\text { Time } & 20.57 \\ \text { INSTRUM } & \text { spect }\end{array}$ PROBHD $5 \mathrm{~mm}$ QNP 1H/13 zgpg30

SOLVENT

DS $23980.814 \mathrm{~Hz}$
SWH 23980.814
FIDRES $\quad 0.365918 \mathrm{~Hz}$ $1.3664756 \mathrm{sec}$ 1024

20.850 usec
6.50 usec $2.00000000 \mathrm{~K}$ $2.0000000 \mathrm{sec}$ TDO 1 sec $=======$ CHANNEL $\mathrm{f} 1$

NUC1 P1 1

SFO1 $13 \mathrm{C}$ .00 usec

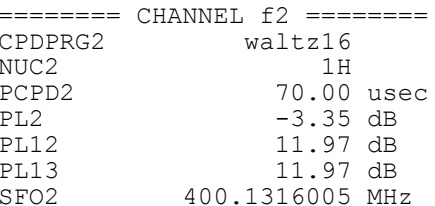

2 - Processing parameters

32768

$\begin{array}{cc}\text { SE } & 100.6127690 \mathrm{MHz}\end{array}$

$S S B$

B

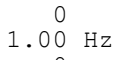

1.0 


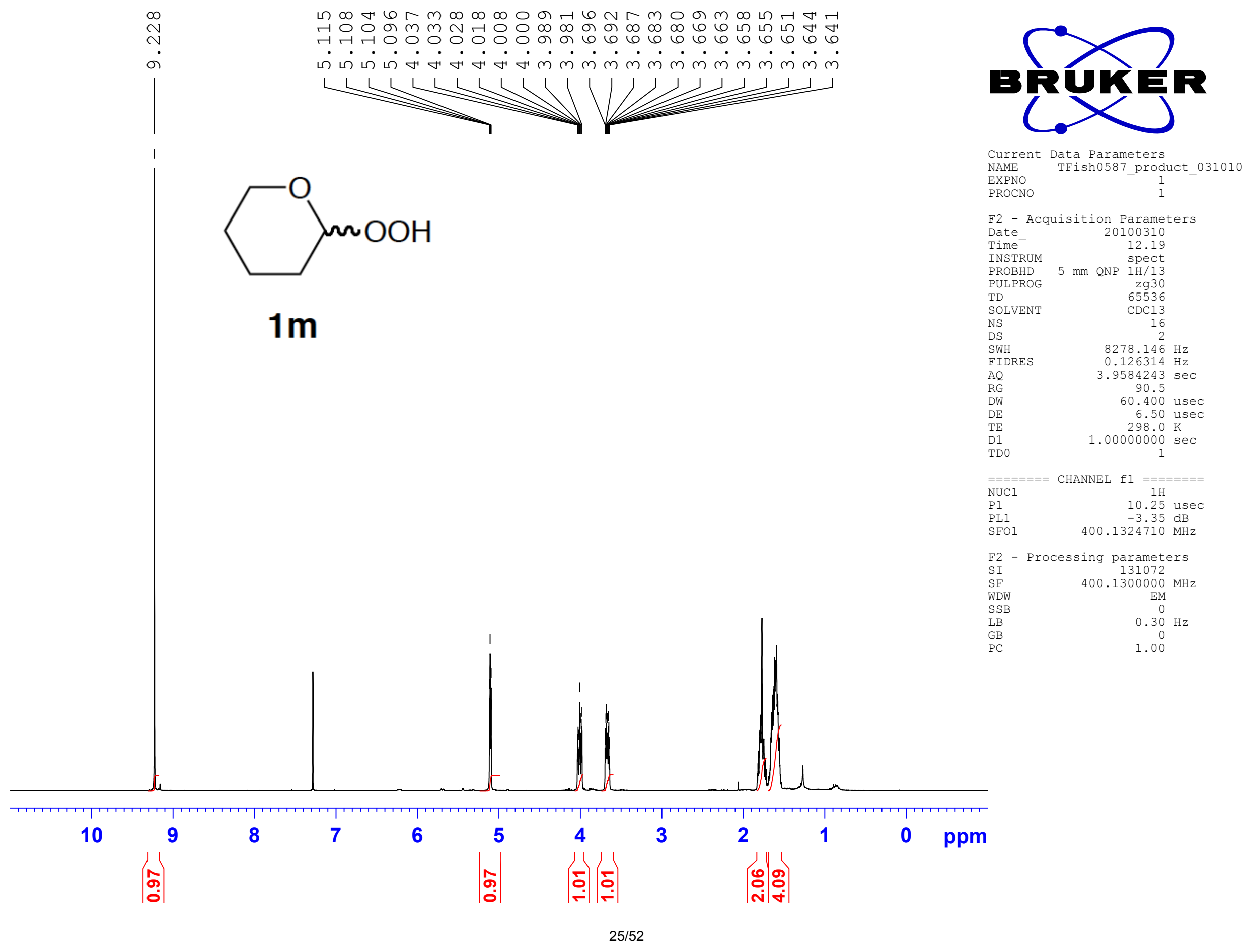




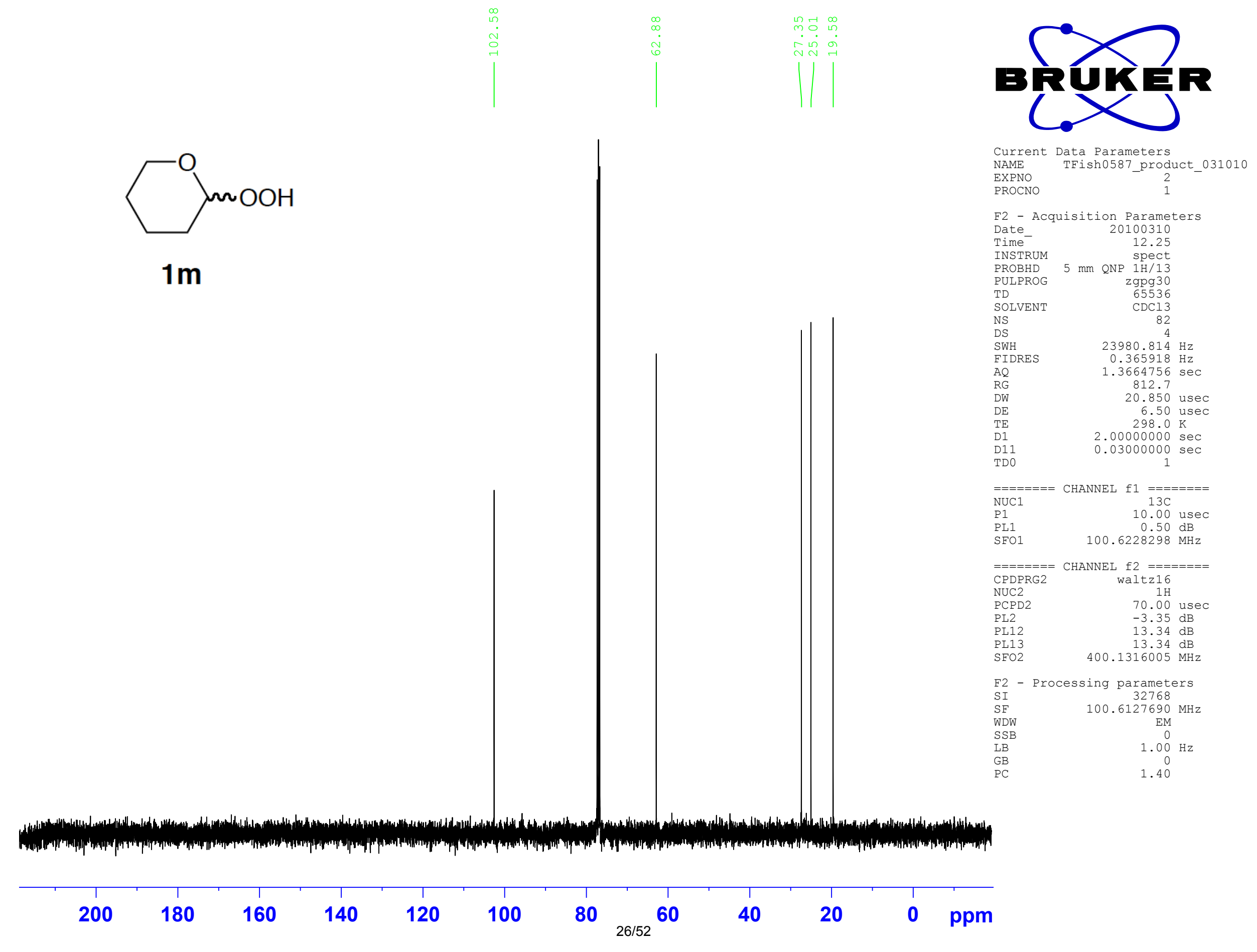




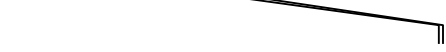

1n
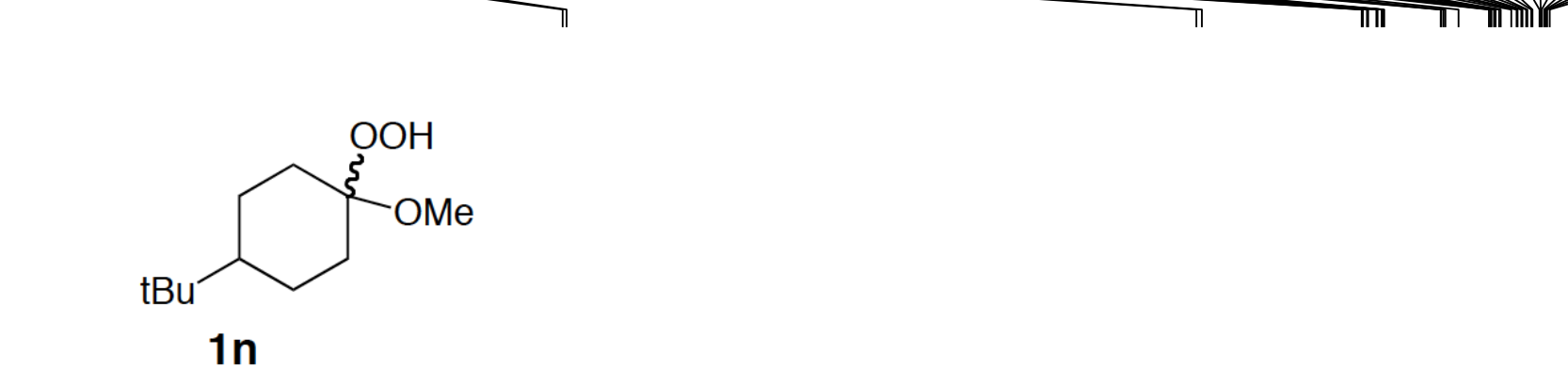

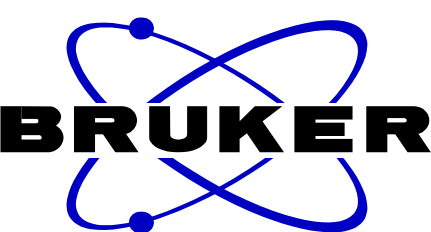

Current Data Parameters NAME TFish0580 product_030310 EXPNO PROCNO

F2 - Acquisition Parameters Date__ 20100303 Time 19.46 $\begin{array}{ll}\text { INSTRUM } & \text { spect } \\ \text { PROBHD } & 5 \mathrm{~mm} \text { QNP } 1 \mathrm{H} / 13 \\ \text { PUITPROG } & \end{array}$ $\begin{array}{lr}\text { PULPROG } & \mathrm{zg} 30 \\ \text { TD } & 65536\end{array}$ TDLVENT DS SWH $\quad 8278.146 \mathrm{~Hz}$ $\begin{array}{lr}\text { FIDRES } & 0.126314 \mathrm{~Hz} \\ \text { AQ } & 3.9584243 \mathrm{sec}\end{array}$ RG DW $\mathrm{TE}$
$\mathrm{D} 1$ TD0 90.5
60.400 usec 6.50 usec $1.00000000 \mathrm{sec}$ $=======$ CHANNEL $\mathrm{f} 1 \mathrm{l}=======$ NUC1 $\mathrm{P} 1$ $\begin{array}{lr}\text { PL1 } & -3.35 \mathrm{~dB} \\ \text { SFO1 } & 400.1324710 \mathrm{MHz}\end{array}$ 00 usec

F2 - Processing parameters SI 65536 SF $\quad 400.1300000 \mathrm{MHz}$ EM 0
$0.30 \mathrm{~Hz}$ 1.00

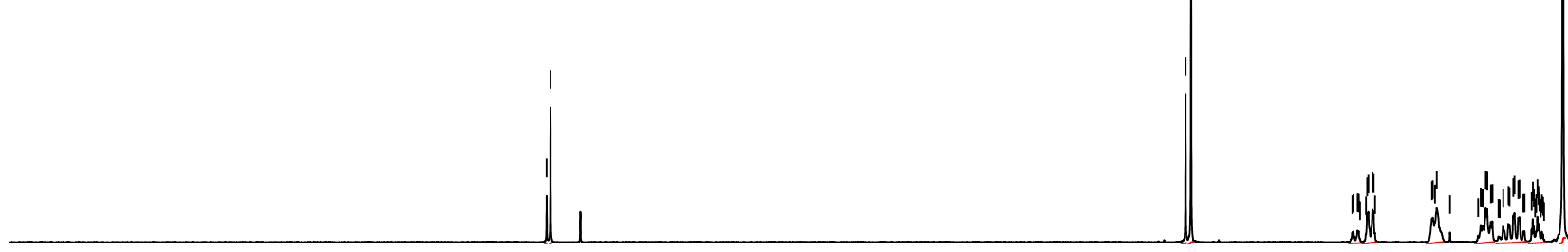

6

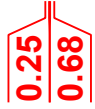

5

4

3

2
1

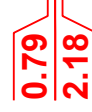
華 

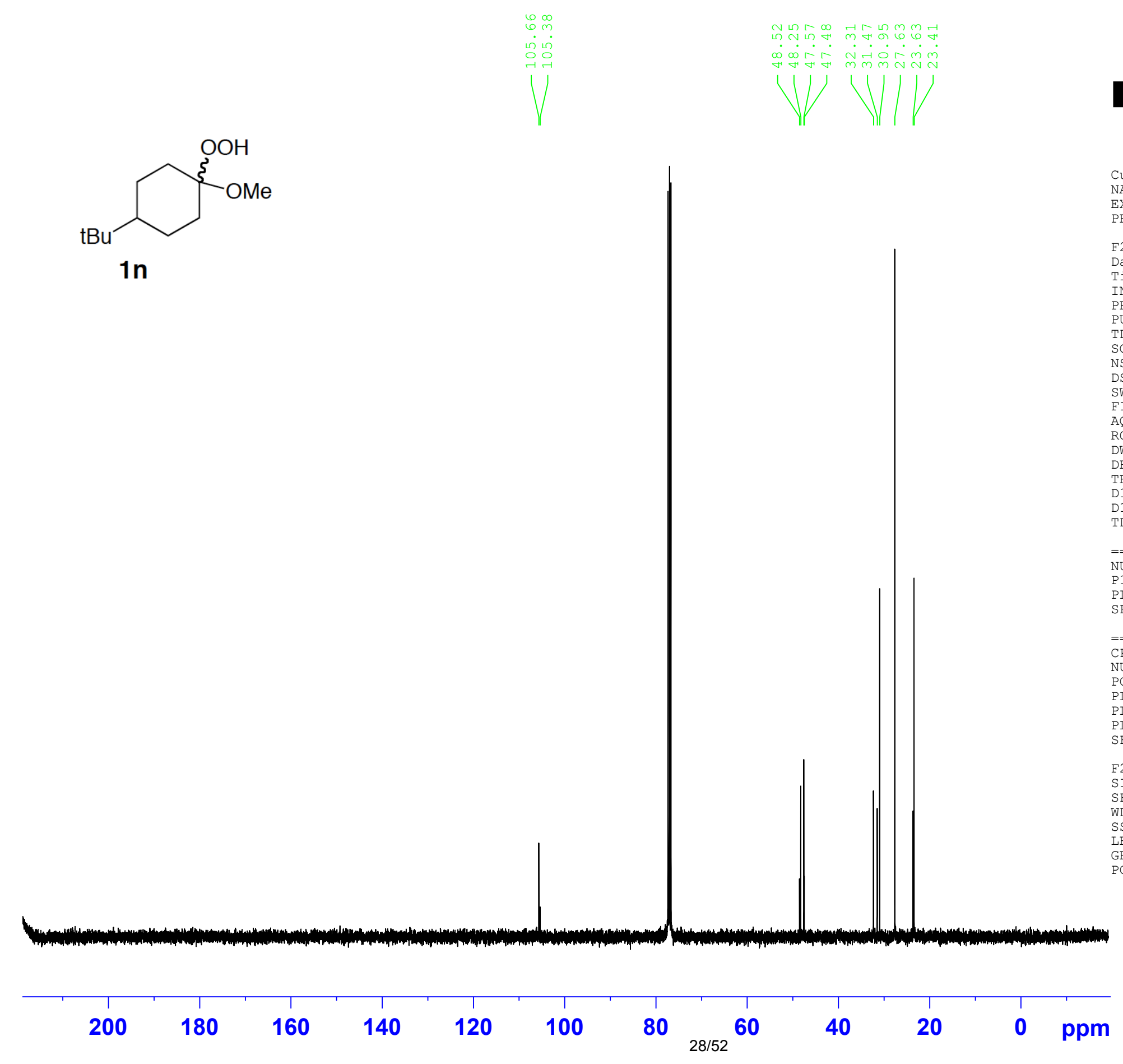

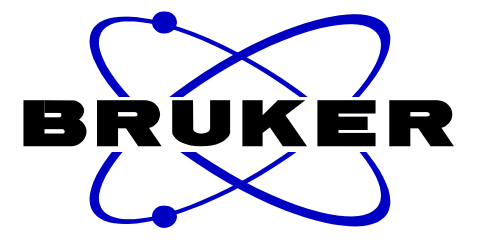

Current Data Parameters 030310 EXPNO

Fish0580_product_030310 PROCNO

F2 - Acquisition Parameters

Date__ 20100303

$\begin{array}{ll}\text { Time } & 19.56 \\ \text { INSTRUM } & \text { spect }\end{array}$

PROBHD $5 \mathrm{~mm}$ QNP $1 \mathrm{H} / 13$

PULPROG zgpg30

SOLVENT

S

SWH

MQ
MDES

RG

DE

D1
D11
TD0

DO

$=====$
NUC1

P1

PL1

65536
$\mathrm{CDC} 13$

4
$23980.814 \mathrm{~Hz}$

$0.365918 \mathrm{~Hz}$

$1.3664756 \mathrm{sec}$

1149.4

20.850 usec

6.50 us

$2.00000000 \mathrm{sec}$

$0.03000000 \mathrm{sec}$

$=$ CHANNEL $\mathrm{f} 1$

$======$

NUC2
PCPD2
PL2

L 2

$\mathrm{PL} 13$
$13 \mathrm{C}$
10.00 usec

$0.50 \mathrm{~dB}$
$100.6228298 \mathrm{MHz}$

CHANNEL $\mathrm{f} 2$

waltz16

70.00 usec

$-3.35 \mathrm{~dB}$

$11.97 \mathrm{~dB}$

$400.1316005 \mathrm{MHz}$

2 - Processing parameters

65536

$100.6127690 \mathrm{MHz}$

WDW

GB

PC 


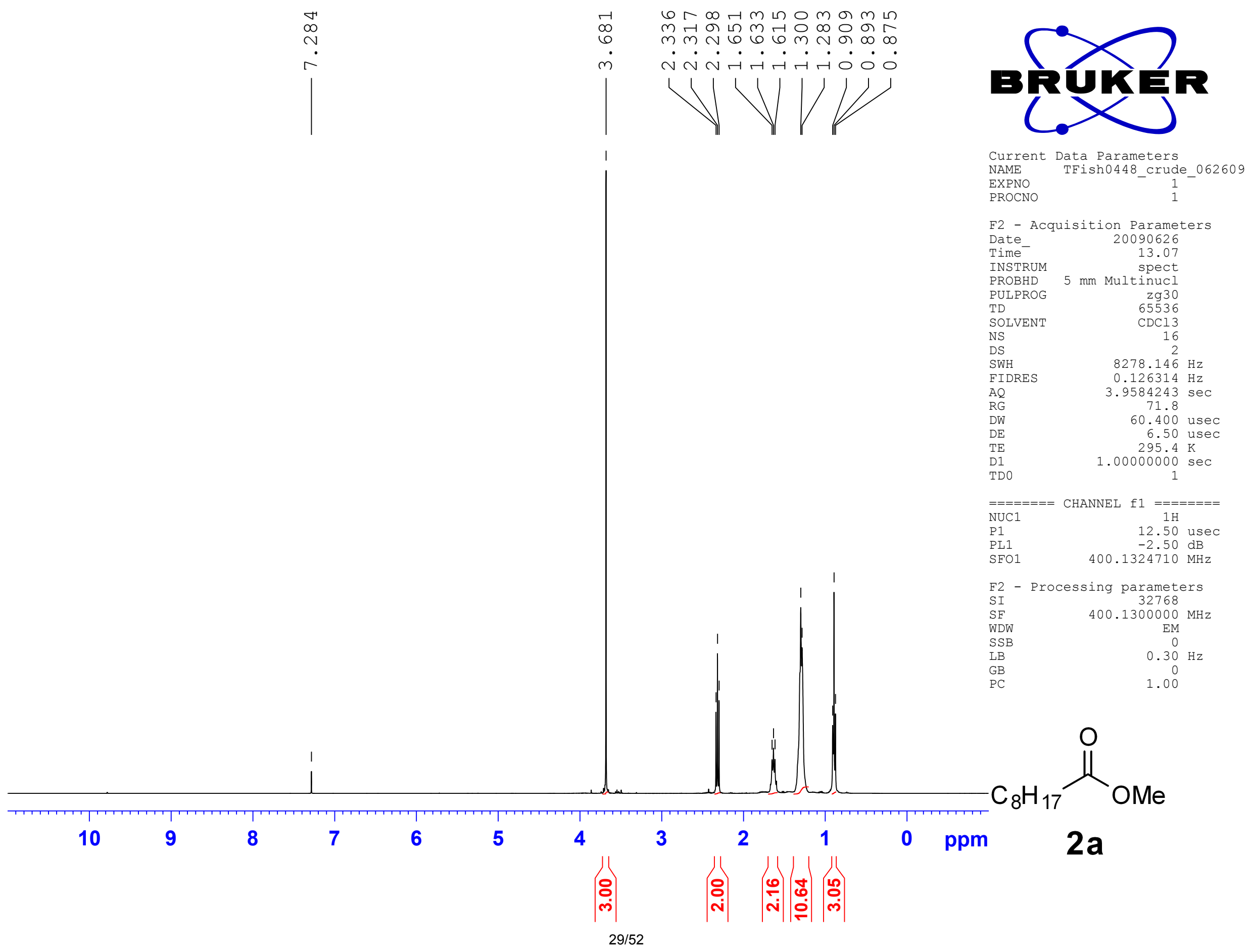




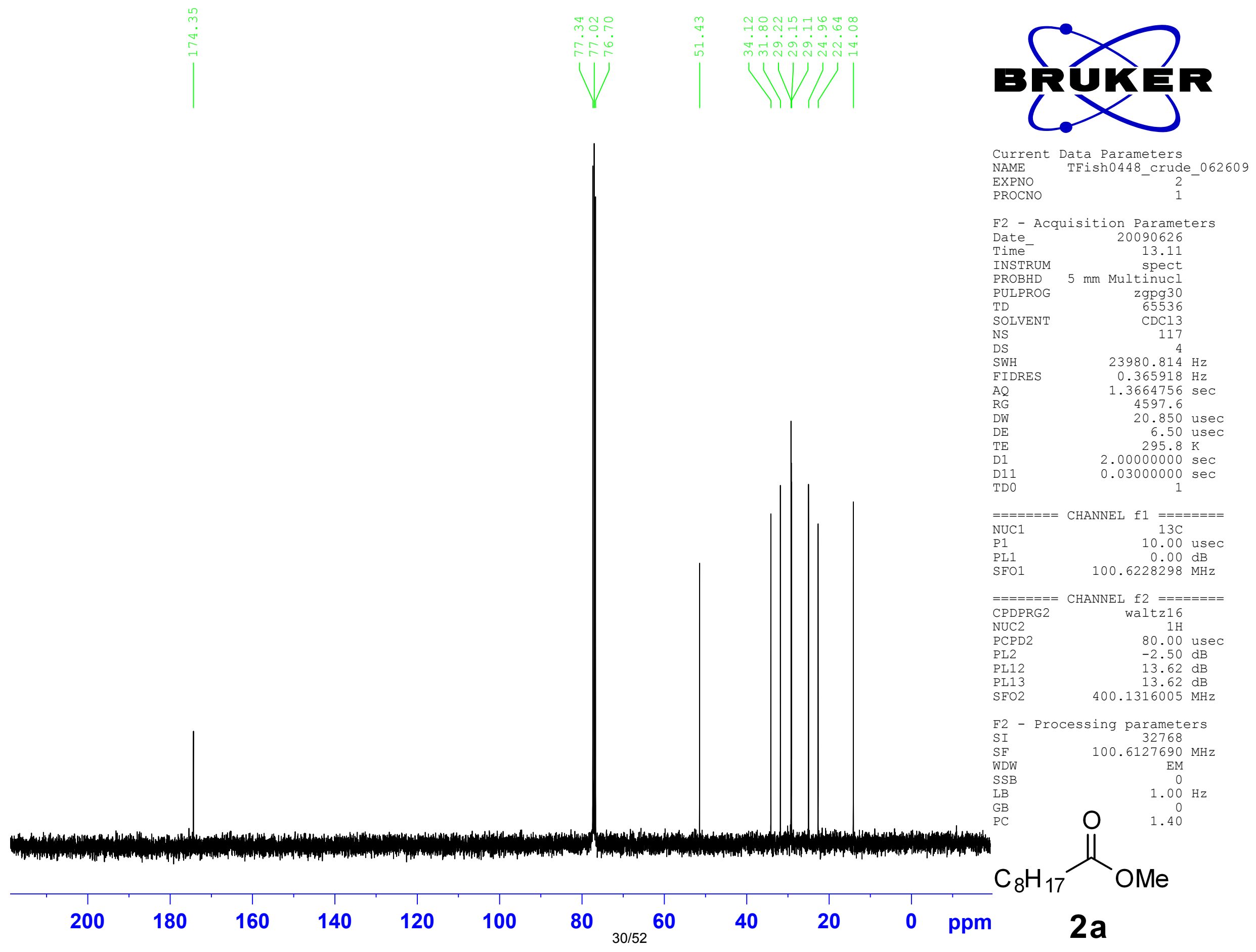




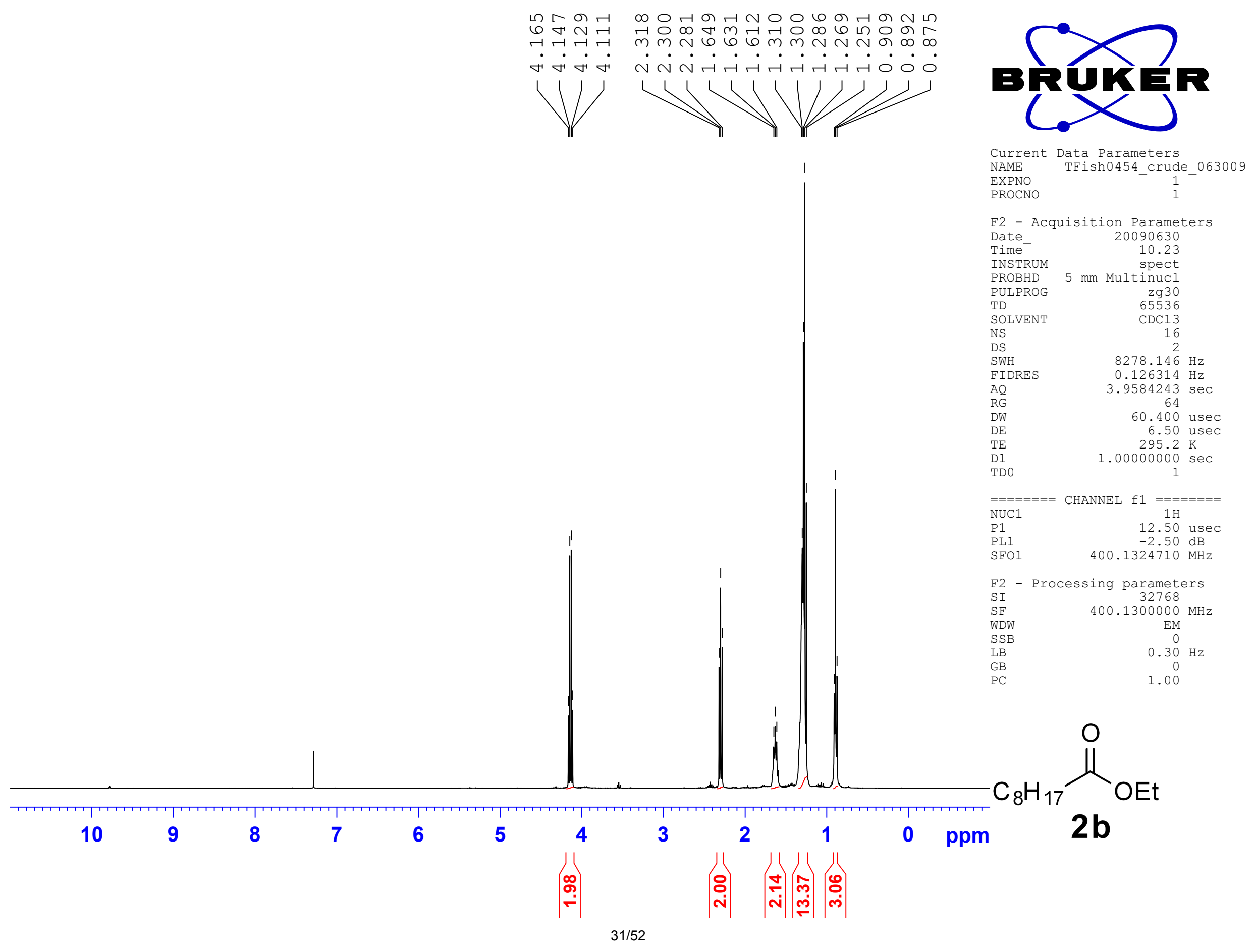




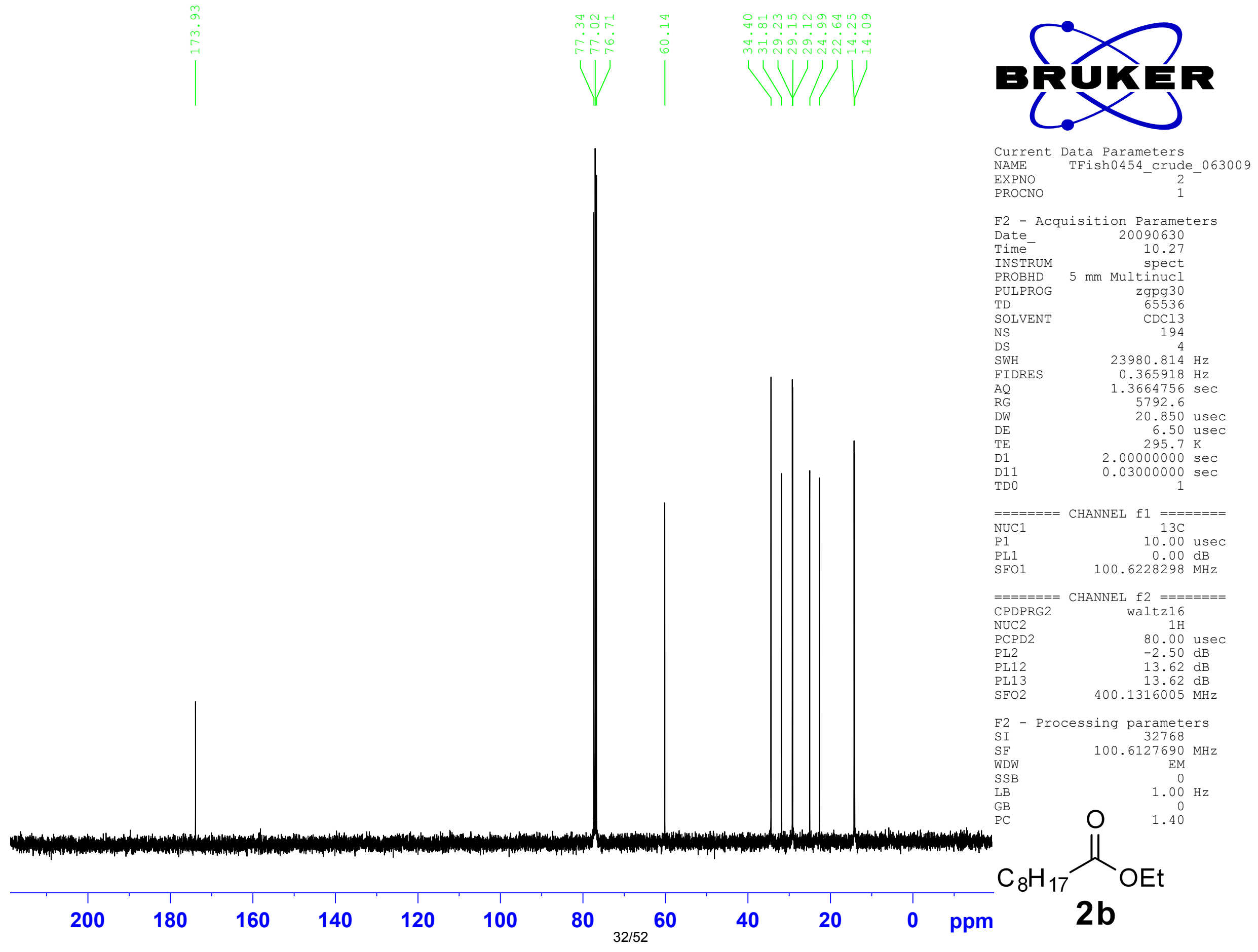




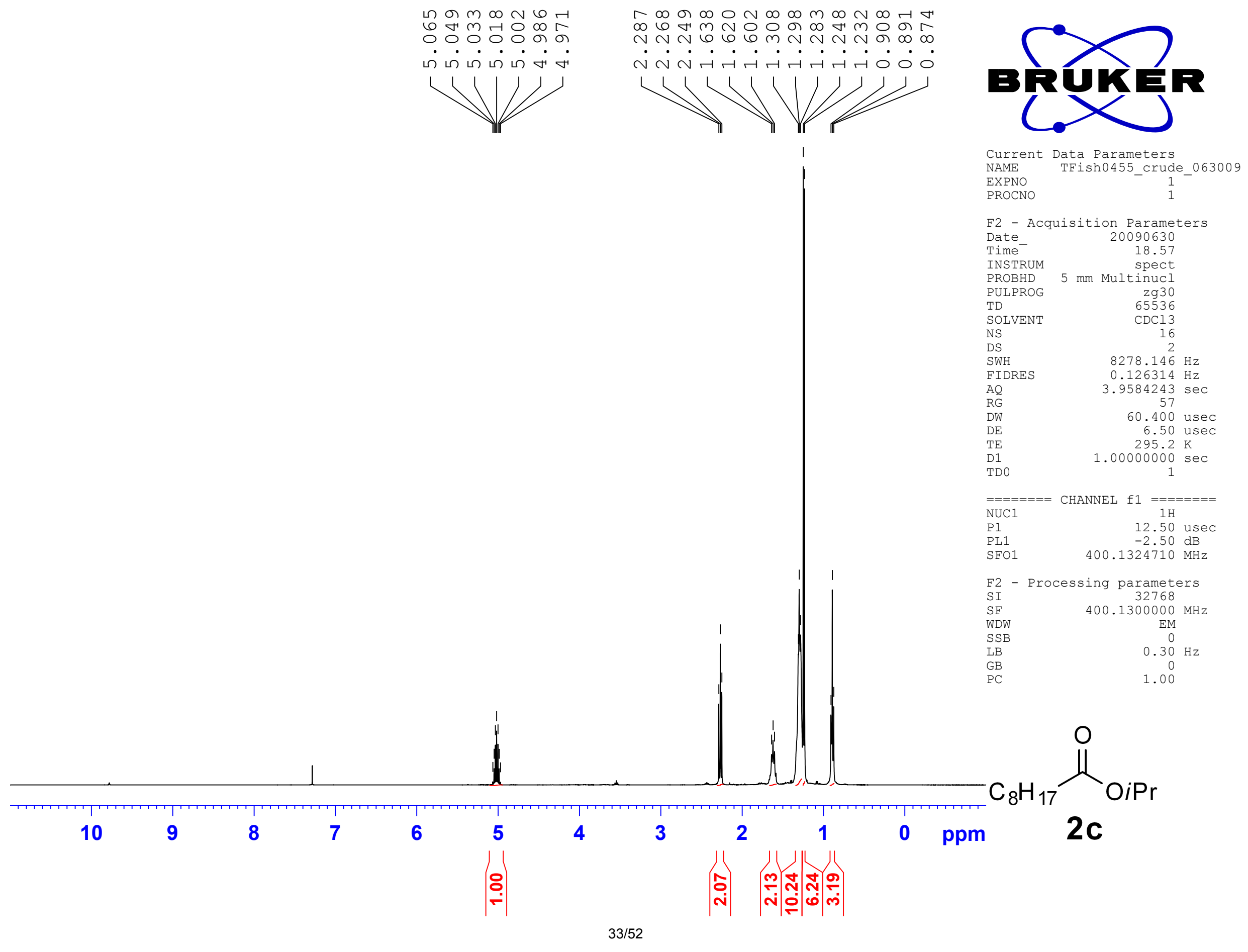




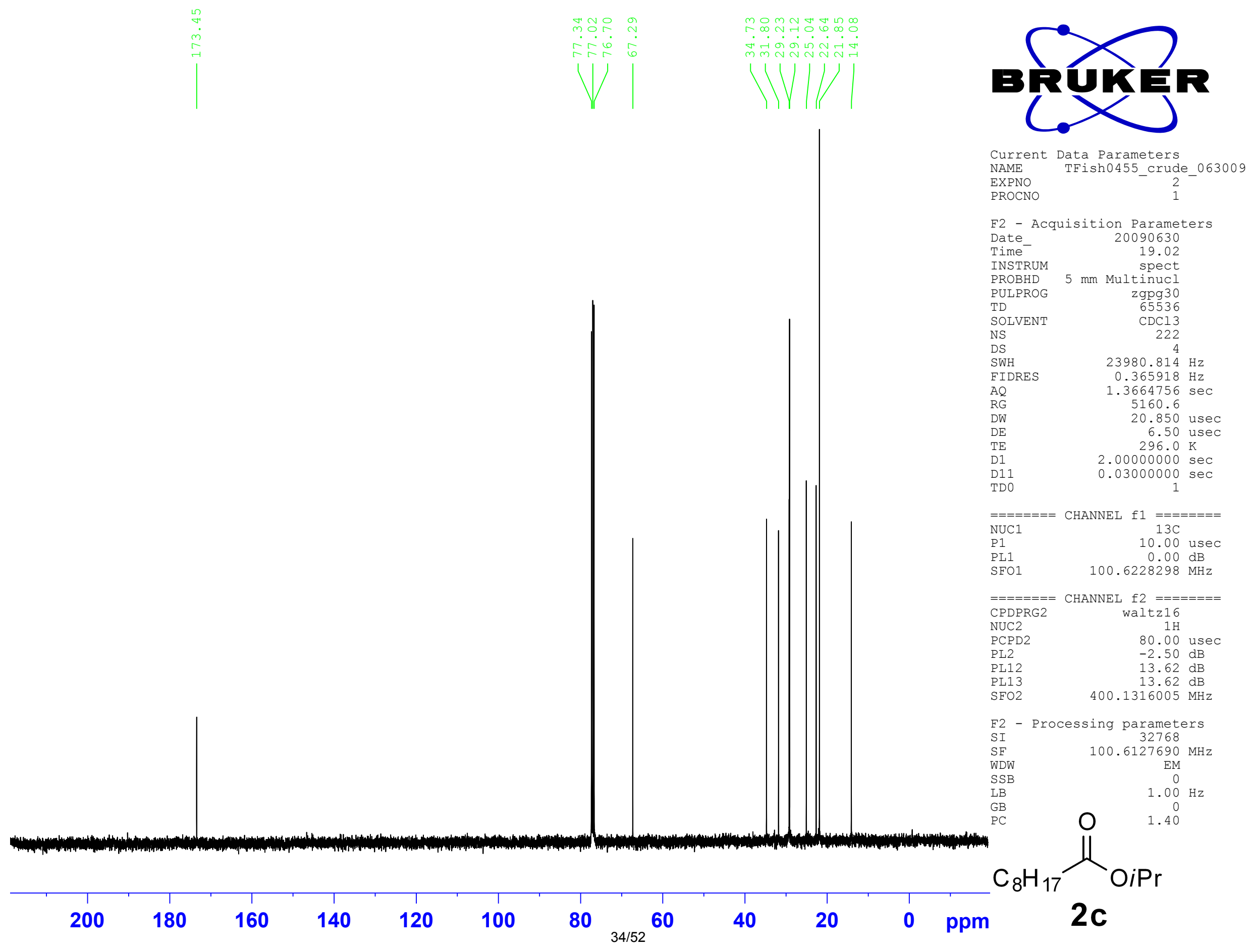


- の

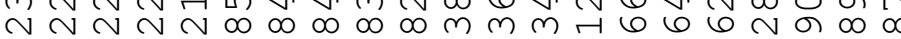
$\dot{\sigma} \dot{\sigma} \dot{\sigma} \dot{\sim} \dot{m} \dot{m} \dot{m} \dot{N} \dot{N} \dot{N} \dot{\sim} \dot{r} \dot{r} \dot{H} \dot{0} \dot{0}$
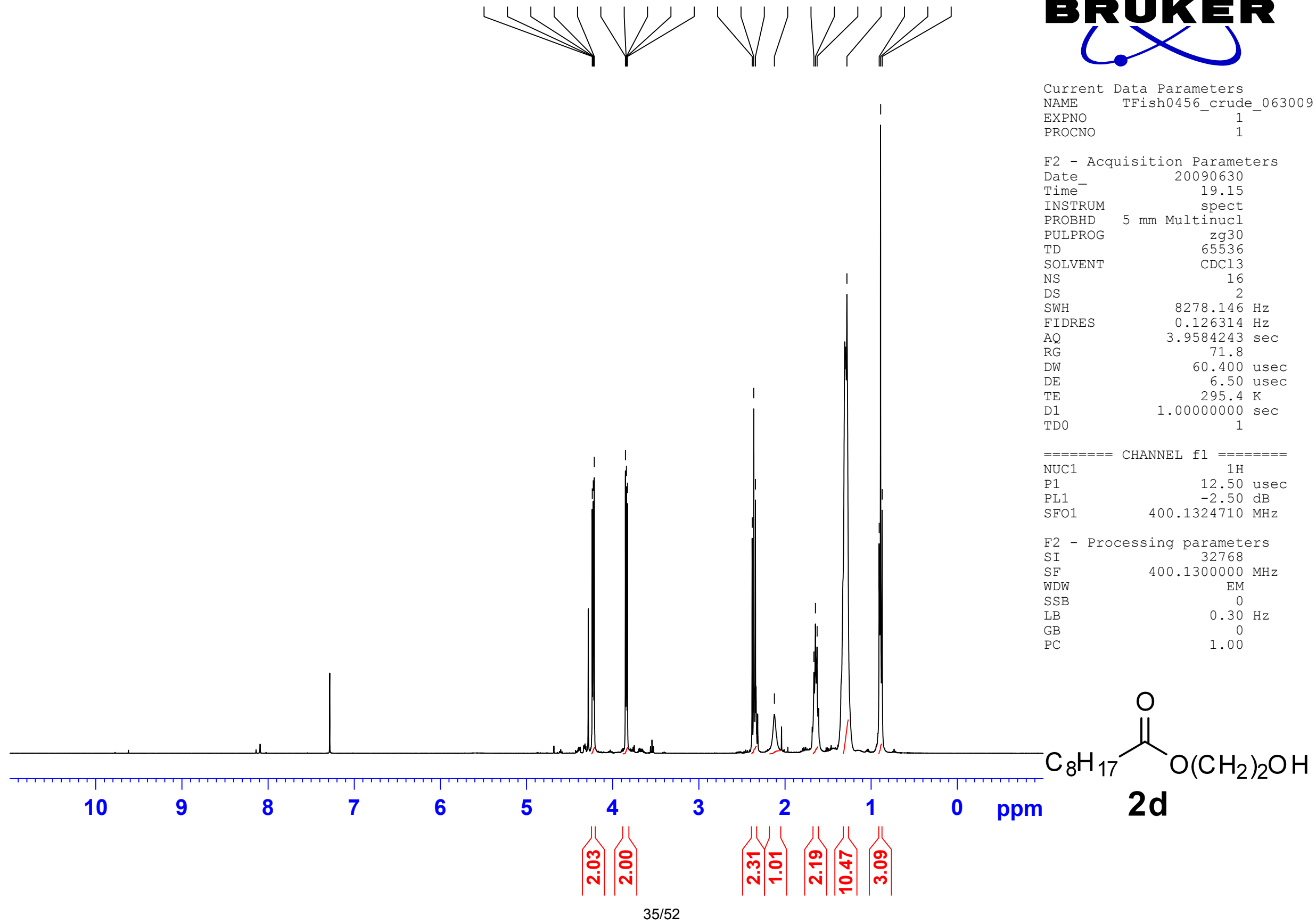

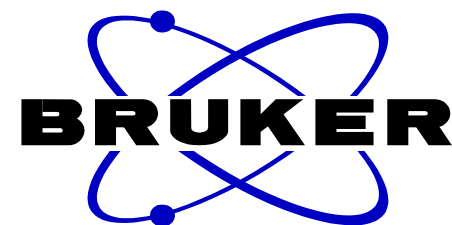

rent Data Parameters

NAME

- Acquisition Parameter

ime

OLVENT

DS

SWH

RG

$\mathrm{DE}$

D1 NUC1

PI

12.50 usec

F2 - Processing parameters

SI

$\mathrm{C}_{8} \mathrm{H}_{17} \overbrace{\mathrm{O}\left(\mathrm{CH}_{2}\right)_{2} \mathrm{OH}}$

2d 


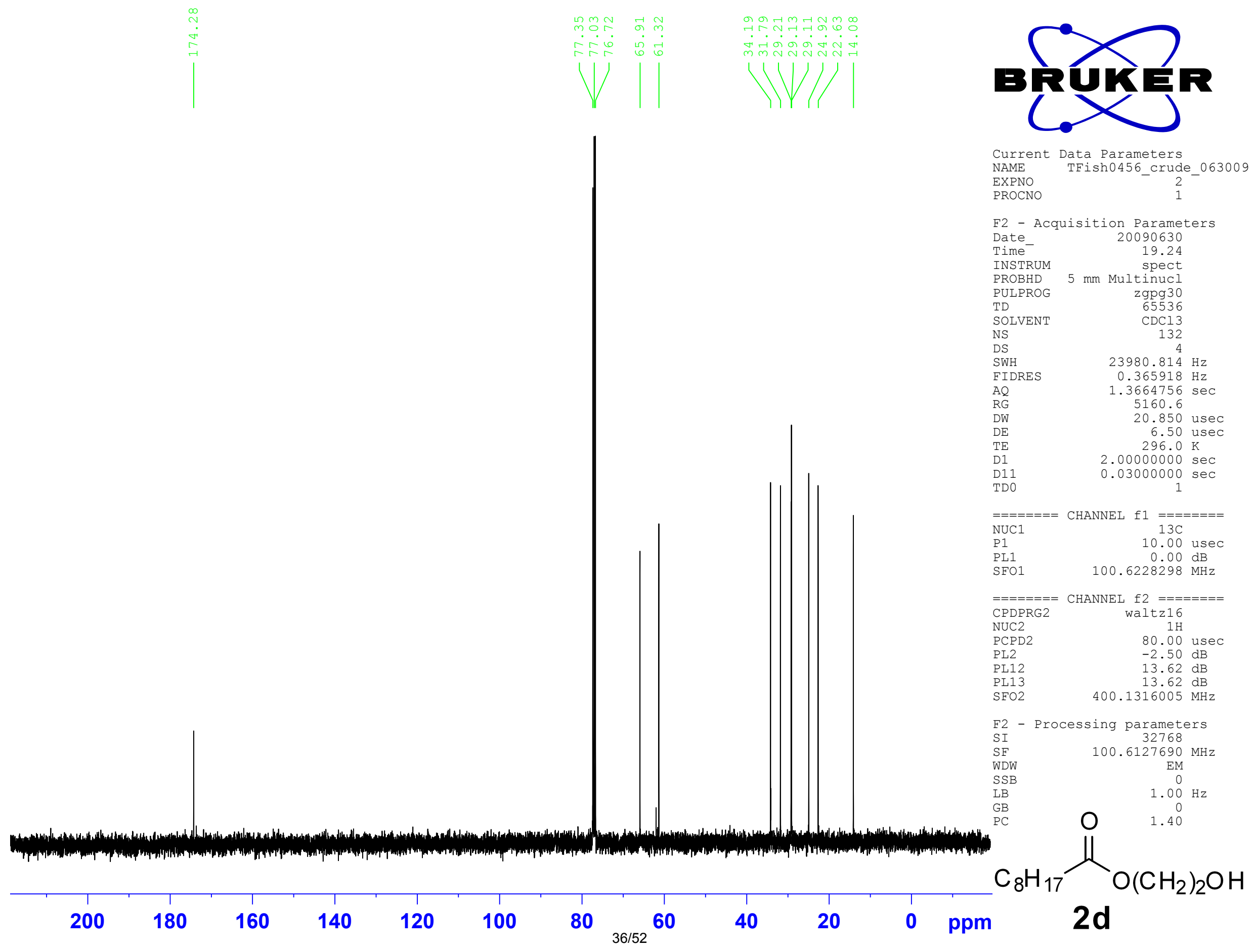




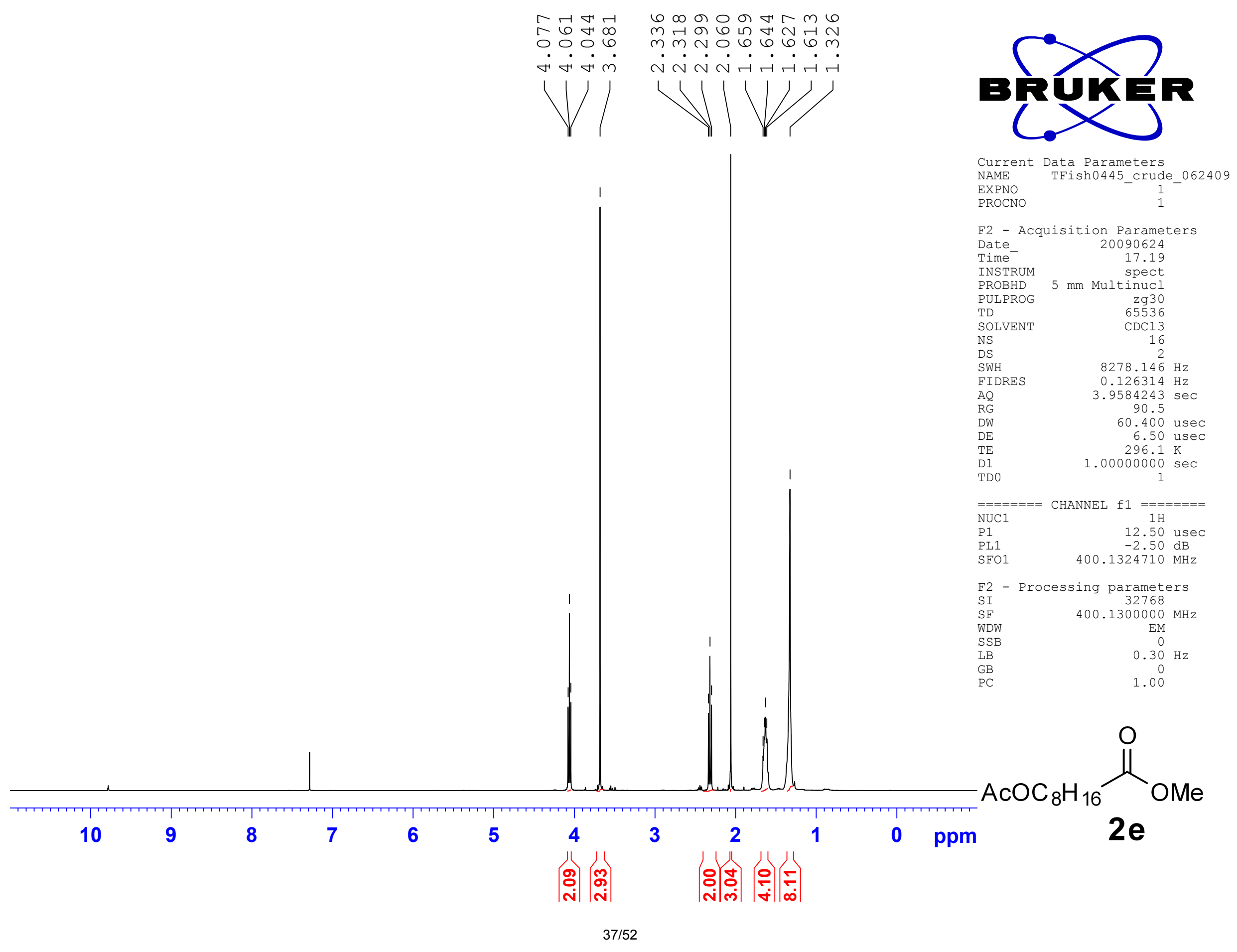




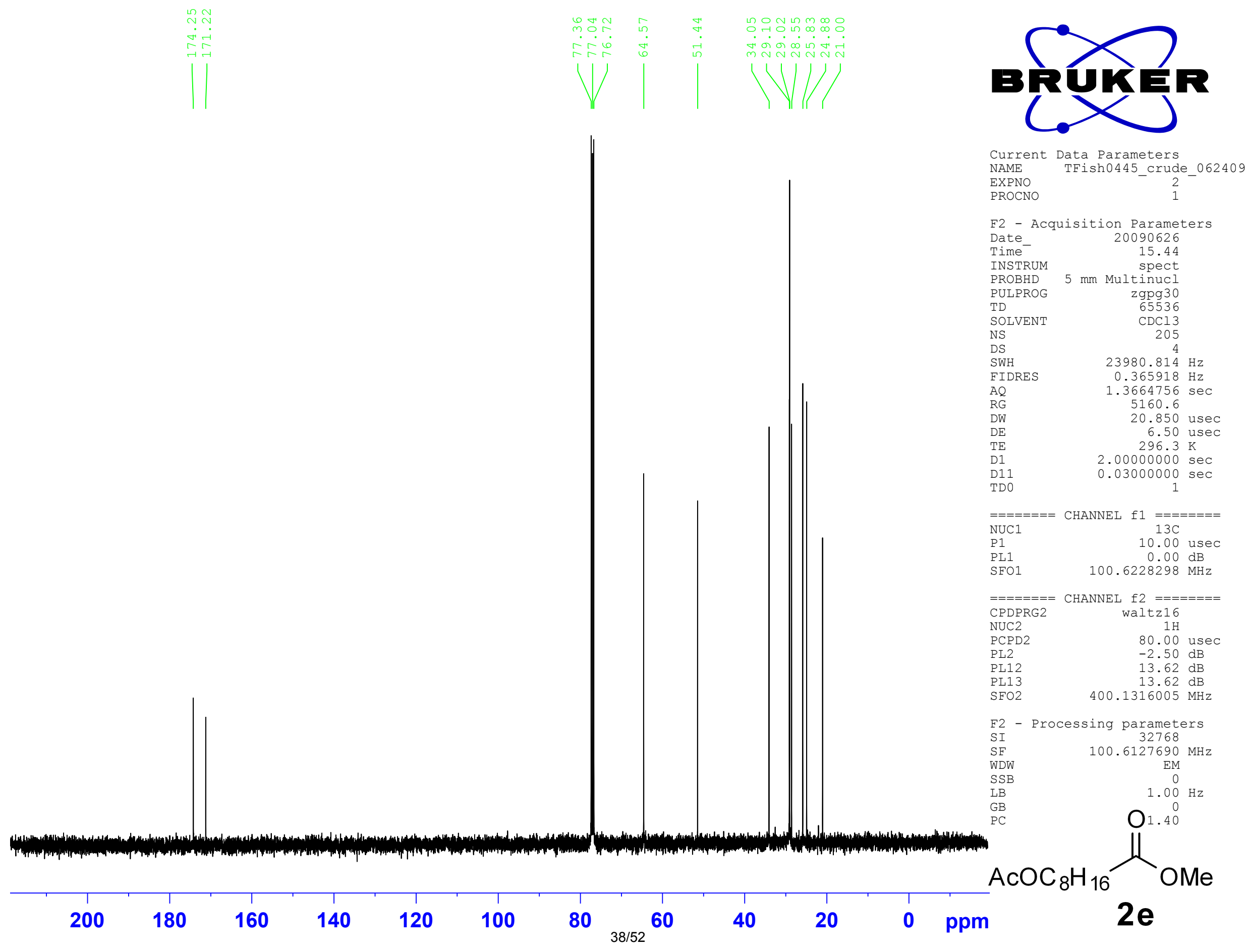




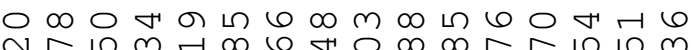

N

.

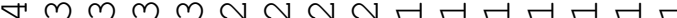
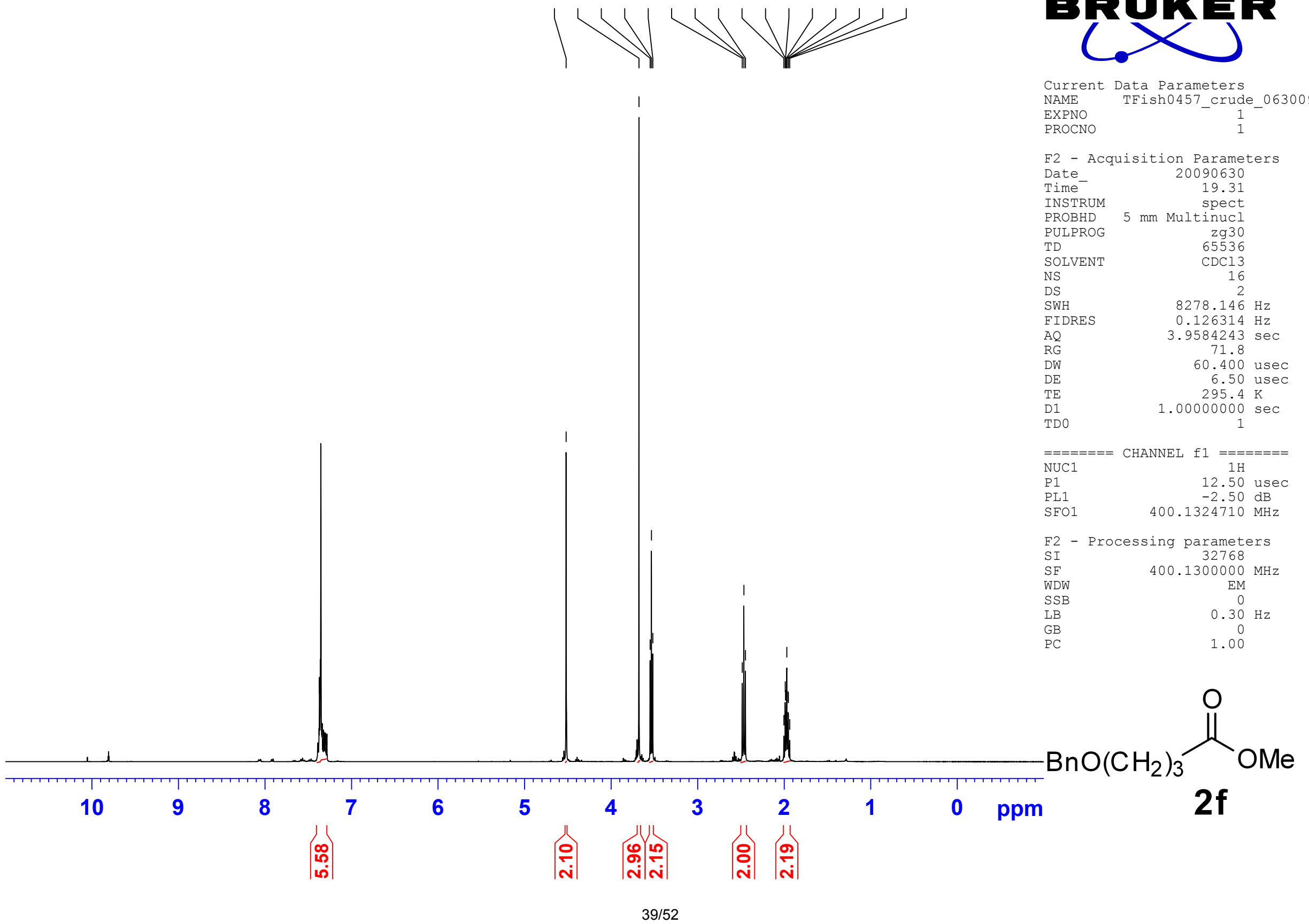

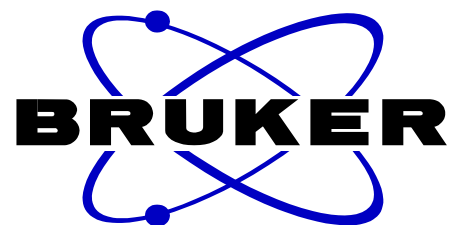

rent Data Parameter

NAME

- Acquisition Parameter

spect

SOLVEN

SWH

$\mathrm{DW}$

$\mathrm{E}$

TD 1

2 - Processing parameters

GB

$\mathrm{BnO}\left(\mathrm{CH}_{2}\right)_{3}$

\section{$2 f$}




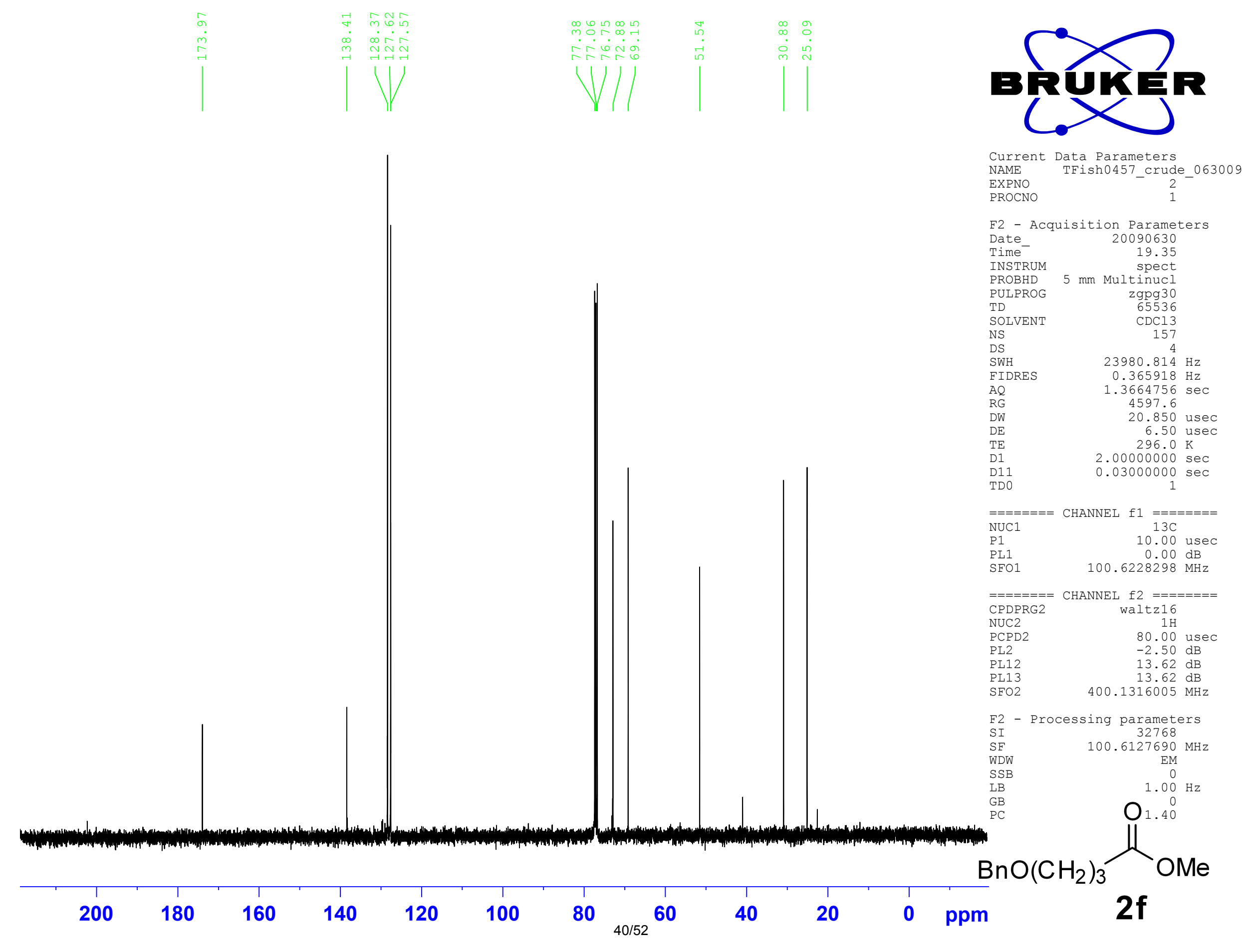


๓)

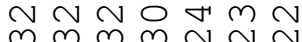

- . . . .

hrhen h

ull
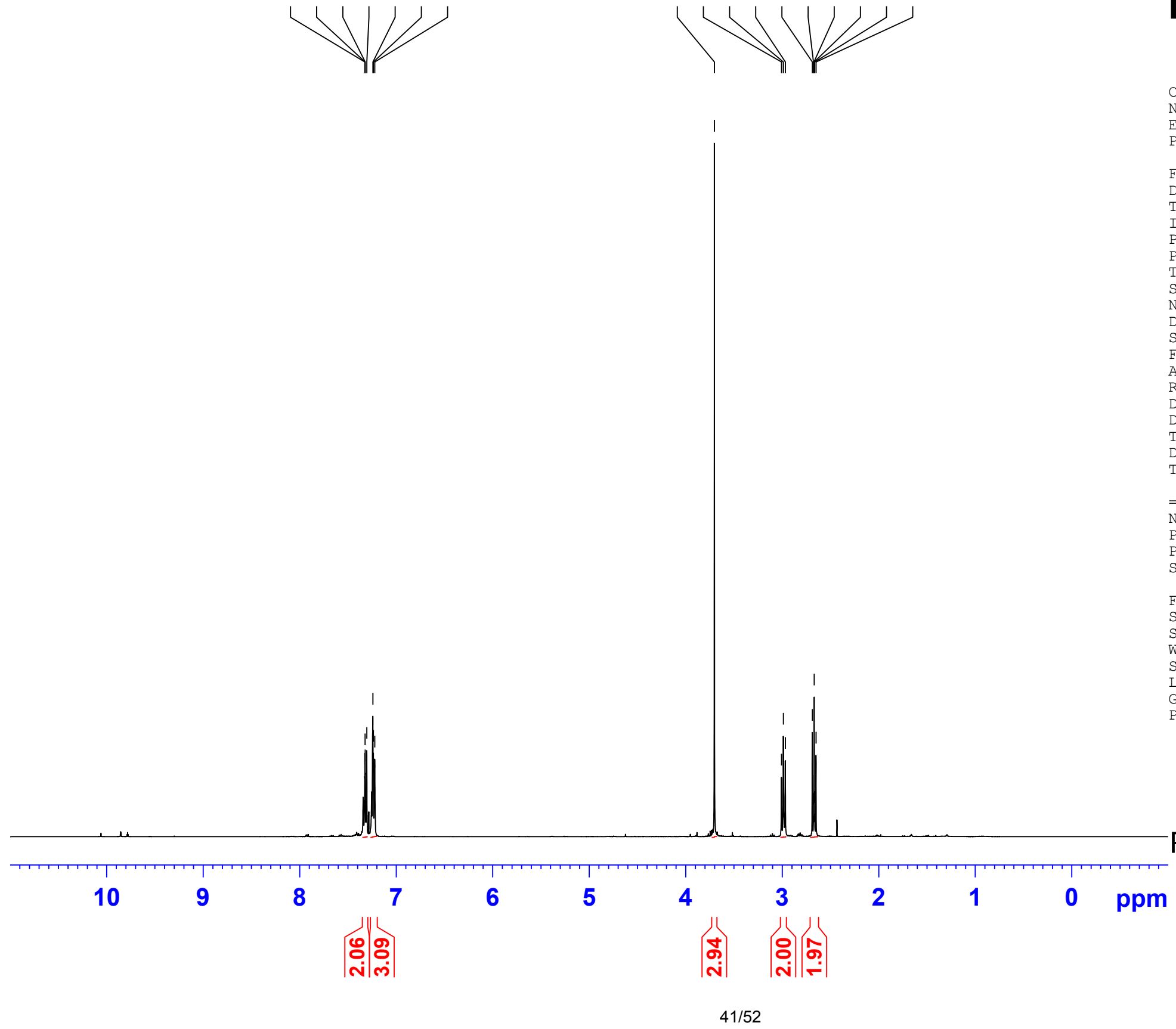

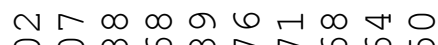
$\circ \circ$ ○ $\dot{m} \dot{m} \dot{\sim} \dot{\sim} \dot{\sim} \dot{\sim} \dot{\sim} \dot{\sim}$

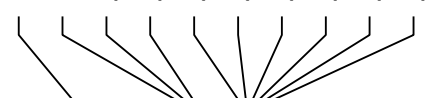

\section{$\mathrm{Ph}\left(\mathrm{CH}_{2}\right)_{2} \mathrm{OMe}$}<smiles>COC(=O)CCc1ccccc1</smiles>

$2 \mathrm{~g}$ 


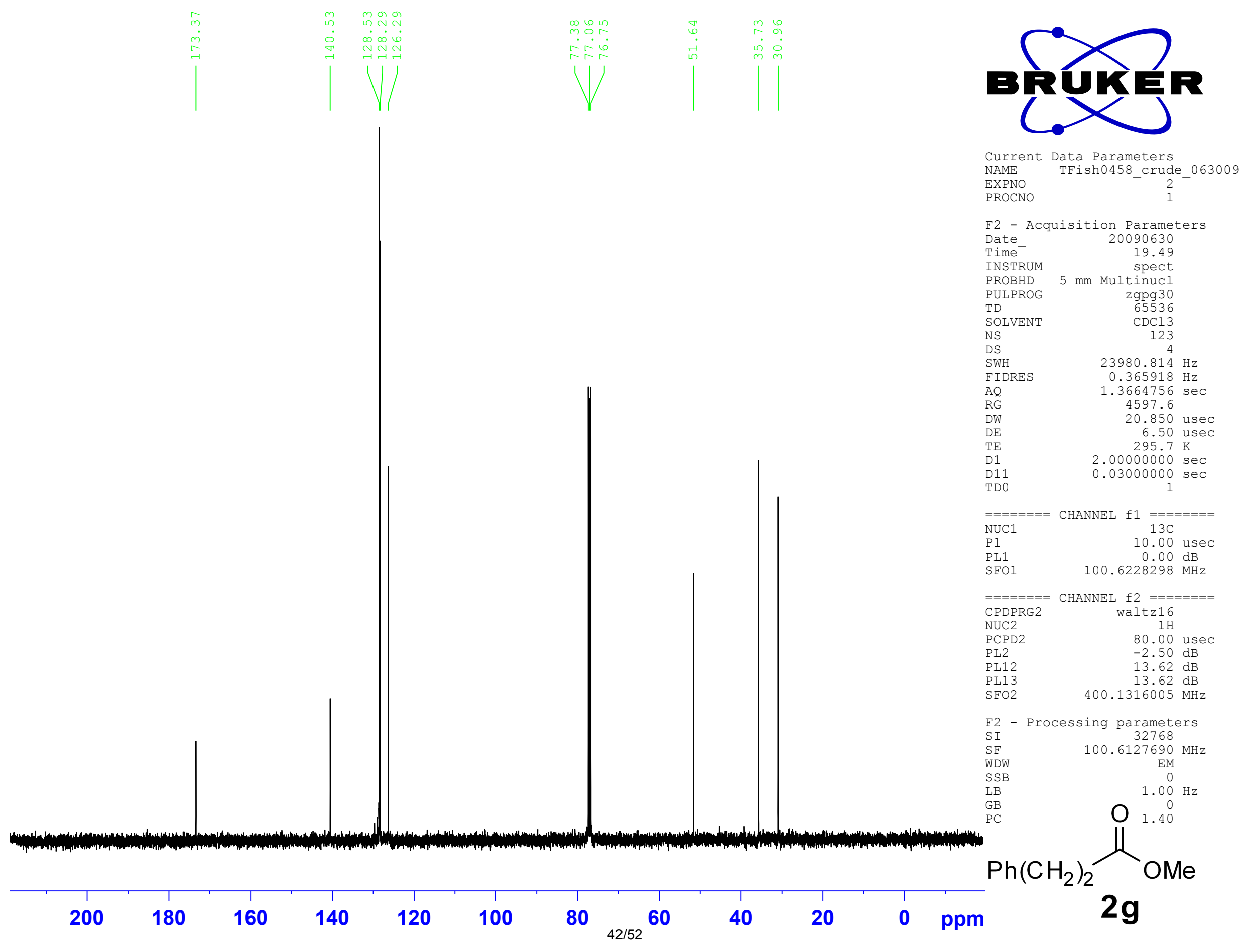




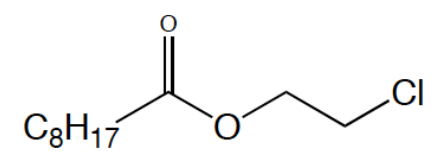

$2 \mathrm{~h}$

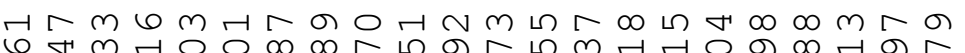
$m m m-1-6 m m m 6666 m m \sim \sim \sigma \infty$

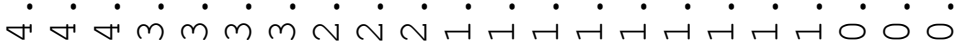
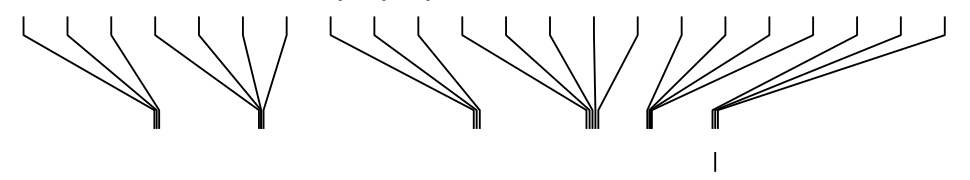

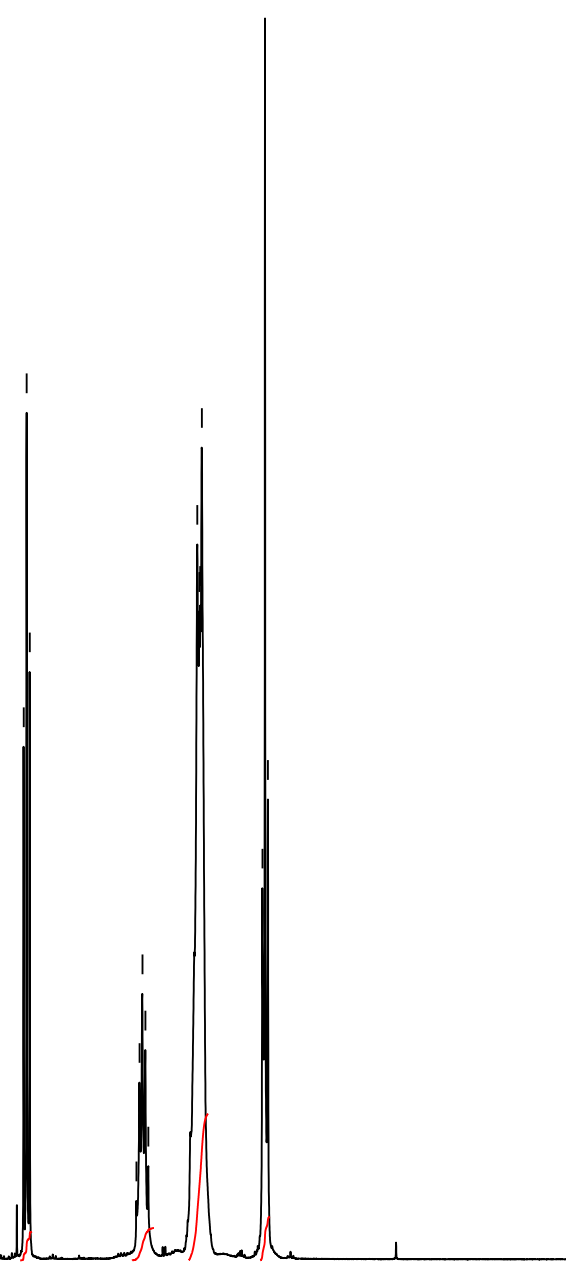

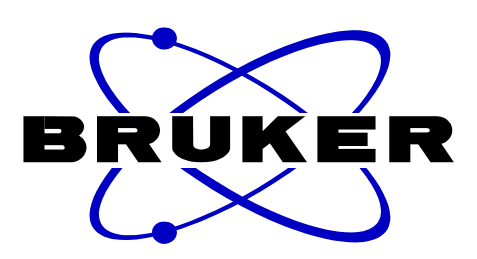

Current Data Parameters

NAME TFish0641_crude 061010

EXPNO

F2 - Acquisition Parameters

Date 20100603

$\begin{array}{ll}\text { Time } & - \\ \text { INSTRum } & 11.23\end{array}$

INSTRUM $5 \mathrm{spect}$

PULPROG 5 Z 30

TD

SOLVENT -65536

NS
DS
SWH

SWH

AQ

RG

DW

$\mathrm{DE}$

D1

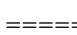

$\mathrm{NUC1}$

P1 1

PL1
SFO1

F2 - Processing parameters

SI 32768

SF $\quad 400.1300000 \mathrm{MHz}$

WDW

LB

GB
PC

EM

0
$0.30 \mathrm{~Hz}$

0
1.00

\section{0}

9

8

7

6

5

$\begin{array}{lll} & & \\ & & \\ \text { 인 } & & \\ & & \\ & & \\ & & \\ \text { N }\end{array}$

3

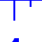

0 ppm

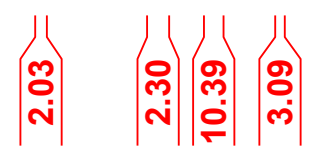




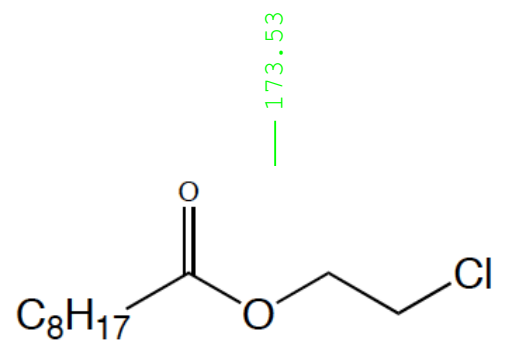

$2 \mathrm{~h}$

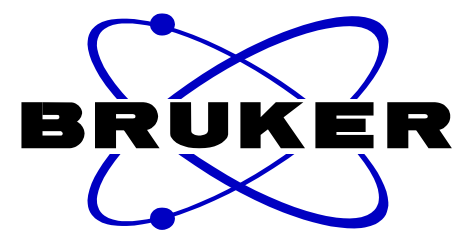

Current Data Parameters

NAME

Fish0641 crude 061010

PROCNO

F2 - Acquisition Parameters

Date 20100603

$\begin{array}{ll}\text { Time } & - \\ \text { INSTRUM } & 11.29\end{array}$

INSTRUM $5 \mathrm{~mm}$ SPect

PULPROG zgpg30

TD 65536

SOLVENT

NS

SWH

FIDRES

AQ

$\mathrm{RG}$
$\mathrm{DW}$
$\mathrm{DE}$

TE

D1
D11

TD0

$====$
NUC1
P1
PL1

$\mathrm{CDCl} 3$

153
4
$23980.814 \mathrm{~Hz}$
$0.365918 \mathrm{~Hz}$

$1.3664756 \mathrm{sec}$

32768

20.850 usec

6.50 use

$294.8 \mathrm{~K}$

$2.00000000 \mathrm{sec}$
$0.03000000 \mathrm{sec}$

CHANNEL f $13 \mathrm{C}$

10.00 usec $100.6228298 \mathrm{MHz}$

$=======$ CHANNEL $\mathrm{f} 2$

CPDPRG2 waltz16

TUC2

$\mathrm{PCPD} 2$

PL2 2

$\mathrm{PL} 12$
$\mathrm{PL} 13$

$\mathrm{PL} 13$
$\mathrm{SFO} 2$

$1 \mathrm{H}$
70.00

70.00 usec

$-3.35 \mathrm{~dB}$
$13.34 \mathrm{~dB}$

$13.34 \mathrm{~dB}$

$400.1316005 \mathrm{MHz}$

F2 - Processing parameters

$\begin{array}{ll}\text { SI } & 32768 \\ \text { SF } & 100.6127690 \mathrm{MHz}\end{array}$

WDW

LB

LB

GB 


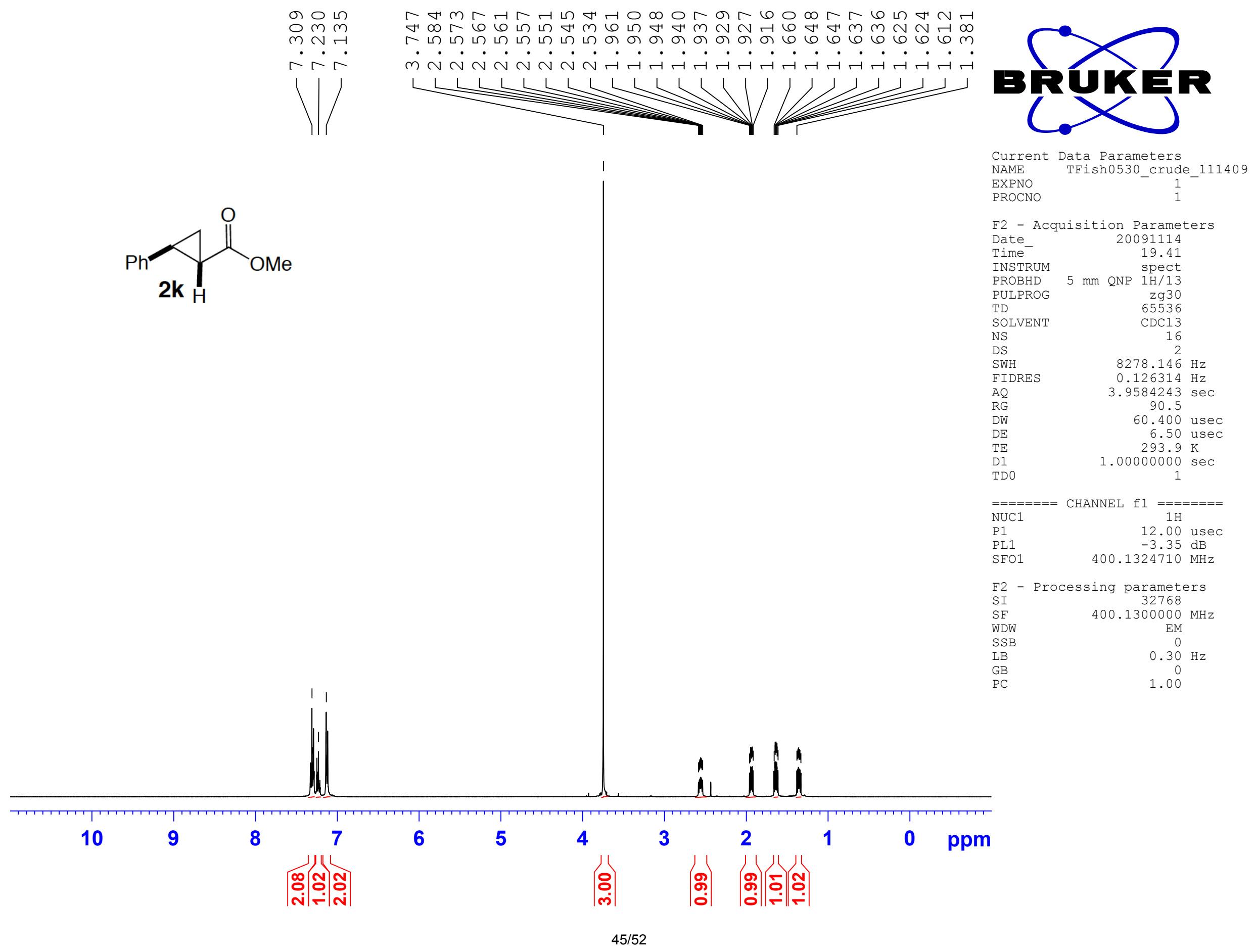




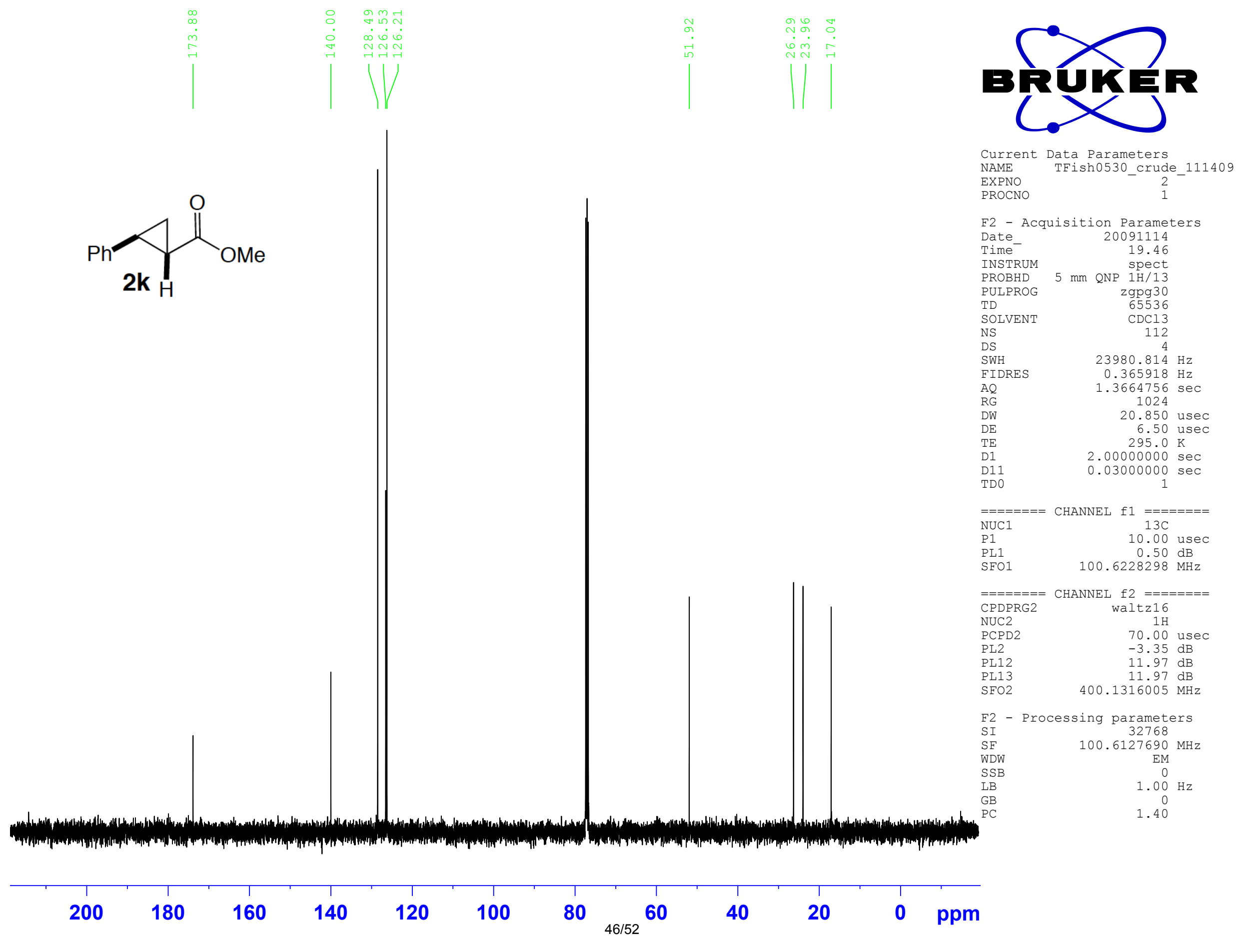




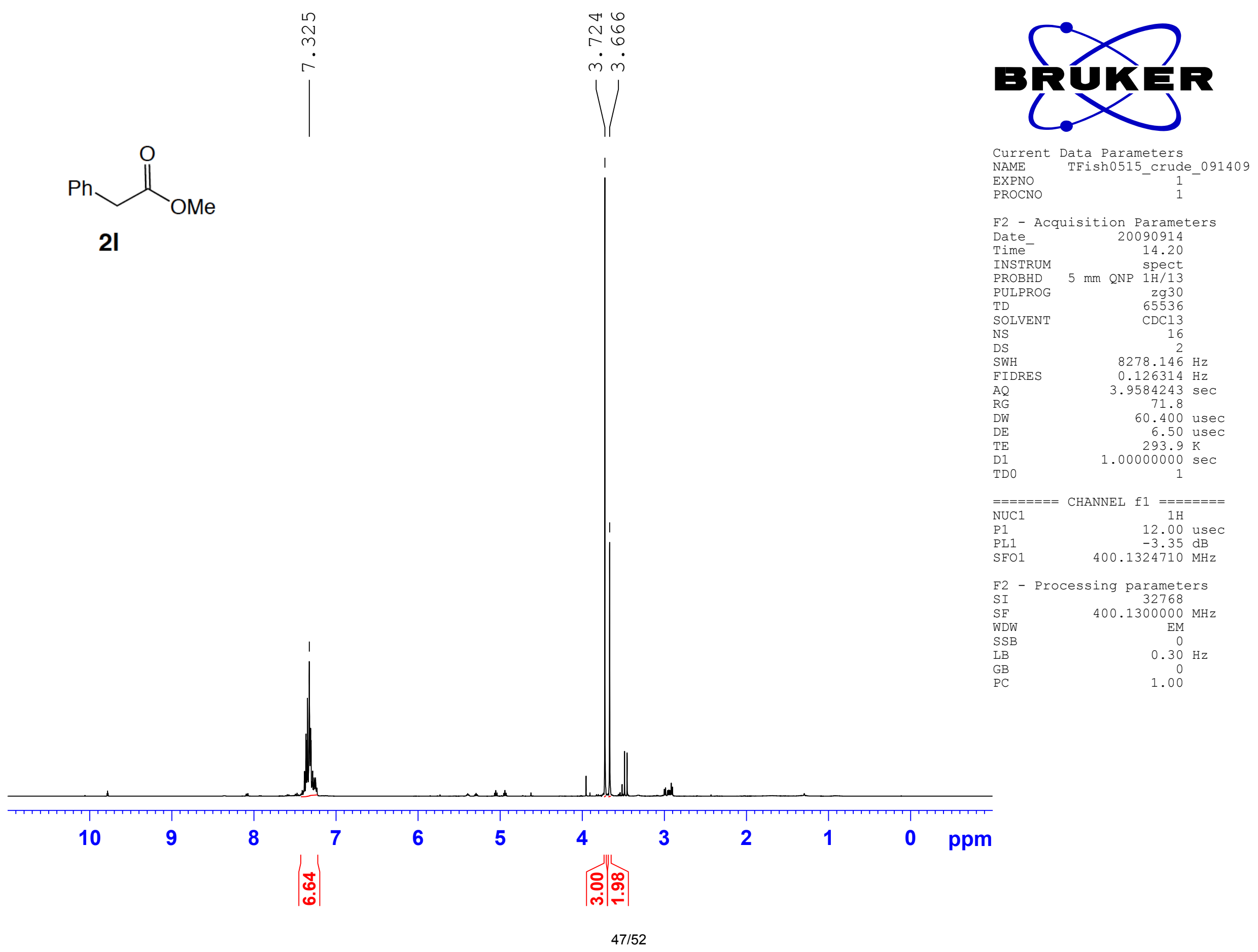




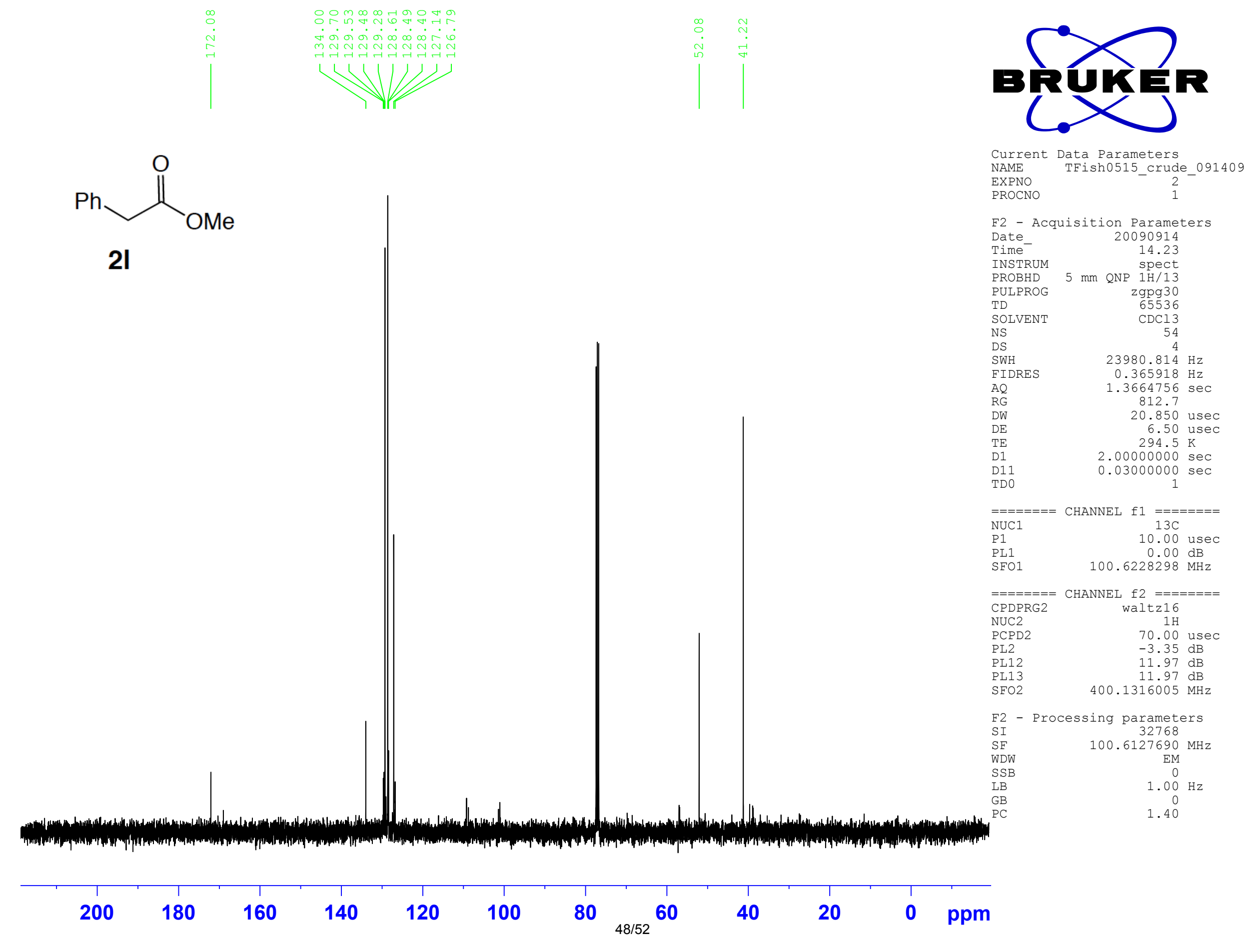




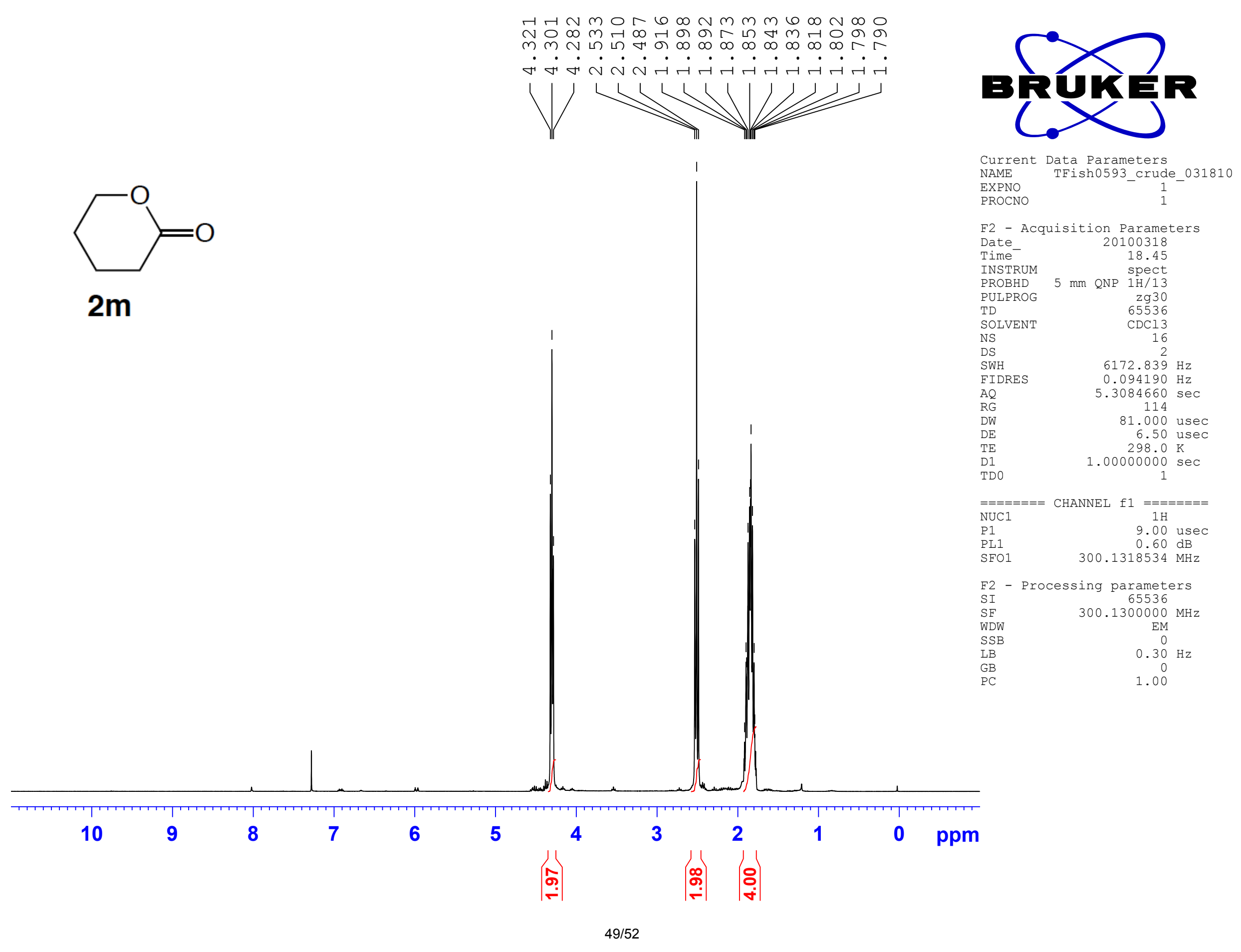




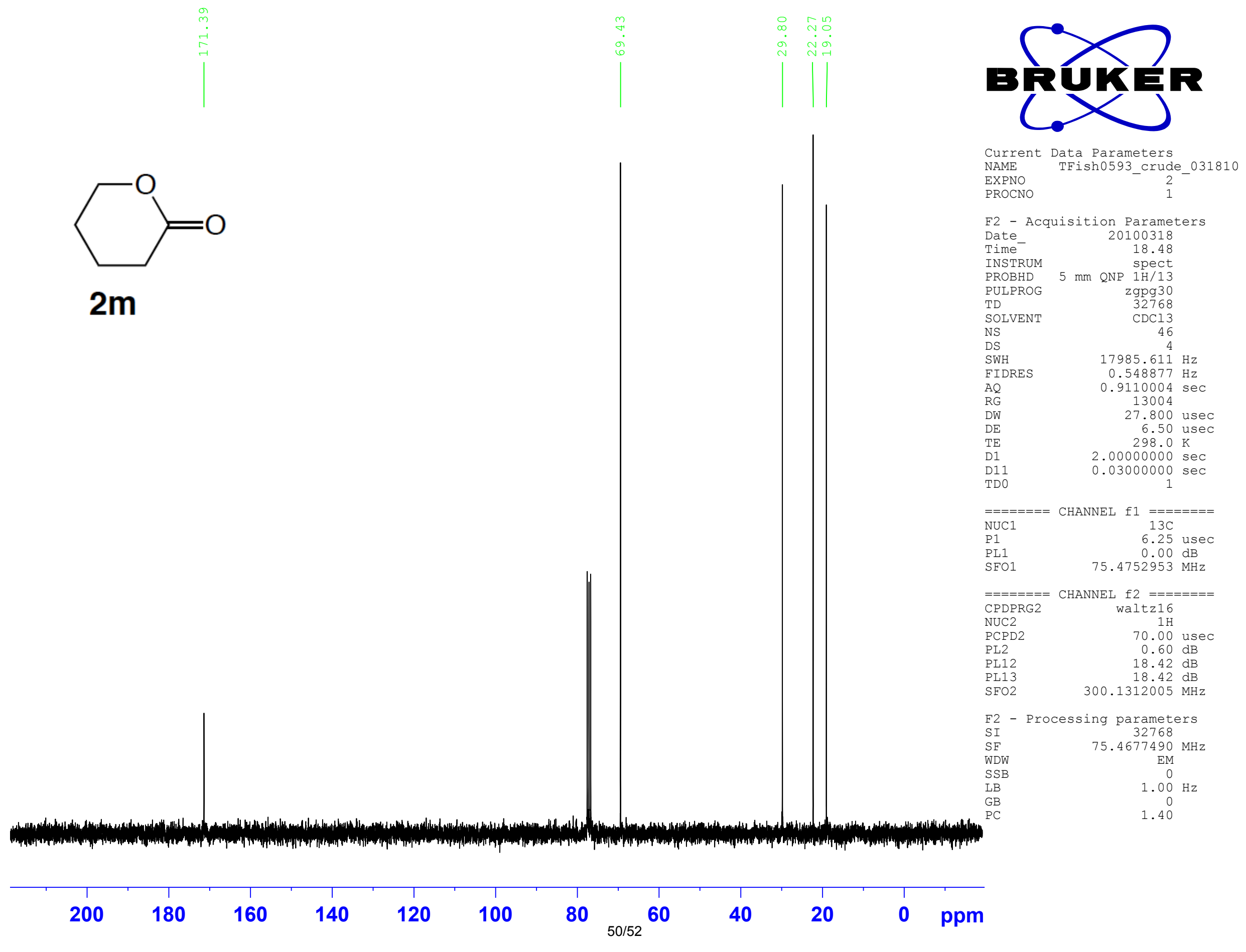




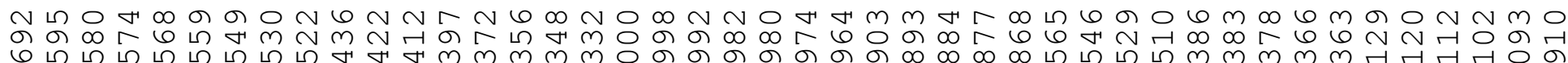

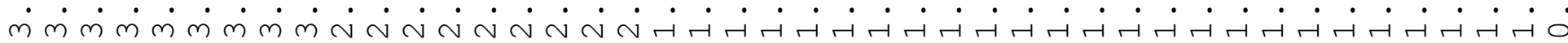

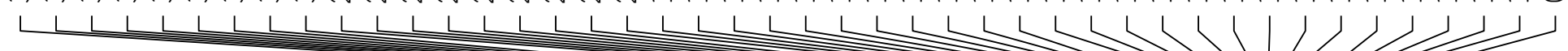<smiles>CC(=O)CCC(CCCl)CC(C)(C)C</smiles>

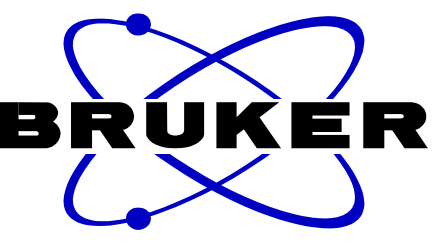

Current Data Parameters NAME TFish0589 10-17 031510 EXPNO PROCNO

F2 - Acquisition Parameters Date 20100315 Time 20.08 INSTRUM PROBHD $5 \mathrm{~mm}$ QNP $1 \mathrm{H} / 13$ PULPROG zg30 TD 65536 SOLVENT $\quad \mathrm{CDCl}$ DS SWH FIDRE

$\mathrm{AQ}$
$\mathrm{RG}$

$\mathrm{DW}$

DE

D1
TDO

\section{$=====$}

NUC

$\mathrm{P} 1$

$\mathrm{PL1} 1$
$\mathrm{SFO1}$

F2 - Processing parameters SI 32768 $400.1300000 \mathrm{MHz}$ WDW

400.1300000

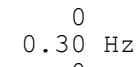

0.30
0

$8278.146 \mathrm{~Hz}$
$0.126314 \mathrm{~Hz}$ $3.9584243 \mathrm{sec}$

60.400 usec

6.50 usec

$1.00000000 \mathrm{sec}$

CHANNEL $\mathrm{f} 1$

$======$

10.25 usec

$-3.35 \mathrm{~dB}$
$400.1324710 \mathrm{MHz}$

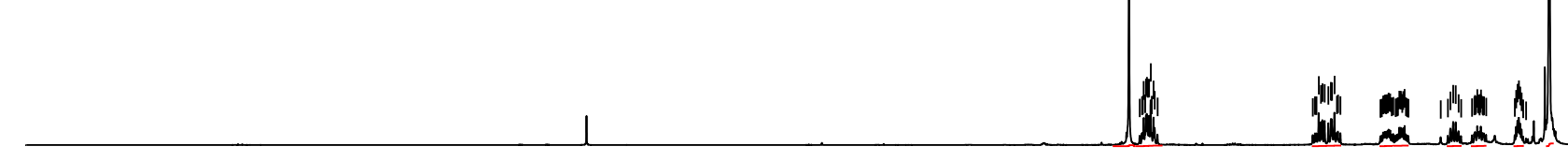

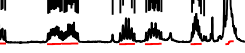

\begin{abstract}
8
\end{abstract}
7

6

5

4

$\left|\begin{array}{c|c}0 \\ 0 \\ m\end{array}\right| \begin{gathered}- \\ \text { in }\end{gathered} \mid$

3

1

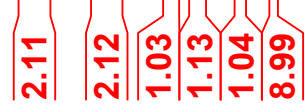

0 ppm 


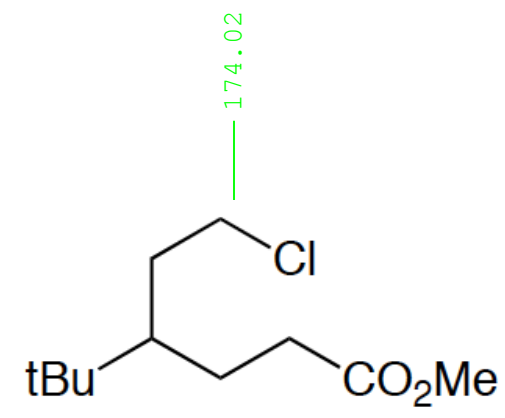

3

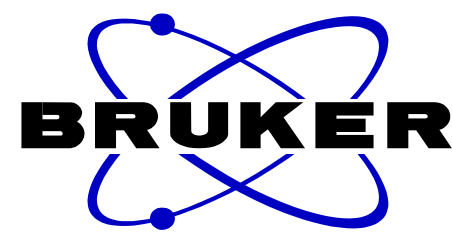

Current Data Parameters NAME

taish0589 10-17 031510 EXPNO

F2 - Acquisition Parameters Date 20100315 Time $^{-} \quad 20.13$ INSTRUM spect PROBHD $5 \mathrm{~mm}$ QNP 1H/13 $\begin{array}{lr}\text { PULPROG } & \text { zgpg30 } \\ \text { TD } & 65536\end{array}$

SOLVEN

NS

SWH

FIDRES

$\mathrm{AQ}$
$\mathrm{RG}$

DW

DE

D1
D11

D11
TD 0

$====$
NUC1
P1

$\mathrm{PL1} 1$
$\mathrm{SFO1}$

65536
$\mathrm{CDCl}$

58
4
23980.814

$23980.814 \mathrm{~Hz}$

$0.365918 \mathrm{~Hz}$

$1.3664756 \mathrm{sec}$

812 .

0.850 usec

6.50 use

298.0

$2.00000000 \mathrm{sec}$

$0.03000000 \mathrm{sec}$

CHANNEL $\mathrm{f}$ 1

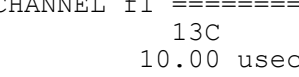

10.00 usec $100.6228298 \mathrm{MHz}$

$=======$ CHANNEL

CPDPRG

NUC2

PL2

PL12

PL13

WaNNEL $\mathrm{f} 2===$
waltz 16

70.00 usec

$-3.35 \mathrm{~dB}$

$13.34 \mathrm{~dB}$ $400.1316005 \mathrm{MHz}$

F2 - Processing parameters

$\begin{array}{ll}\text { SI } & 32768 \\ \text { SF } & 100.6127690 \mathrm{MHz}\end{array}$

WDW

SSB
LB
GB

GB

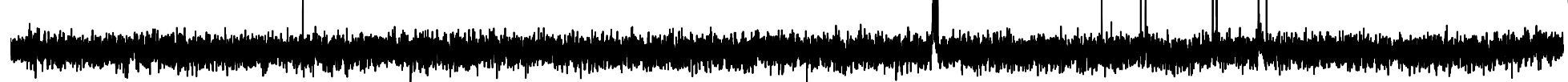

100.6127690
$\mathrm{EM}$

$1.00 \mathrm{~Hz}$

1.40 